

\section{ROLE OF SURFACE ROUGHNESS IN COLLOIDAL DYNAMICS OF DENSE SUSPENSIONS}

Beybin İlhan 



\title{
ROLE OF SURFACE ROUGHNESS IN COLLOIDAL DYNAMICS OF DENSE SUSPENSIONS
}

\author{
DISSERTATION
}

to obtain

the degree of doctor at the Universiteit Twente, on the authority of the rector magnificus, prof. dr. ir. A. Veldkamp, on account of the decision of the Doctorate Board to be publicly defended on Thursday 9 December 2021 at 12.45 hours

by

Beybin İlhan

born on the $20^{\text {th }}$ of October, 1988 in Ankara, Turkey 
This dissertation has been approved by:

Promoter:

Prof. dr. F.G. Mugele

Assistant Promoters:

Dr. M.H.G. Duits

Prof. dr. rer.-nat. S. Luding

Title: Role of surface roughness in colloidal dynamics of dense suspensions Author: Beybin İlhan

Cover design: Beybin İlhan

ISBN: 978-90-365-5308-7

DOI: $10.3990 / 1.9789036553087$

C 2021 Beybin Ilhan, The Netherlands. All rights reserved. No parts of this thesis may be reproduced, stored in a retrieval system or transmitted in any form or by any means without permission of the author. Alle rechten voorbehouden. Niets uit deze uitgave mag worden vermenigvuldigd, in enige vorm of op enige wijze, zonder voorafgaande schriftelijke toestemming van de auteur. 


\section{Graduation Committee:}

Chair: $\quad$ Prof. dr. J.L. Herek

Supervisor: $\quad$ Prof. dr. F.G. Mugele

Co- $\quad$ Dr. M.H.G. Duits

supervisors:

Prof. dr. rer.-nat. S. Luding

Committee Prof. dr. ir. J. Huskens

Members: $\quad$ Prof. dr. ir. W.M. de Vos

Dr. H.R. Vutukuri

Prof. dr. A. Van Blaaderen

Dr. D.J. Kraft

The research described in this thesis was performed at the Physics of Complex Fluids group within the MESA+ Institute for Nanotechnology and the Department of Science and Technology of the University of Twente. The research is financially supported by Netherlands Organization for Scientific Research (NWO-CW, ECHO grant 712.016.004 ). 

To my beloved family...

Aileme... 



\section{Contents}

\section{Chapter 1}

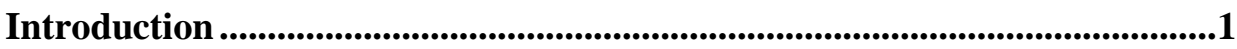

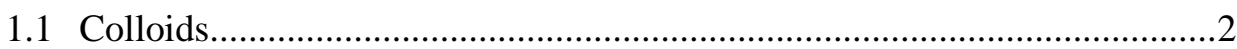

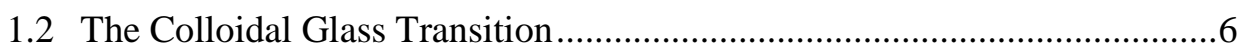

1.3 Real-time characterization of colloidal dynamics ......................................11

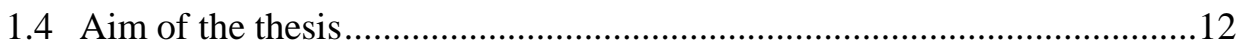

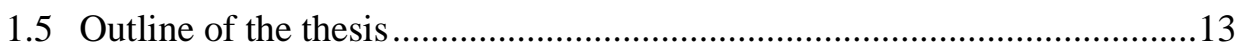

\section{Chapter 2}

\section{A Method for Reversible Control over Nano-roughness of Colloidal}

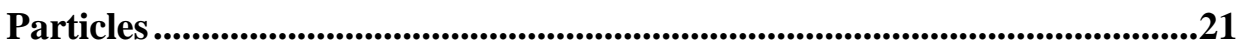

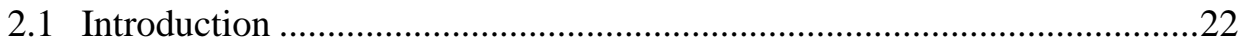

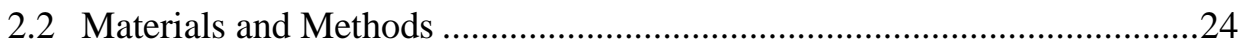

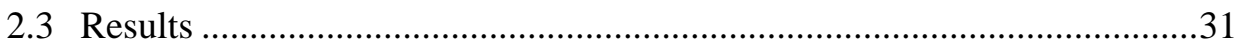

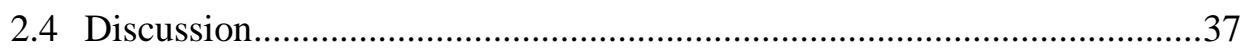

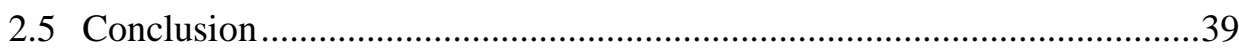

\section{Chapter 3}

Spherical Probes for Simultaneous Measurement of Rotational and Translational Diffusion in 3 Dimensions .............................................................51

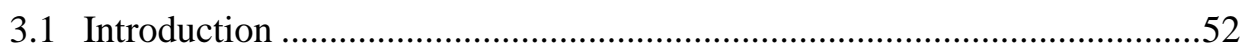

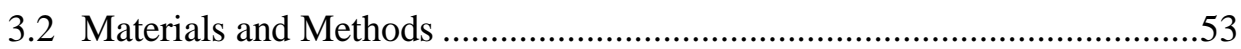

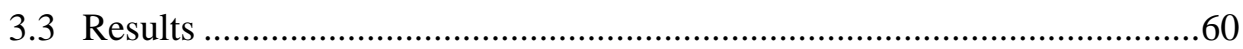

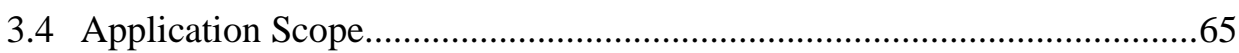

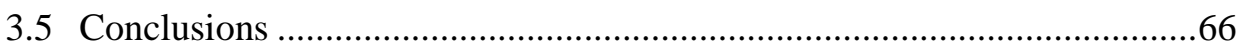

\section{Chapter 4}

Roughness Induced Rotational Slowdown near the Colloidal Glass

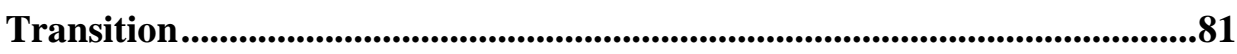

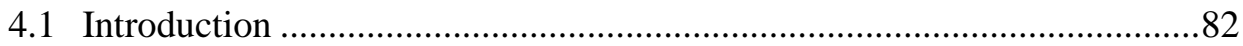

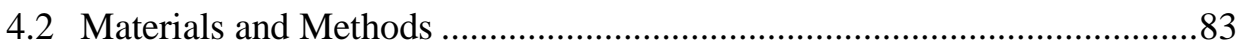

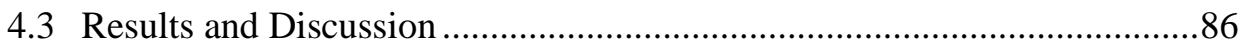

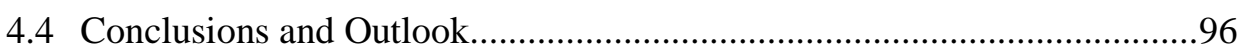




\section{Chapter 5}

Probing the Effect of Host Size on the Translational and Rotational

Dynamics of Rough Tracers .........................................................................109

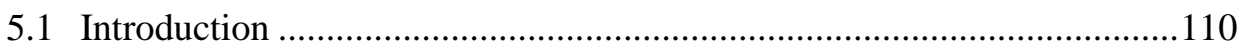

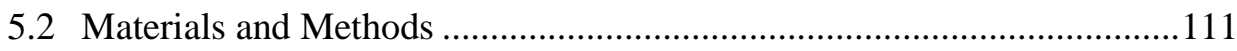

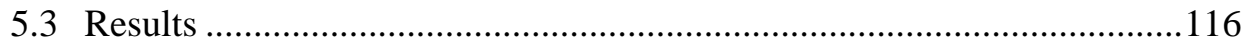

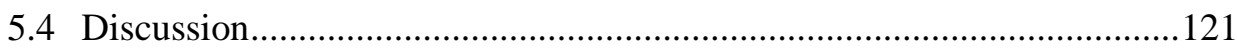

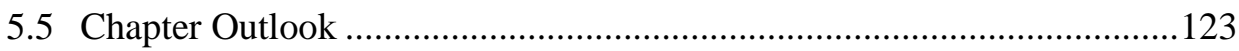

6 Summary ….................................................................................................129

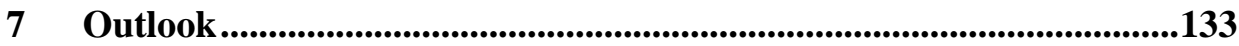

8 Samenvatting ............................................................................................137

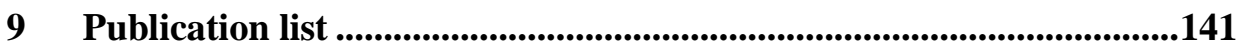

11 Acknowledgments.............................................................................................143 
Chapter 1

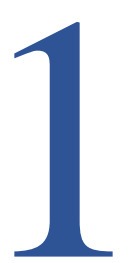

\section{Introduction}




\subsection{Colloids}

Colloidal systems are a class of soft materials consisting of particles that are 1 nanometer to 10 microns in size, dispersed in a continuous phase. The dispersed particles can be gas, liquid or solid. Based on the state of the dispersed constituents and the continuous phase, colloids can be categorized into different groups, such as solid particles in liquids (suspensions), liquid droplets in an immiscible liquid (emulsions), gas bubbles in solid or liquid (foams) and aerosols (liquid or solid particles in gas) [1]. The essential characteristic of the colloidal particles is their size. When dispersed, the particles are much larger than the continuum phase (solvent) molecules, and many molecules can interact with the particles via unrestrained collisions. Due to their small enough size, the particles exhibit Brownian motion due to thermal fluctuations arising from collisions. Analogous to molecules and atoms, the colloidal particles can move and rearrange spontaneously and exhibit different phase transitions. However, timescales for particle movement and phase transitions are much longer compared with atoms and molecules. Therefore colloids are often considered as "big atoms" [2] and have been widely used as model systems to understand many kinetic and equilibrium phenomena, such as phase transitions, glass transition and phase separations at experimentally accessible timescales [3, 4]. Another vital advantage of colloids related to their size is that the particles can be individually imaged via optical microscopy. Especially in systems with intrinsic heterogeneity (like glasses), linking the dynamics with the real-time locations can provide unique insights that cannot be obtained with techniques that only take an ensemble average over all particles [5,6]. In addition, colloids can be used as optical tracers to probe rheological properties on shorter length scales, such as properties of the microenvironments they are dispersed in (e.g., in foods, gels and emulsions) [7].

Recent years have seen significant developments in making colloids with complex architectures and functionalities. Examples include; ellipsoids [8, 9], colloidal bowls [10], cubes [11], dumbbells [12], colloidal molecules [13], polyhedral shaped particles [14], stars [15], tetrahedral shaped particles [16]. Given their wide variety in size, shape, material and tunability of their close-range interactions' strength and direction, colloids are excellent tools for understanding structure-function relationships. They can be used as building blocks for designing new materials with controllable properties [17]. For example, patchy particles with site-specific interactions are used as building blocks for designing 
functional materials [18-20]. Colloidal joints with flexible DNA linkers are shown to be excellent tools for designing soft robotics and reconfigurable materials with controllable motion range [21, 22]. Lock and key colloids which consist of spherical particles (keys) binding to the complementary particles with spherical cavity (locks) via depletion [23] or electric field [24], are considered as unique assemblies of flexible colloidal molecules.

Most applications of colloids in science, nature and industry involve particle volume fractions higher than $10 \%$. At such moderate or high concentrations, the interactions between the particles play an important role. Many studies have focused on how altering the particle characteristics, e.g., introducing shape and topographical irregularities, affects colloidal systems' collective behavior on the macroscopic level. It has been shown that introducing surface roughness and shape anisotropy generates or alters phenomena such as jamming, shear thickening and directed self-assembly [19, 25-29]. Understanding the dynamic interactions between such colloids and the contribution of geometric or topographical properties to the dynamics remains a standing quest with numerous unanswered questions. Several of these are: How do the dynamics of a dense colloidal system respond to an alteration in the surface topography of the particles? Can liquid to amorphous solid transition of colloidal suspensions be controlled via changes in topography? Can the motion of colloidal particles be restrained in different degrees of freedom when they are in close proximity? All of these questions and many more start with having a well-defined model rough colloidal system that can be designed and altered by demand. This thesis addresses these questions by providing an understanding of colloidal dynamics resulting from changes in surface characteristics. First, two synthetic methods to obtain model colloidal particles with specific topographical features are provided. The first presented method allows changing the topography of polymer latex particles in situ, and the second method offers design flexibility to synthesize and control the surface topography of spherical colloidal particles. Then colloidal dynamics of the latter model system are examined at conditions where particle-particle interactions are dominant.

From here on, to provide a fundamental level of understanding for readers of all levels, a brief introduction of colloidal interactions, colloidal diffusion and phase transitions are given. 


\subsubsection{The hard sphere model}

In a colloidal dispersion, particles can interact via attractive forces (such as the Van der Waals) and repulsive forces (such as electrostatic). The simplest model used in simulations and experiments for particle interactions is the hard sphere (HS) system [30, 31]. Hard Spheres comprise an ideal system of particles that do not experience any forces among themselves unless they are in contact. The particles have infinite repulsion when in contact; thus, they do not overlap. The HS pair potential is given by:

$$
\mathrm{U}_{\mathrm{HS}}(\mathrm{r})=\left\{\begin{array}{cc}
0, & \mathrm{r}>2 \mathrm{a} \\
\infty & \mathrm{r} \leq 2 \mathrm{a}
\end{array}\right.
$$

where a is the particle radius. In the experimental domain, these HS systems are typically made by suspending particles in a (near-) refractive index matching solvent to diminish the Van der Waals interactions, while repulsive interactions are made short-ranged by adding salt to screen the electrostatic forces (in polar solvents) or by coating particles with a sterically stabilizing short polymer brush (typically in non-polar solvents). For HS-like particles, the particle volume fraction $(\varphi)$ is the main control parameter for the phase behavior.

\subsubsection{Brownian Motion and Free Diffusion}

When HS colloidal particles are dispersed at low volume fractions $\varphi$ (typically a few percent or less), they effectively interact only with solvent molecules and undergo unrestrained collisions. The particles experience a randomly fluctuating force. Since this force does not have any preferred direction, the colloid's motion is a random walk. The average mean squared displacement (MSD) of the particle increases linearly over the elapsed time. The translational diffusion coefficient $\mathrm{D}_{\mathrm{T}}$ of a spherical particle dispersed in a Newtonian liquid is given by the StokesEinstein equation [32]:

$$
\mathrm{D}_{\mathrm{T}}=\frac{\mathrm{k}_{\mathrm{B}} \mathrm{T}}{6 \pi \eta \mathrm{a}}
$$

where $\mathrm{k}_{\mathrm{B}}$ is the Boltzmann constant, $\mathrm{T}$ is the temperature, $\mathrm{\eta}$ is the solvent viscosity, and a is the particle's radius. In steady-state systems, The MSD can be obtained as a time- and ensemble-average and the relation between diffusion coefficient (eq. 1.2) and the MSD is given by: 


$$
<\Delta \vec{r}(\tau)^{2}>=<[\vec{r}(t+\tau)-\vec{r}(t)]^{2}>=6 D_{T} \tau
$$

where $\Delta \overrightarrow{\mathrm{r}}$ is the displacement vector throughout $\tau$ (lag time) with initial time point $\mathrm{t}$, and the $<>$ indicates an averaging over all equivalent particles and lag times.

Like the translational motion, rotational Brownian motion is also a consequence of thermal fluctuations at low volume fractions. For spherical particles, the rotational diffusion coefficient, $D_{R}$, is given by Stokes-Einstein-Debye expression:

$$
D_{R}=\frac{k_{B} T}{8 \pi \eta a^{3}}
$$

Noting here that rotational Brownian motion has a larger dependence (inversely proportional to $\mathrm{a}^{3}$ ) on the particle size compared to translational motion. Akin to MSDs and eq. 1.3, mean squared angular displacement (MSAD) and the relation between $D_{R}$ and MSAD can be defined as:

$$
<\Delta \vec{\theta}(\tau)^{2}>=<[\vec{\theta}(\mathrm{t}+\tau)-\vec{\theta}(\mathrm{t})]^{2}>=4 \mathrm{D}_{\mathrm{R}} \tau
$$

\subsubsection{Colloidal phase transition}

One of the most remarkable behaviors exhibited by colloidal suspensions is that they can undergo spontaneous phase transitions, mimicking the phase transitions of atoms and molecules. As stated above, for HS colloids, the potential is zero when particles are not in direct contact. All the other possible particle configurations have zero potential energy, making the volume fraction $\varphi$ an essential control parameter for the phase behavior of colloidal systems. Figure 1.1 represents the phase diagram of suspensions of monodisperse hard spheres $[33,34]$. For $\varphi<0.49$, the HS system is in a fluid state. Upon gradually increasing $\varphi$, a coexistence state is reached, where the system can have both fluid and crystal domains. For a $\varphi$ range between 0.54 and 0.74 , crystalline structure occurs. The crystalline structure maximizes the available local volume to the free particles (coexisting with the crystal); thus, the system (as a whole) has higher configurational entropy than any disordered state (fluid and glass) [34]. The HS system can be packed at the maximum $\varphi$ of 0.74 with complete crystal packing. If the volume fraction is increased quickly enough to avoid crystal nucleation 
(between $0.49 \leq \varphi<0.58$ ), the HS colloidal system enters a supercooled regime. The glass transition occurs at $\varphi_{\mathrm{g}}=0.58$ for HS systems and persists till $\varphi=0.64$, which is called random close packing $\left(\varphi_{\mathrm{rcp}}\right)$ [31]. It should be noted here that, apart from the volume fraction being the control parameter for governing the phase transition in colloidal systems, another important element is the polydispersity of the suspensions. Often in experiments, to reach a glassy phase for HS systems, the suspension needs to have some level of polydispersity [33]. Colloidal suspensions with low polydispersity tend to form crystal structure, and for glassy systems, the borders of the $\varphi_{\mathrm{g}}$ for transitions and upper limit of the glassy regime $\left(\varphi_{\mathrm{rcp}}\right)$ depend on the polydispersity of the sample $[33,35]$.

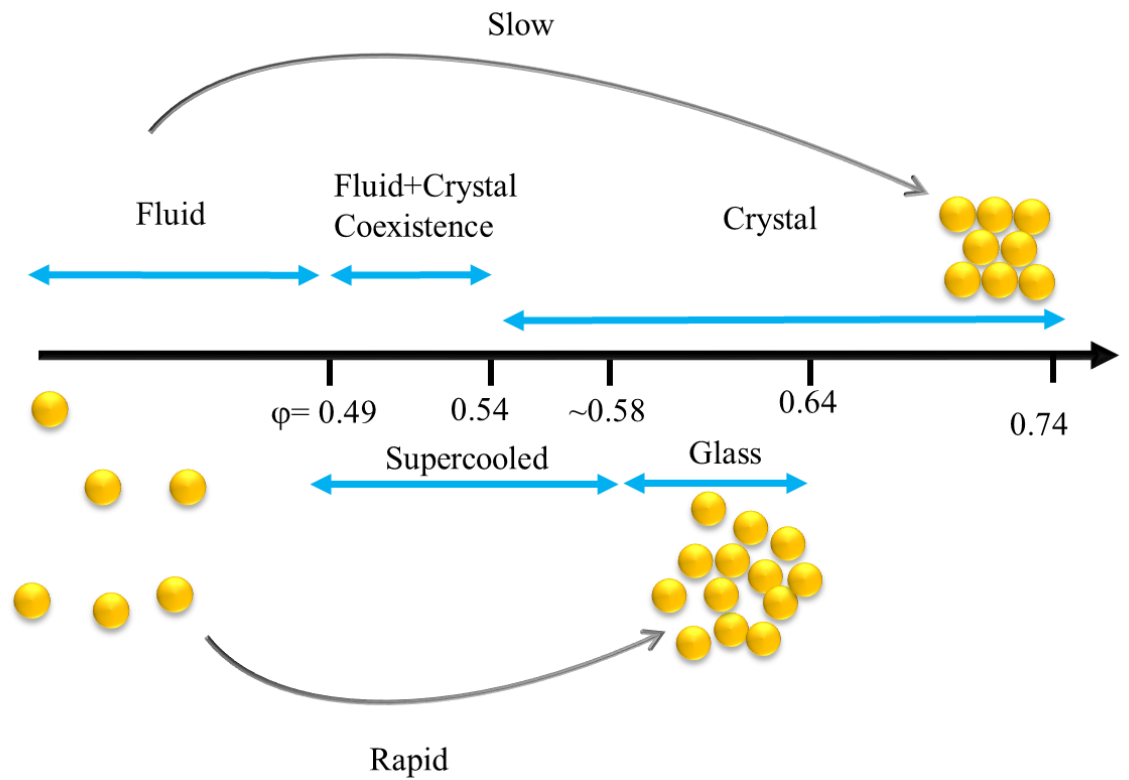

Figure 1.1. The phase diagram of colloidal hard spheres. Top and bottom arrows indicate that a transition from fluid to supercooled and then glassy state requires a rapid increase in $\varphi$ to avoid crystallization.

\subsection{The Colloidal Glass Transition}

Glasses are disordered solid materials that resemble crystalline solids due to their mechanical rigidity, but also resemble liquids due to their disordered molecular structure. These dual characteristics make understanding glass-forming materials 
and glass transition one of the most puzzling quests for scientists [36]. While the most common example of glasses is the window panes, the term "glass" can refer to a broad set of amorphous solids present in nature and technology, such as silicates, metals, polymers and biological cells [37, 38]. If a liquid is sufficiently and rapidly enough cooled below its melting point so that the cooling rate is faster than the crystal nucleation of the molecules, it can form an amorphous solid [39, 40]. During this rapid decrease in temperature, the material's viscosity (or relaxation time) increases by several orders of magnitude, and molecular motion slows down dramatically. From a practical perspective, this material is like a solid and cannot flow in an observable time scale.

Similar to molecular glasses, colloids can undergo glass transitions too. The first key experimental study of HS glass transition of colloidal systems was done by Pusey and van Megen with poly-methacrylate (PMMA) particles dispersed in a refractive index matching solvent [41]. Since then, colloids have been extensively used as model systems for glass transition studies [33, 34, 42]. Compared to their molecular and atomic analogs, the control parameter for glass transitions in colloidal systems is $\varphi$, rather than the temperature. At low volume fractions, the colloidal suspension is in the fluid state. Particles can diffuse and rearrange due to Brownian motion. If $\varphi$ is rapidly increased, the colloidal system can reach a supercooled and then glassy state [4, 43]. During this transition, particle dynamics slows down dramatically. The particles become confined by their neighbors and undergo substantial restrictions in their motions [4]. Any movement requires cooperative rearrangements of the surrounding particles [4446]. One remarkable property of supercooled liquids is the emergence of dynamic heterogeneity. Dynamic heterogeneity refers to that not all the relaxation process occurs uniformly within the material. Some regions may have faster dynamics than others, and any movement requires dynamic rearrangement of clusters.

While the transition from liquid to glass is widely seen, a unifying understanding of the underlying mechanism for the transition to the dynamically arrested glassy state does not exist $[36,47,48]$. This is because not all materials undergo glass transition in the same manner or rate. The viscosity growth with respect to the rapid quenching (either by decreasing the temperature or increasing $\varphi$ for colloids) and accompanying relaxation times can differ significantly from one system to another. 


\subsubsection{The Dynamic Slowdown}

Upon increasing the volume fraction, thus approaching the glass transition, the dramatic slowdown in dynamics occurs due to steric confinement of the particles by their surrounding neighbors. Particles become caged. As the lag time increases, this caged behavior shows its signature as MSDs with a plateau. Due to the cooperative motion of the particles at larger lag times, the MSD starts to increase and gains its diffusive signature (though with a reduction in diffusion coefficient) [49]. In contrast, on approaching the (translational) glass transition, the rotational Brownian motion of spherical particles still ensues only with a modest reduction in amplitude. A recent experimental study [50] showed that rotational Brownian motion exhibits almost diffusive behavior even close to the (translational) glass transition. Since spherical particles do not need to rearrange with their neighbors to rotate, they do not slow down due to steric confinement and do not undergo a rotational glass transition.

While the common hypothesis is that dynamical slowdown accompanying the glass transition results from a confinement effect in a broader sense, the level of confinement and the effective length scale governing the constraints in the translational and rotational degree of freedom are not universal for all systems. Studying the glass transitions in model spherical particles and their translational slowdown has provided significant contributions to our understanding of the glass transition [4, 49, 51-53], but the molecules and colloidal suspensions in nature and technology have non-trivial shapes and surface topography. Their diversity in shape and topography and resulting interactions at high concentrations can alter the interplay between translational and rotational motion and lead to diverse characteristics during glass formation. HS systems cannot solely model this behavior.

Building on recent developments in colloidal synthesis, many studies focus on the effect of shape anisotropy on the colloidal glass transition. Experiments with colloidal ellipsoids having different aspect ratios reveal two distinct glass transitions, translational and rotational. In recent studies, rotational freezing was observed to be followed by translational freezing at a higher onset volume fraction [54, 55]. One significant result from [54] is that the aspect ratio of the ellipsoids controls the onset and the separation between 2 glass transitions. The bigger the aspect ratio, the lower the onset of rotational glass transition and the larger the separation with the translational one. Another study investigated translational and rotational diffusion decoupling using different-sized dimer 
particles suspended in a generic glass-forming suspension of colloidal spheres [56]. It was found that at high volume fractions (i.e., near the glass transition), the long dimers' rotational motion slows down more than translations, whereas, for short dimers, the translational slowdown is more prominent than the rotational one. These differences in dynamic slowdown are attributed to the short dimers, being a close approximation of smooth spheres, able to rotate more freely; however, the long dimers' rotational motion is strongly hindered by caging due to neighboring host particles [56].

\subsubsection{Effect of roughness on the dynamical slowdown}

While approaching the glass transition, the confinement of the particles in cages can be considered the crucial aspect of the dynamic slowdown. As mentioned above, deviating from spherical symmetry via stretching from the spherical shape in a single direction (like ellipsoids) can alter the confinement effect, and particles can face different types of constraints in their translational and rotational degree of freedom. In its broad sense, the term "shape anisotropy" can refer to many different length scales for the particles. For example, colloidal molecules can be composed of many different blocks, and they can be formed by arranging clusters with various orders (such as tetrahedrons, pentamers, triangular dipyramids [57, 58]). The geometric details of a particle in a glass-forming system, such as the aspect ratio of the probe, size ratio of the surrounding neighbors, can significantly influence the caging network and dynamic slowdown (see Figure 1.2). This alteration in the caging network would result in different constraints in translational and rotational motion and confinement length scales. In this respect, considering only the shape anisotropy is not sufficient to reveal the microscopic underpinnings of the rotational and translational slowdown for all systems.

Considering the simple schematic drawn in Figure 1.2, smooth spherical particles are caged by their neighbors only translationally; they can still rotate in place without needing any cage-breaking event. For the sake of crude comparison, replacing the same spherical particles with similar-sized particles with outer geometry defined by decagons while keeping the surrounding neighbor network intact as the spherical particle case would introduce anisotropy in terms of geometric interaction length scales. While these particles are still translationally caged, the rotational motion would also be geometrically frustrated, i.e., particles would need to interact and rearrange themselves to allow rotational diffusion. The simplest translation of this geometric hindrance of rotational motion can be made by introducing alterations in surface geometry. The rotational motion of a 
colloidal system can also be constrained by introducing a different type of confinement introduced by surface asperities. Under conditions of close proximity of the particles, i.e., in dense suspensions, minor deviations from smooth topography can alter the interplay between translation and rotation. Due to their interacting surface asperities, particles can get spatially confined at different length scales for translations and rotations. Studying the rotational and translational slowdown with the presence of surface roughness would be a stepping stone for understanding the constraints in motions during the glass transition, and it can shed light on the effect of geometric hindrance in different degrees of freedom.
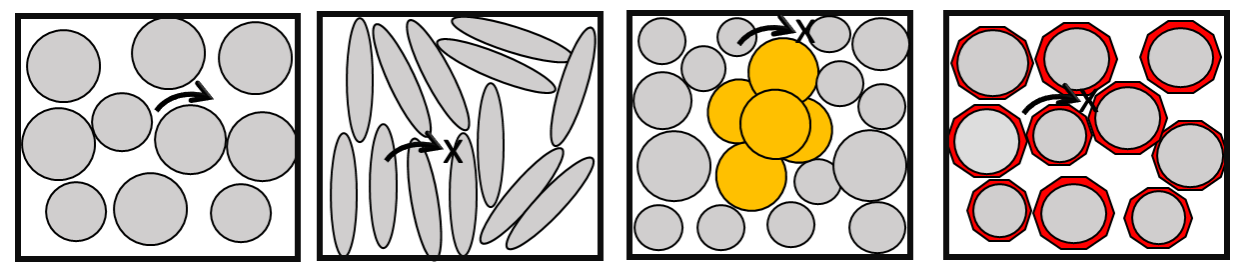

Figure 1.2. Schematic illustration of different particle systems at high concentrations, illustrating the translational and rotational mobility. From left to right: spherical particles, ellipsoidal particles, a colloidal molecule dispersed in a generic glass-forming system and spherical particles replaced with the surface replaced by decagons while keeping the neighboring network similar to the panel of spherical particles at the leftmost.

Also, from a macroscopic point of view, studying this uncharted territory would provide an opportunity to make a link between roughness-related microscopic dynamics and many rich macroscopic behaviors. A similar interplay of interactions due to surface roughness also applies in the presence of external stresses, e.g., shear. Particle surface roughness can lead to striking differences for macroscopic flow behavior and gravity settling of dense systems. It has been found that constraining rotational and sliding motion of the particles via frictional interactions among the particles (i.e., due to surface roughness) can lead to a significant increase in the dissipative energy of a dense suspension and a significant divergence in viscosity (discontinuous shear thickening, DST) at a critical shear rate $[25-27,59,60]$. Not only in the colloidal domain but also in granular systems, frictional properties can alter the jamming behavior and maximum packing fraction [61]. In this respect, understanding the effect of surface roughness in colloidal dynamics can create avenues to design the mechanical properties of suspensions. 


\subsection{Real-time characterization of colloidal dynamics}

As early as the discovery of the Brownian motion, microscopy has been used to study the dynamics of colloidal systems [62]. Real-time tracking of colloidal particles in three dimensions can shed light on many different phenomena in complex fluids' dynamics. Conventional optical microscopy is not well-suited for 3D imaging of dense colloidal systems, but confocal scanning laser microscopy (CSLM), combined with z-scanning, is a powerful tool. Confocal microscopy differs from conventional optical microscopes since out of focus light is vastly reduced using a pinhole aperture. Also, the use of fluorescence imaging is helpful since it allows to distinguish between different structural components in the sample. The principle is shown in Figure 1.3.a. Single-point illumination within the sample focal plane occurs through an illumination pinhole, depicted as blue lines, and the emitted fluorescent light (green lines) from the sample is collected through the objective and a dichroic mirror. The emitted light then reaches the detector pinhole, which is aligned with the image plane. In this way, out-of-focus light (dashed green lines) gets rejected. If the index of refraction of the colloids is matched with that of the dispersing medium, fluorescently labeled particles can be easily imaged deep within the sample, even for samples at high volume fractions. By combining the laterally scanned 2D images of focal planes at different heights, a 3D image stack can be obtained.

Following the image capture, several image processing steps are necessary to obtain time-dependent particle locations, which can be linked to structural and dynamic quantities of the entire sample. Particle center locations can be obtained within subpixel accuracy, using well-established detection algorithms [63, 64]. These locations can then be used to obtain information about the structure of the colloidal system. Time-dependent particle trajectories can be obtained by linking the positions at consecutive time points and can be further analyzed in terms of mean squared (angular) displacements to reveal colloidal dynamics. To illustrate the principle, Figure 1.3.b shows an example image of a low volume fraction system of fluorescently labeled polystyrene particles. Figure 1.3.c. illustrates the trajectories obtained from the 2D image series, where each trajectory is depicted with a different color. 
a)

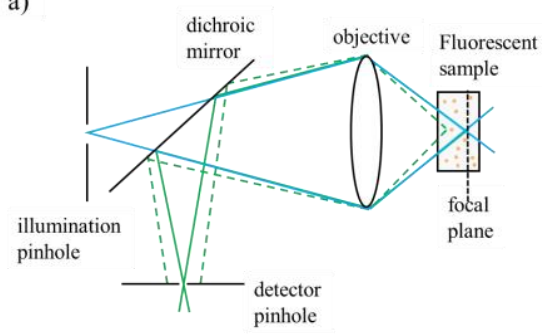

b)

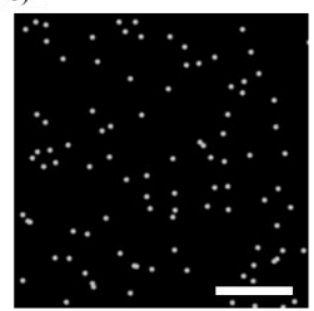

c)

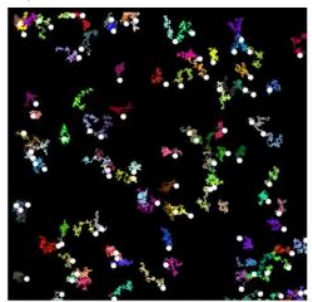

Figure 1.3. a) Schematic drawing of confocal microscopy (not drawn to scale). Dashed lines represent out of focus light, solid lines represent in focus light, b) Example confocal image of fluorescently labeled polystyrene particles (scale bar $=10 \mu \mathrm{m}$ ), c) Trajectories of the particles, obtained from the time dependence of images like panel $b$.

\subsection{Aim of the thesis}

Questions and present challenges outlined in the previous sections underline that a unifying understanding of the effect of surface topography, i.e., roughness, on the colloidal dynamics of dense suspensions is missing. Even though many present colloids in nature and technology consist of particles rough topography, the large plethora of the existing studies of dense suspensions focus on colloidal systems with smooth topography leaving the big question of "what is the role of surface roughness?" unanswered. One of the main reasons for this is that surface rough colloidal particles with explicit roughness characteristics are not easily accessible. In addition, resolving the rotational motion of particles with spherical symmetry is not trivial due to the lack of optical anisotropy. Disentangling the topography's role from the other colloidal properties, such as shape anisotropy and directionality of the interactions, starts with a model system with well characterized properties and accessing colloidal dynamics on a single particle level in terms of rotations and translations. In this thesis, two different methods to obtain surface rough particles are provided to address the absence of model systems. Then, one model system with well-defined topography characteristics and optical anisotropy is used to reveal the effect of roughness on colloidal dynamics near the glass transition. 


\subsection{Outline of the thesis}

This thesis addresses these questions and challenges in the following order.

Chapter 2 presents a novel and straightforward method for inducing and controlling nano-scale surface roughness on (water-based) polymer latex colloidal spheres by exposing them to the degassed ionic solutions. The presented method is fully reversible, meaning that the self-structured asperities on the colloids can be relaxed, and surface topography can be reversed back with mild thermal treatment without altering the surface chemistry.

Chapter 3 addresses the lack of optically anisotropic, spherical-shaped rotational probes in the literature. We provide a novel approach to synthesizing fluorescently labeled raspberry-like colloids with an optical anisotropy and give extensive details about accessing their translational and rotational motion simultaneously. The bottom-up synthetic control and the applicability of our approach are demonstrated via the results obtained with two types of probes, with different roughness amplitudes, dispersed in high and relatively low viscosity solvents. These raspberry-like rotational probes serve as model systems to study the effect of roughness on colloidal dynamics in the following chapters.

Chapter 4 provides a unifying insight into the dynamics of colloidal suspensions that emerge from a topography alteration to smooth spherical particles. Using the model rough system we introduced in Chapter 3 and time-resolved 3D confocal microscopy, we investigate the rotational and translational Brownian motion within a broad range of volume fractions. We identify two distinct glass transitions, translational and rotational, then provide a physical picture of this behavior by comparing the roughness length scale to interparticle distances and mapping the rotational dynamics onto the number of particle-particle contacts.

In Chapter 5, we translate and extend our study in Chapter 4 to other glass forming systems. We use the raspberry-like rough particles (from Chapter $\mathbf{3}$ ) as a tool to investigate the rotational and translational dynamics in a generic glass forming, host colloidal system with smooth surfaces. By varying the asperity/host size ratio, we investigate the rotational and translational dynamics as a function of host volume fraction. We show that the rotational slowdown is the strongest when the asperities and host particles are of equal size. We benchmark our results with a new type of rotational probes with an almost smooth surface topography 
(normal roughness amplitude < $1 \%$ radius). In the absence of asperities, these particles do not exhibit rotational arrest, and the rotational Brownian motion preserves a diffusive signature.

\section{Bibliography}

[1] J.K. Dhont, An introduction to dynamics of colloids, Elsevier1996.

[2] W. Poon, Colloids as big atoms, Science, 304 (2004) 830-831.

[3] W.K. Kegel, A. van Blaaderen, Direct observation of dynamical heterogeneities in colloidal hard-sphere suspensions, Science, 287 (2000) 290293.

[4] E.R. Weeks, J.C. Crocker, A.C. Levitt, A. Schofield, D.A. Weitz, Threedimensional direct imaging of structural relaxation near the colloidal glass transition, Science, 287 (2000) 627-631.

[5] Z. Cheng, T. Mason, Rotational diffusion microrheology, Physical review letters, 90 (2003) 018304.

[6] D. Anderson, D. Schaar, H.G.E. Hentschel, J. Hay, P. Habdas, E.R. Weeks, Local elastic response measured near the colloidal glass transition, Journal of Chemical Physics, 138 (2013).

[7] T. Moschakis, Microrheology and particle tracking in food gels and emulsions, Current Opinion in Colloid \& Interface Science, 18 (2013) 311-323.

[8] M. Voggenreiter, J.r. Roller, J. Geiger, L. Ebner, A. Zumbusch, J.-M. Meijer, Preparation and Tracking of Oblate Core-Shell Polymethyl-Methacrylate Ellipsoids, Langmuir, 36 (2020) 13087-13095.

[9] S. Sacanna, L. Rossi, B. Kuipers, A. Philipse, Fluorescent monodisperse silica ellipsoids for optical rotational diffusion studies, Langmuir, 22 (2006) 1822-1827.

[10] J.M. Meijer, J.J. Crassous, Phase Behavior of Bowl-Shaped Colloids: Order and Dynamics in Plastic Crystals and Glasses, Small, 14 (2018) 1802049. 
[11] L. Rossi, S. Sacanna, W.T. Irvine, P.M. Chaikin, D.J. Pine, A.P. Philipse, Cubic crystals from cubic colloids, Soft Matter, 7 (2011) 4139-4142.

[12] B. Peng, H.R. Vutukuri, A. van Blaaderen, A. Imhof, Synthesis of fluorescent monodisperse non-spherical dumbbell-like model colloids, Journal of Materials Chemistry, 22 (2012) 21893-21900.

[13] D.J. Kraft, W.S. Vlug, C.M. van Kats, A. van Blaaderen, A. Imhof, W.K. Kegel, Self-assembly of colloids with liquid protrusions, Journal of the American Chemical Society, 131 (2009) 1182-1186.

[14] H.R. Vutukuri, A. Imhof, A. Van Blaaderen, Fabrication of Polyhedral Particles from Spherical Colloids and Their Self-Assembly into Rotator Phases, Angewandte Chemie International Edition, 53 (2014) 13830-13834.

[15] S.-M. Lee, Y.-w. Jun, S.-N. Cho, J. Cheon, Single-crystalline star-shaped nanocrystals and their evolution: programming the geometry of nano-building blocks, Journal of the American Chemical Society, 124 (2002) 11244-11245.

[16] E.C. Greyson, J.E. Barton, T.W. Odom, Tetrahedral Zinc Blende Tin Sulfide Nano-and Microcrystals, small, 2 (2006) 368-371.

[17] A. van Blaaderen, Colloids get complex, Nature, 439 (2006) 545-546.

[18] D.J. Kraft, J. Hilhorst, M.A. Heinen, M.J. Hoogenraad, B. Luigjes, W.K. Kegel, Patchy polymer colloids with tunable anisotropy dimensions, The Journal of Physical Chemistry B, 115 (2011) 7175-7181.

[19] D.J. Kraft, R. Ni, F. Smallenburg, M. Hermes, K. Yoon, D.A. Weitz, A. van Blaaderen, J. Groenewold, M. Dijkstra, W.K. Kegel, Surface roughness directed self-assembly of patchy particles into colloidal micelles, Proceedings of the National Academy of Sciences, 109 (2012) 10787-10792.

[20] S. Glotzer, M. Solomon, N.A. Kotov, Self-assembly: From nanoscale to microscale colloids, (2004).

[21] I. Chakraborty, V. Meester, C. van der Wel, D.J. Kraft, Colloidal joints with designed motion range and tunable joint flexibility, Nanoscale, 9 (2017) 78147821. 
[22] R.W. Verweij, P.G. Moerman, N.E. Ligthart, L.P. Huijnen, J. Groenewold, W.K. Kegel, A. van Blaaderen, D.J. Kraft, Flexibility-induced effects in the Brownian motion of colloidal trimers, Physical Review Research, 2 (2020) 033136.

[23] S. Sacanna, W.T. Irvine, P.M. Chaikin, D.J. Pine, Lock and key colloids, Nature, 464 (2010) 575-578.

[24] M. Kamp, N.A. Elbers, T. Troppenz, A. Imhof, M. Dijkstra, R. Van Roij, A. Van Blaaderen, Electric-field-induced lock-and-key interactions between colloidal spheres and bowls, Chemistry of Materials, 28 (2016) 1040-1048.

[25] L.C. Hsiao, S. Jamali, E. Glynos, P.F. Green, R.G. Larson, M.J. Solomon, Rheological State Diagrams for Rough Colloids in Shear Flow, Physical Review Letters, 119 (2017).

[26] C.P. Hsu, S.N. Ramakrishna, M. Zanini, N.D. Spencer, L. Isa, Roughnessdependent tribology effects on discontinuous shear thickening, Proceedings of the National Academy of Sciences of the United States of America, 115 (2018) 5117-5122.

[27] A. Singh, C. Ness, R. Seto, J.J. de Pablo, H.M. Jaeger, Shear Thickening and Jamming of Dense Suspensions: The "Roll" of Friction, Physical Review Letters, 124 (2020).

[28] E. Blanco, D.J. Hodgson, M. Hermes, R. Besseling, G.L. Hunter, P.M. Chaikin, M.E. Cates, I. Van Damme, W.C. Poon, Conching chocolate is a prototypical transition from frictionally jammed solid to flowable suspension with maximal solid content, Proceedings of the National Academy of Sciences, 116 (2019) 10303-10308.

[29] A. Donev, I. Cisse, D. Sachs, E.A. Variano, F.H. Stillinger, R. Connelly, S. Torquato, P.M. Chaikin, Improving the density of jammed disordered packings using ellipsoids, Science, 303 (2004) 990-993.

[30] K.W. Desmond, E.R. Weeks, Influence of particle size distribution on random close packing of spheres, Physical Review E, 90 (2014).

[31] J.D. Bernal, J. Mason, Co-Ordination of Randomly Packed Spheres, Nature, 188 (1960) 910-911. 
[32] G.H. Koenderink, H. Zhang, D.G. Aarts, M.P. Lettinga, A.P. Philipse, G. Nägele, On the validity of Stokes-Einstein-Debye relations for rotational diffusion in colloidal suspensions, Faraday discussions, 123 (2003) 335-354.

[33] G.L. Hunter, E.R. Weeks, The physics of the colloidal glass transition, Reports on Progress in Physics, 75 (2012).

[34] Y.M. Joshi, Dynamics of colloidal glasses and gels, Annual review of chemical and biomolecular engineering, 5 (2014) 181-202.

[35] M. Hermes, M. Dijkstra, Jamming of polydisperse hard spheres: The effect of kinetic arrest, EPL (Europhysics Letters), 89 (2010) 38005.

[36] So Much More to Know \&\#x85, Science, 309 (2005) 78-102.

[37] T.E. Angelini, E. Hannezo, X. Trepat, M. Marquez, J.J. Fredberg, D.A. Weitz, Glass-like dynamics of collective cell migration, Proceedings of the National Academy of Sciences, 108 (2011) 4714-4719.

[38] W.H. Wang, Dynamic relaxations and relaxation-property relationships in metallic glasses, Progress in Materials Science, 106 (2019) 100561.

[39] D. Turnbull, Under what conditions can a glass be formed?, Contemporary physics, 10 (1969) 473-488.

[40] P.G. Debenedetti, F.H. Stillinger, Supercooled liquids and the glass transition, Nature, 410 (2001) 259-267.

[41] P.N. Pusey, W. van Megen, Observation of a glass transition in suspensions of spherical colloidal particles, Phys Rev Lett, 59 (1987) 2083-2086.

[42] B. Zhang, X. Cheng, Structures and dynamics of glass-forming colloidal liquids under spherical confinement, Physical review letters, 116 (2016) 098302.

[43] P. Pusey, Colloidal glasses, Journal of Physics: Condensed Matter, 20 (2008) 494202.

[44] K.V. Edmond, M.T. Elsesser, G.L. Hunter, D.J. Pine, E.R. Weeks, Decoupling of rotational and translational diffusion in supercooled colloidal fluids, Proceedings of the National Academy of Sciences, 109 (2012) 1789117896. 
[45] E.R. Weeks, J.C. Crocker, A.C. Levitt, A. Schofield, D.A. Weitz, Threedimensional direct imaging of structural relaxation near the colloidal glass transition, Science, 287 (2000) 627-631.

[46] E.R. Weeks, P. Habdas, Confocal microscopy and the colloidal glass transition., Abstracts of Papers of the American Chemical Society, 226 (2003) U290-U290.

[47] L. Berthier, G. Biroli, Theoretical perspective on the glass transition and amorphous materials, Reviews of modern physics, 83 (2011) 587.

[48] L. Janssen, Mode-coupling theory of the glass transition: A primer, Frontiers in Physics, 6 (2018) 97.

[49] E.R. Weeks, D.A. Weitz, Properties of cage rearrangements observed near the colloidal glass transition, Phys Rev Lett, 89 (2002) 095704.

[50] M. Kim, S.M. Anthony, S.C. Bae, S. Granick, Colloidal rotation near the colloidal glass transition, The Journal of chemical physics, 135 (2011) 054905.

[51] C.R. Nugent, K.V. Edmond, H.N. Patel, E.R. Weeks, Colloidal glass transition observed in confinement, Physical Review Letters, 99 (2007).

[52] R.E. Courtland, E.R. Weeks, Direct visualization of ageing in colloidal glasses, Journal of Physics-Condensed Matter, 15 (2003) S359-S365.

[53] E.R. Weeks, D.A. Weitz, Subdiffusion and the cage effect studied near the colloidal glass transition, Chemical Physics, 284 (2002) 361-367.

[54] Z. Zheng, F. Wang, Y. Han, Glass transitions in quasi-two-dimensional suspensions of colloidal ellipsoids, Physical review letters, 107 (2011) 065702.

[55] J. Roller, A. Laganapan, J.-M. Meijer, M. Fuchs, A. Zumbusch, Observation of liquid glass in suspensions of ellipsoidal colloids, Proceedings of the National Academy of Sciences, 118 (2021).

[56] S. Vivek, E.R. Weeks, Decoupling of translational and rotational diffusion in quasi-2D colloidal fluids, The Journal of chemical physics, 147 (2017) 134502.

[57] V.N. Manoharan, M.T. Elsesser, D.J. Pine, Dense packing and symmetry in small clusters of microspheres, Science, 301 (2003) 483-487. 
[58] S.C. Glotzer, M.J. Solomon, Anisotropy of building blocks and their assembly into complex structures, Nature materials, 6 (2007) 557-562.

[59] L.C. Hsiao, S. Pradeep, Experimental synthesis and characterization of rough particles for colloidal and granular rheology, Current Opinion in Colloid \& Interface Science, 43 (2019) 94-112.

[60] S. Jamali, J.F. Brady, Alternative frictional model for discontinuous shear thickening of dense suspensions: Hydrodynamics, Physical review letters, 123 (2019) 138002.

[61] H. Shi, S. Roy, T. Weinhart, V. Magnanimo, S. Luding, Steady state rheology of homogeneous and inhomogeneous cohesive granular materials, Granular matter, 22 (2020) 14.

[62] M.D. Haw, Colloidal suspensions, Brownian motion, molecular reality: a short history, Journal of physics: condensed matter, 14 (2002) 7769.

[63] J.C. Crocker, E.R. Weeks, Particle tracking using IDL, Retreived from http://www. physics. emory. edu/faculty/weeks//idl, (2011).

[64] J.C. Crocker, D.G. Grier, Methods of digital video microscopy for colloidal studies, Journal of colloid and interface science, 179 (1996) 298-310. 



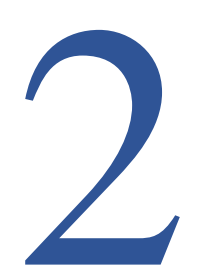

\section{A Method for Reversible Control over Nano-roughness of Colloidal Particles*}

Colloidal particles often display a surface topography that is smooth down to the nanometer scale. Introducing roughness at this length scale can drastically change the colloidal interactions, adsorption at interfaces and bulk flow behavior. We report on a novel, simple method to induce and control nano-scale roughness on (water-based) polymer latex colloids. Reducing the amount of dissolved gases in the aqueous phase from the electrolyte solution surrounding the particles generates self-structured surface asperities with an amplitude that can be tuned via temperature and repetition of the treatment. Due to the viscoelastic nature of the polymeric asperities, a mild thermal treatment below the glass transition temperature can be used for nanostructure relaxation so that the particles can recover their original topography, making this method fully reversible. Roughness can thus be controlled without affecting the chemical composition of the colloidal surface. Experiments for varying particle size, polymer type and surface chemistry suggest broad applicability of our method.

\footnotetext{
*The research described in this chapter has been published as Ilhan, B., Annink, C., Nguyen, D. V., Mugele, F., Sîretanu, I., \& Duits, M. H. (2019). A method for reversible control over nanoroughness of colloidal particles. Colloids and surfaces A: Physicochemical and engineering aspects, 560, 50-58.
} 


\subsection{Introduction}

Particles with controlled surface roughness are important for both fundamental science and industrial applications. Introducing (or enhancing) surface heterogeneity on colloids has significant outcomes for such systems, especially for the interactions between the particles. When two colloids come close together, the thermodynamic (e.g., electrostatic, van der Waals) forces will be strongly modified due to the presence of the asperities (or pits) [1-3]. In the presence of non-adsorbing polymers that fit in the nano-scale surface cavities of the rough particles, attractive depletion forces between the colloids will be strongly altered as well [4]. Particle interactions induced by (shear) flow can also be strongly modified by surface roughness. For example, short-ranged hydrodynamic forces will change because of alterations in the lubrication film thickness [5]. If the particles approach each other even closer to the point of direct contact, friction forces come into play. These forces generally depend strongly on surface topology, surface roughness. Even roughness with a relatively small amplitude can significantly change the diffusive and rheological behaviors, leading to strong suppression of rotational diffusion [6] and a (dramatic) shear thickening behavior [7-9] with increased particle concentration. Also, at interfaces, the behavior of rough particles is very different from that of smooth ones; roughness can cause enhanced adsorption barriers at liquid-liquid interfaces [10] and modified capillary interactions $[11,12]$ once the particles have been adsorbed. These modified interactions at the particle level correspondingly have consequences at a more collective level, e.g., the stability of Pickering emulsions [13].

Also, granular systems can be strongly influenced by particle surface roughness. Examples are avalanches or fluidized beds, where the constitutive particle dynamics is dependent on the surface topology [14]. In tribology, particle surface contact mechanics rely on the effective contact area, which depends on roughness and load force [15]. Consequently, the degree of roughness has implications for friction and wear [15]. Nano-scale surface roughness is also a key parameter for modifying the contact forces between particles $[14,16,17]$ and likewise, the roughness strongly affects the adhesion force between surfaces $[17,18]$.

While solid particles with geometrical surface heterogeneity have captured the attention of scientists already for a long time, the past decade has seen a strongly revived interest in this topic, in conjunction with the increased availability of rough colloidal particles. Many new systems and corresponding preparation 
methods have seen the light. One group of methods involves modifying the synthesis of polymer-based colloids. Adjusting the dosage of crosslinker in dispersion polymerizations was shown to effectively control the surface roughness of obtained particles [19, 20]. Another polymer-based method is to swell particles with liquid and then partially deswell them, to create liquid protrusions [21]. A different group of methods introduces roughness on inorganic solid particles. One of them involves partial removal of material from the outer regions of a solid particle via etching [9, 22]. Besides these approaches, there also exist other methods which are applicable to both polymer-based and solid-based colloids. One is the hetero-aggregation of different-sized colloids. This process is usually controlled via surface charge. To tailor the desired roughness amplitude, big core particles and small aggregating shell particles can be selected [23-27]. Another one is the single-step sol-gel method employing functionalized polymer particles as a template to nucleate solid nanoparticles on their surface [28, 29].

These methods, while being successful, also have their specific drawbacks. Hetero-aggregation methods require multiple steps and require good control over aggregation and stabilization. The 'chemical contrast' (material, surface charge) between the core particle and the surface bumps may be significant, which can be a secondary drawback in cases where the roughness needs to be controlled without changing the surface chemistry. Methods that use leaching or crosslinking of the particle's outer regions offer more simplicity and limited control over the type of roughness geometry. Along with that, they also pose constraints in preserving the initial sphericity of the particle. Finally, a limitation shared by all mentioned methods is that the process is irreversible. The ability to remove the surface roughness again while preserving the material can be interesting for both fundamental studies (studying the same sample at different roughness amplitudes) and applications (e.g., in switching between solid and liquid-like behavior of a colloidal suspension via the surface roughness).

This chapter describes a novel and simple method to induce roughness on particles in a reversible way and without introducing chemical heterogeneity. Our method builds on a well-documented procedure to roughen macroscopic polymer surfaces (e.g., films of polystyrene (PS), polymethyl methacrylate (PMMA), polytetrafluoro ethylene (PTFE)) [30-32]. Use is made of the viscoelastic nature of the polymers well below the glass transition temperature. Key roles in such nano-structuration have been attributed to local electric fields generated by the present ions and the enhanced mobility at the surface region of the (otherwise glassy) polymer [30-32]. It has also been pointed out that the electric field on the 
polymer surface depends critically on the density of charges and notably on the separation between the charges and the interface. Displacing the position of the ions by as little as a few Angstrom can entail a dramatic change in the electric field on the polymer and thereby enable or disable the nano-structuration. Also, changes in the density of ions in a very thin layer adjacent to the polymer could contribute to changes in the local electric field. Degassing of the aqueous solution is supposed to achieve more intimate contact between water and polymer surface and thus facilitate the nano-structuration [31-34]. This means that a simple degassing procedure can induce roughness in polymer films.

Transfer of this method to polymer-based colloids like PS or PMMA, PTFE is not entirely trivial since the colloidal particles must always contain an additional chemical functionality to stabilize them against coagulation; typically charged groups originating from the molecules used for initiating the polymerization. This chapter explores the induction of surface asperities on diverse types of polymer latex particles (mostly commercial ones) with varying particle sizes and surface groups. Systematic variation of the treatment conditions is applied to capture the tunable roughness amplitude. In addition, the possibility of reversing the nanostructuration via thermal treatment is explored.

\subsection{Materials and Methods}

\subsubsection{Materials}

Polystyrene (PS) and Poly Methyl Methacrylate (PMMA) colloids with different sizes and surface charges were used in our experiments; see Table 2.1. All purchased colloids were used as received. Systems PS-500 and PS-1k were cleaned by three cycles of centrifugation/redispersion in water. An atactic PS polymer sample of $250 \mathrm{~kg} / \mathrm{mol}\left(\mathrm{T}_{\mathrm{g}}=103^{\circ} \mathrm{C}\right)$ was obtained from ACROS Organics and toluene from Sigma Aldrich. Oxidized Silicon $\left(\mathrm{Si} / \mathrm{SiO}_{2}\right)$ wafers were obtained from Okmetic. 
Table 2.1. Specifications of the particles used in this study.

\begin{tabular}{|c|c|c|c|c|}
\hline Sample & $\begin{array}{c}\text { Diameter } \\
{[\mathrm{nm}]}\end{array}$ & Polymer & Functionality & Manufacturer \\
\hline PS-100 & 100 & PS & $\begin{array}{l}\text { sulfate, orange } \\
\text { fluorescent }\end{array}$ & Sigma Aldrich \\
\hline PS-227 & 227 & PS & $\begin{array}{c}\text { sulfate, yellow/green } \\
\text { fluor. }\end{array}$ & Molecular Probes \\
\hline PS-500 & 500 & PS & sulfonate modified & $\begin{array}{c}\text { www.sprakellab.nl } \\
{[35]}\end{array}$ \\
\hline PS-1K & 1000 & PS & $\begin{array}{l}\text { hydroxylated } \\
\text { ('plain') }\end{array}$ & Micromod \\
\hline PM-1K & 1000 & PMMA & $\begin{array}{c}\text { hydroxylated } \\
\text { ('plain') }\end{array}$ & Micromod \\
\hline PS-10K & 10000 & PS & carboxyl modified & Molecular Probes \\
\hline
\end{tabular}

Aqueous solutions of the reactant $\mathrm{HNO}_{3}$ were prepared from $0.1 \mathrm{~N}$ stock solution (Sigma Aldrich). Post reaction $\mathrm{pH}$-neutralization of some suspensions was done with $0.1 \mathrm{~N} \mathrm{NaOH}$ solution (Sigma Aldrich). MilliQ water (Millipore) with a conductivity of $18 \mathrm{M} \Omega . \mathrm{cm}$ was used for all the steps involving the preparation of solutions and the (re)dispersion of the particles.

\subsubsection{Method of Inducing Asperities}

Rough colloids were prepared by adapting a recent method [31-34] for creating roughness on thin polymer films. In this method, a film of polymer, spin-coated on a silicon wafer or mica, is exposed to a degassed aqueous solution of a specific salt, acid or base at a selected temperature below $\mathrm{T}_{\mathrm{g}}$. This exposure creates longlasting nanostructuration that remains when the degassed aqueous solution is replaced by pure water, or the sample is removed from the solution. The characteristic size and density of the self-assembled nanostructure (roughness of polymer surface) can be tuned via the ion concentration and type [31], the concentration of dissolved gases [32] and the temperature during the exposure step [33]. In the case of colloids, additional aspects come into play: the polymer material is now dispersed, which requires colloidal stability to be maintained throughout the procedure. In the starting material, this stability is provided by functional surface groups (not present on the films) but degassing, and exposure to dissolved electrolytes can compromise it. The degassing of particle suspensions has been observed to produce foam bubbles that remain for a long 
time, while the exposure of colloids to high electrolyte concentrations is known for its potential to cause coagulation. To avoid these issues, we exposed a small volume of pristine colloids to a relatively large amount of freshly degassed aqueous solution at the selected acid concentration and temperature; the composition and temperature of the mixture are then largely set by the exposing solution. Additionally, the duration of the exposure to a concentrated electrolyte environment was kept low.
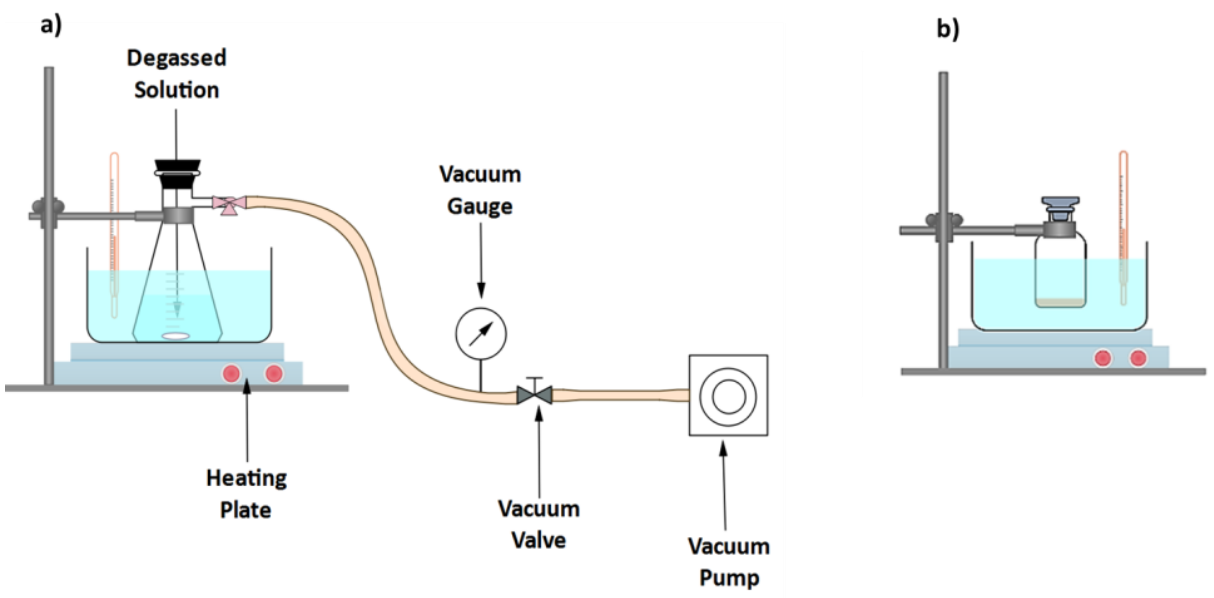

Figure 2.1. Schematic diagram of the experimental system. a) degassing procedure, b)thermal equilibration of the colloidal suspension before exposing it to the degassed solution.

Our procedure is illustrated in Figure 2.1 and explained in more detail below. The first step comprises the preparation of a degassed electrolyte solution in MilliQ water. In the present study, we show results for $\mathrm{HNO}_{3}$ as the electrolyte. The concentration hereof was defined via the $\mathrm{pH}$, which was adjusted to $1.25+0.05$ (corresponding to $\sim 55 \mathrm{mM}$ ) for all samples using the $0.1 \mathrm{~N} \mathrm{HNO}_{3}$ stock. $200 \mathrm{ml}$ of reactant solution was prepared in a Büchner Erlenmeyer loaded with a magnetic stirring bar. The Erlenmeyer was sealed with a rubber stop cock and connected to a vacuum line via an adjustable 2-way valve. A dual-stage rotary pump (Edwards RV5) was used to create the vacuum. The pressure inside the Erlenmeyer was monitored using an electronic vacuum gauge (BrandTech DCP3000) and regulated via the vacuum valve. The temperature was controlled by fully immersing the Erlenmeyer in a water bath placed on a hotplate. Degassing was carried out under vigorous magnetic stirring to promote the 
nucleation of air bubbles. The formation of vapor bubbles (i.e. boiling) was avoided by adjusting the pressure to stay above the boiling point at the given setpoint temperature. $\mathrm{HNO}_{3}$ solutions were first degassed at room temperature $\left(19-22^{\circ} \mathrm{C}\right)$ for 2 hours at $\sim 12 \mathrm{mbar}$, where typically bubble formation was visible in the first 30 minutes. Subsequently, the solutions were brought at setpoint conditions $\left(25^{\circ} \mathrm{C} / 15 \mathrm{mbar}\right.$ or $\left.45^{\circ} \mathrm{C} / 55 \mathrm{mbar}\right)$ and kept there for 30 minutes under a continuous vacuum. In parallel, the pristine colloids were prepared for the reaction by pipetting $200 \mu \mathrm{l}$ of suspension at $5 \%$ wt. into a sealed $100 \mathrm{ml}$ glass bottle. This bottle was placed inside the used water bath and kept there for 5 minutes for thermal equilibration. The small amount of suspension was observed to remain liquid-like during this time. Then, $100 \mathrm{ml}$ of the degassed solution was transferred to the bottle containing the colloids. This transfer was done quickly $(\approx 5-10 \mathrm{sec}$.) after removing the vacuum via the rubber stop cock, to minimize the re-dissolution of air. After sealing the bottle and hand-shaking the mixture, the polymer colloids were in contact with the degassed water solution of $\mathrm{pH} 1.25$ for 10 min, during which the suspension was sonicated (at the same temperature) to ensure good dispersion. To preserve the colloidal stability of all the treated suspensions, the duration of the exposure to high electrolyte concentration was minimized. For that reason directly after taking the sample from ultrasonic bath, 3 cycles of centrifugation in a Mistral $3000 \mathrm{E}$ at $3200 \mathrm{rpm}$ (corresponding to $\sim 2400 \mathrm{~g}$ ) and redispersion with MilliQ water were carried out. In the case of relatively smaller-sized particles $(<<500 \mathrm{~nm}$ diameter) where the centrifugation times needed to be much longer, a loss of colloidal stability was observed after the first centrifugation at low $\mathrm{pH}$. Thereby, this issue was resolved in subsequent preparations by neutralizing the low $\mathrm{pH}$ environment with $0.1 \mathrm{~N} \mathrm{NaOH}$ directly after the nanostructuration step. The last centrifuged sediment was redispersed in $100 \mathrm{ml}$ water, giving a final suspension concentration of $0.01 \mathrm{wt} \%$. Visual inspections for sedimentation and measurements of the hydrodynamic diameter with dynamic light scattering (Malvern Zetasizer Nano ZS) confirmed that stable suspensions were obtained under all reported conditions.

We also performed experiments with dry substrates. Two types were used: i) deposited colloidal particles and ii) spin-coated pure polymer films, both on oxidized $\mathrm{Si} / \mathrm{SiO}_{2}$ wafers. The use of deposited rather than suspended particles is a more practical way of exploring different preparation conditions. In further discussions, adsorbed and suspended particles will be distinguished via labels ($\mathrm{A}$ and $-\mathrm{S})$. We verified that both types of preparations produce comparably similar roughness in terms of RMS amplitude: the example in Figure A1 (in Appendix) illustrates this. The spin-coated pure polymer films were used as a 
reference sample; this was useful for diagnostic purposes and is recommended for future applications of our method. In successful preparations, both the film and the particles showed a (roughly comparable) roughness. Cases in which neither the film nor the particles (treated in parallel) showed any roughness were taken as an indication for improper degassing. Cases where only the film had become roughened were not encountered but would have indicated a specific matter with the colloid. For instance, functional surface charge groups on particles to stabilize them against coagulation can interfere with the process of nanostructuration by altering the adsorption of ions at the interface. Also, any usage of crosslinking agents during the synthesis of polymer colloids will affect the mobility of the surface layer, which is detrimental to nanostructuration.

For these dry substrates, the preparation of the degassed electrolyte solution was the same. i) Deposition of particles was done by dispensing a $\sim 22 \mu 1$ droplet of a 0.5 wt \% suspension onto the $\mathrm{Si} / \mathrm{SiO}_{2}$ wafer and letting it dry in air. ii) Polymer films were obtained by dissolving $250 \mathrm{kDa}$ PS in toluene up to $8.75 \mathrm{wt} \%$ and spin-coating at $1000 \mathrm{rpm}$ for 5 seconds and right after $5000 \mathrm{rpm}$ for 1 minute. This procedure was followed by annealing the coated wafers in an oven at $95^{\circ} \mathrm{C}$ for 12 hours. For both samples i) and ii) the coated $\mathrm{Si} / \mathrm{SiO}_{2}$ wafers were thermally equilibrated by placing them in an oven at the reaction temperature $\left(25^{\circ}-45^{\circ} \mathrm{C}\right)$ for 30 minutes. Then after degassing the $\mathrm{HNO}_{3}(\mathrm{pH}=1.25)$ solution, these wafers were carefully immersed in $100 \mathrm{ml}$ of the degassed solution and kept inside for $\approx 10$ minutes. After this, wafers were collected from the flask and gently dried with nitrogen flow.

In some experiments, the colloids were exposed to the degassed solution more than once (to enhance the size of the asperities and their corresponding surface coverage). In these cases, a fresh amount of $\mathrm{HNO}_{3}$ solution was degassed before re-exposing the (-A type) samples. In case the repeated reaction was carried out at an elevated temperature, the colloids were not preheated to the setpoint temperature anymore to avoid the thermal relaxation of the already formed surface bumps. Otherwise, all procedural steps were the same as for the pristine colloids.

\subsubsection{Characterization of Surface Topography}

Quantitative characterization of both smooth and rough particle surfaces was conducted with tapping mode atomic force microscopy using either a Multimode AFM (Bruker) or a Dimension Icon AFM (Bruker). All samples were analyzed 
in air using cantilevers from Mikro Masch, NSC36, with a spring constant of 0.6 $\mathrm{N} / \mathrm{m}$ and a sharp Si tip $\left(\mathrm{R}_{\text {tip }}<10 \mathrm{~nm}\right)$. For the analysis of dispersed particles, $20 \mu \mathrm{l}$ of the $0.01 \mathrm{wt} \%$ suspension was pipetted on top of a $\mathrm{Si} / \mathrm{SiO}_{2}$ wafer and left to dry under ambient conditions before AFM imaging.

\subsubsection{Calculation of Roughness Parameter}

Surface roughness was quantified in different ways. The first one was calculating the root-mean-square (RMS) amplitude from the AFM (height) images. The RMS parameter represents the standard deviation of the height profile belonging to the surface asperities. $\mathrm{z}(\mathrm{x}, \mathrm{y})$ profiles, exported from the AFM recordings, were analyzed using a custom-developed MATLAB program. The apical zones (subtending typically $10 \%$ of the total area) of individual particles were fitted with smooth spherical profiles, using least squares regression to obtain the average radius and center location for each particle. Subsequently, the radial component of the spherical fit was subtracted from the distance between the center of the smooth profile and each $(\mathrm{x}, \mathrm{y}, \mathrm{z})$ surface location. The resulting roughness amplitudes (in the radial direction) $\Delta r(x, y)$ were then used to calculate the rms per particle:

$$
\mathrm{RMS}=\sqrt{\frac{\sum_{\mathrm{i}}^{\mathrm{N}} \mathrm{z}_{\mathrm{i}}^{2}}{\mathrm{~N}}}
$$

where $\mathrm{N}$ denotes the number of points, typically ranging between $6 \times 10^{4}$ and $2.6 \times 10^{5}$ per particle. This method is illustrated in Figure 2.2.a and 2.b.

Additionally, maximum peak-to-valley heights $\left(\mathrm{R}_{\max }\right)$ were calculated from $\Delta \mathrm{r}(\mathrm{x})$ values. $R_{\max }$ corresponds to the maximum height difference. Its definition is given by eq. (2.2) where $\Delta r_{\text {peak }}$ and $\Delta r_{\text {valley }}$ denote the maximum peak height and valley depth accordingly. This parameter provides information complementary to the RMS value; e.g., the Rmax would be much larger than the RMS value in case of a low asperity density.

$$
\mathrm{R}_{\max }=\Delta \mathrm{r}_{\text {peak }}+\left|\Delta \mathrm{r}_{\text {valley }}\right|
$$

Considering that the roughness landscape is 3 dimensional (see Figures. 2.2c and $2 \mathrm{~d}$ ), there needs to be another quantification to characterize lateral roughness 
aspects (e.g. the width of the surface asperities or the density of the surface asperities on the particles). One general way to capture this aspect is to calculate the 'developed interfacial area ratio' denoted as $\mathrm{S}_{\mathrm{dr}}$ [36]. This parameter indicates the percentage of the additional surface area contributed by the texture compared to an ideal smooth plane (covering the same $(\mathrm{x}, \mathrm{y})$ measurement region). Its definition is given in eq. (2.3), where $\mathrm{A}_{\text {rough }}$ corresponds to the area of the surface with asperities $\Delta r(x, y)$ and $A_{\text {projected }}$ corresponds to the projected area of the same surface for the smooth case.

$$
\mathrm{S}_{\mathrm{dr}}=\frac{\mathrm{A}_{\text {rough }}-\mathrm{A}_{\text {projected }}}{\mathrm{A}_{\text {projected }}}
$$

$\mathrm{S}_{\mathrm{dr}}$ is affected by the texture amplitude and by the width and spacing of the asperities. Considering that the presented method results in self-induced asperities exhibiting diversities in height profiles and lateral length scales and surface coverage density (Figure.2.2), this measure is complementary to the RMS and $R_{\max }$ parameters in that it is more sensitive to the lateral length scale of the asperities. $S_{\mathrm{dr}}$ values were extracted from Nanoscope software. All samples were characterized at three different spots (i.e., particle apexes). 


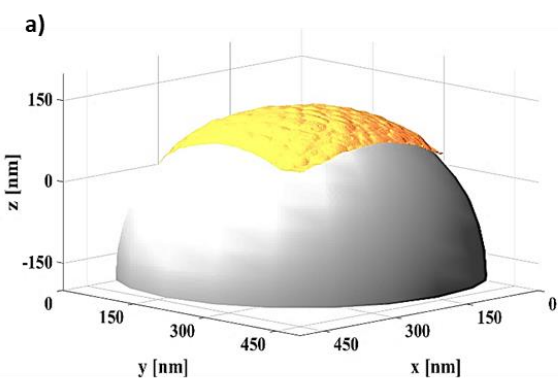

b)

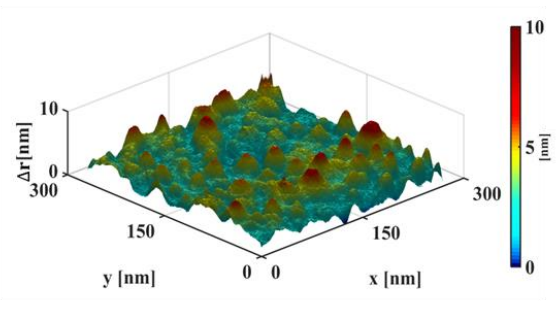

c)

d)
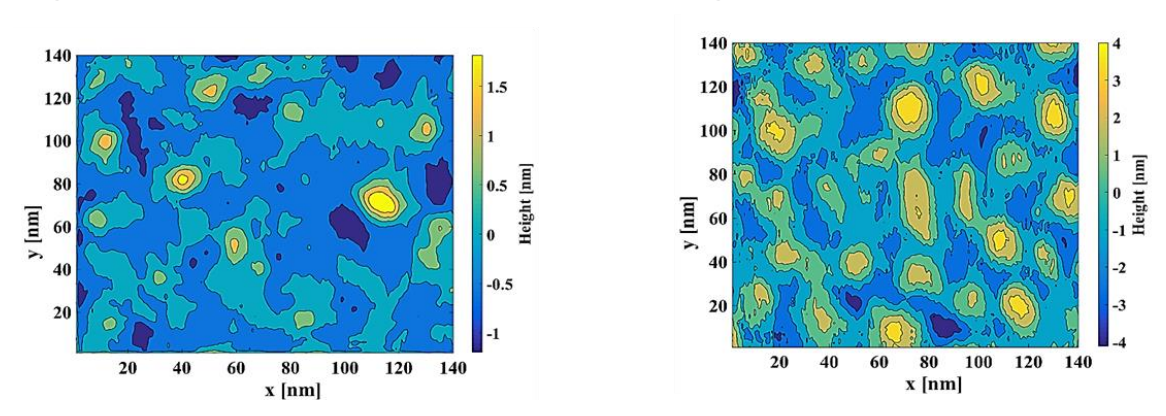

Figure 2.2. a) AFM scan and corresponding Matlab surface of a roughened $500 \mathrm{~nm}$ PS particle and the fit that best captures the apex of the spherical profile, b) residual height profile $\Delta \mathrm{r}(\mathrm{x}, \mathrm{y})$ after spherical background subtraction. c,d) contour plots (after background subtraction) for PS-227 particles roughened to different extents with different conditions: (c) at $25^{\circ} \mathrm{C}$ and (d) at $45^{\circ} \mathrm{C}$ after the second treatment.

\subsection{Results}

The creation of surface asperities on polymer latex particles by exposure to degassed aqueous electrolyte solution turned out to work well for a variety of systems with good reproducibility. Figure 2.3 shows a typical result for $500 \mathrm{~nm}$ size PS particles with and without exposure to degassed $\mathrm{HNO}_{3}(\mathrm{pH}=1.25)$ solution. Both the SEM and the AFM images show particles that are smooth before and rough after the treatment. Moreover, the SEM images also demonstrate a good level of uniformity of the surface roughness within each particle set. However, SEM is not suitable for accurately quantifying the roughness due to a lack of 3D localization and the limited microscope resolution. AFM has superior capabilities in this respect and was therefore used as the standard for surface roughness characterizations. 

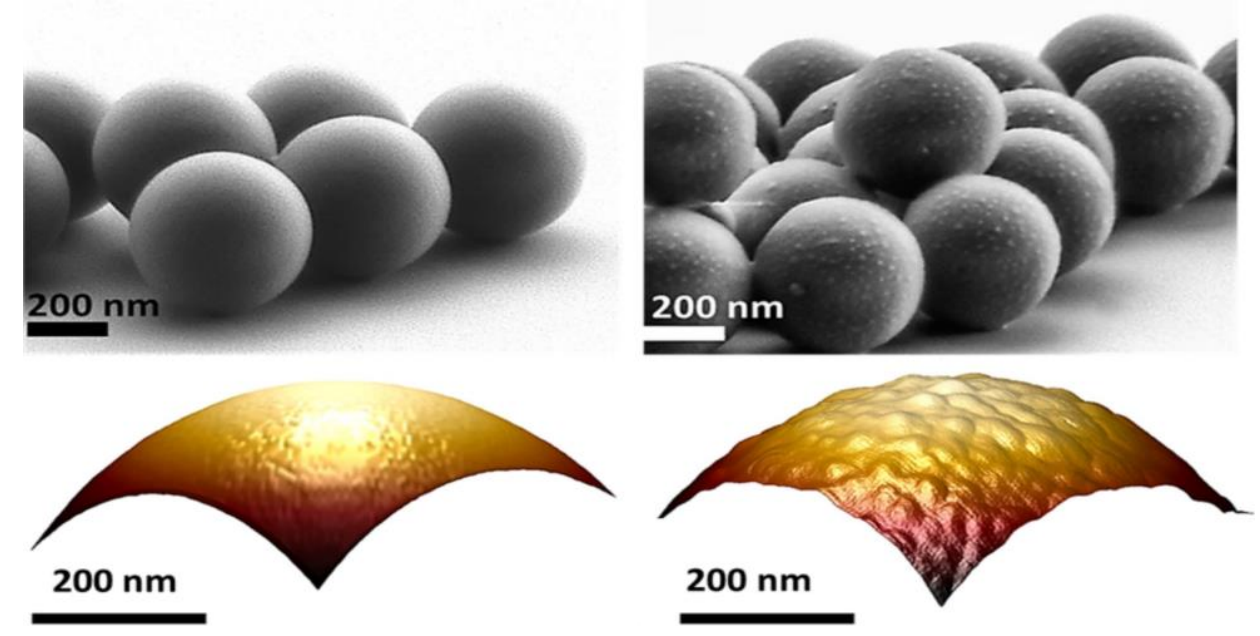

Figure 2.3. Images of colloid PS-500-A before and after being treated with degassed $\mathrm{HNO}_{3}$ solution at $45^{\circ} \mathrm{C}$ Top row: SEM images. Bottom row: AFM images (note that scale bar for AFM apex images is for $\mathrm{x}-\mathrm{y}$ axis, $\mathrm{z}$ axis is rescaled 0.8 factor.

$\mathrm{HNO}_{3}$ (at $55 \mathrm{mM}, \mathrm{pH}$ 1.25) was used as the electrolyte in the current work. Variables were the temperature of the reaction mixture and the number of repetitions of the treatment. A typical outcome is illustrated for the $227 \mathrm{~nm}$-sized PS particles in Figure 2.4. The formation of surface asperities is directly visible for colloids treated with degassed $\mathrm{HNO}_{3}$ solution at $25^{\circ} \mathrm{C}$ (compare Figure 2.4.a and $\mathrm{b}$ ). If the reaction temperature is set higher at $45^{\circ} \mathrm{C}$, the roughness increases: the asperities become larger in height and diameter, leading to an increase in their surface coverage (Figure 2.4.c). The most dramatic surface topography change is obtained after giving the colloids two subsequent treatments. Here we demonstrate the effect of repetition at $45^{\circ} \mathrm{C}$ (Figure 2.4.d). Both the surface coverage and the size of the individual asperities in terms of peak-to-valley distances are largest here. However, a third exposure to the degassed solution did not further increase the roughness amplitude. This may be due to the limited spatial extent over which a free surface polymer layer is more mobile than the bulk polymer. All the trends mentioned above are confirmed by the calculated roughness parameters in Table 2.2. Each of these indicators increases on going from case Figure 2.4.a to case Figure 2.4.d. Similar trends for particle systems with different sizes can be seen in Figure. A3 a-b (Appendix). 
a)

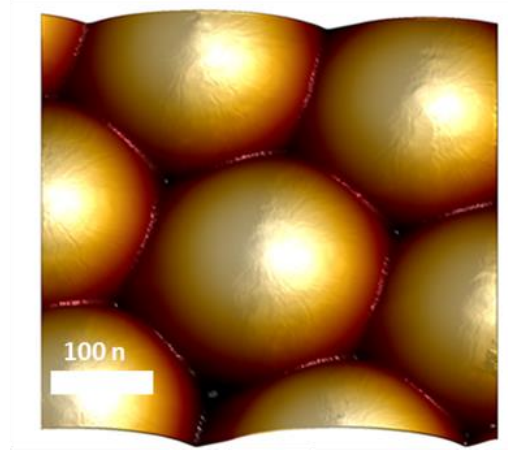

c)

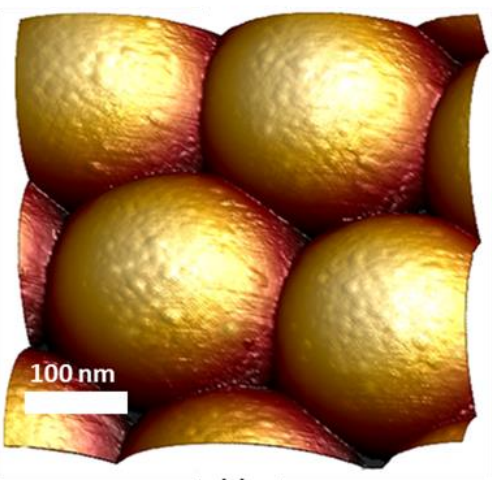

b)

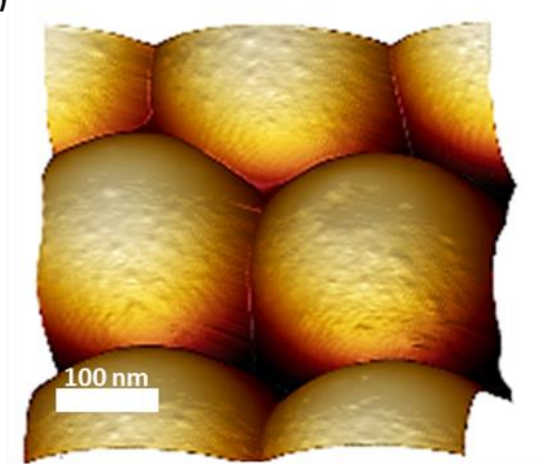

d)

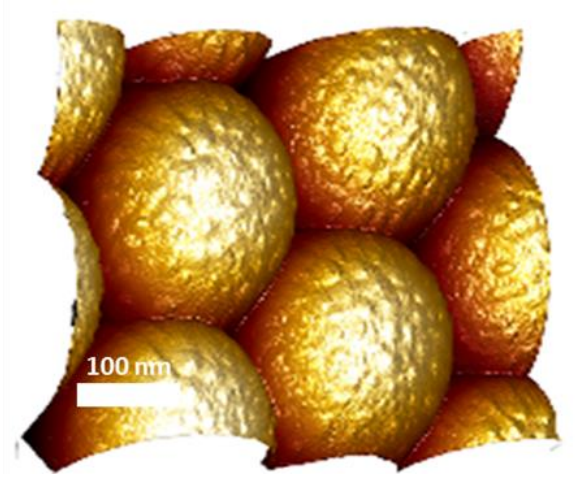

Figure 2.4. Surface topography of the PS-227-A system after different treatments: a) none, b) degassed at $25^{\circ} \mathrm{C}, \mathrm{c}$ ) degassed at $45^{\circ} \mathrm{C}$, d) degassed twice at $45^{\circ} \mathrm{C}$.

Table 2.2. RMS, Rz and Sdr values for the PS- 227 system after different treatments. Data correspond to Figure 2.4.

\begin{tabular}{c|ccc} 
Condition & $\mathrm{RMS}[\mathrm{nm}]$ & $\mathrm{R}_{\max }[\mathrm{nm}]$ & $\mathrm{S}_{\mathrm{dr}}[\%]$ \\
\hline as received & 0.28 & - & 0.036 \\
$25^{\circ} \mathrm{C}$ & $0.41-\mathrm{S}$ & $2.82-\mathrm{S}$ & $0.59-\mathrm{S}$ \\
$1^{\text {st }}$ time $45^{\circ} \mathrm{C}$ & $1.05-\mathrm{S}$ & $4.75-\mathrm{S}$ & $3.20-\mathrm{S}$ \\
$2^{\text {nd }}$ time $45^{\circ} \mathrm{C}$ & $2.87-\mathrm{A}$ & $6.27-\mathrm{A}$ & $15.5-\mathrm{A}$
\end{tabular}

We also assess the reproducibility of our nanostructuration method. The data presented in Table 2.2 comprise averages over three apexes of the particles for the represented condition (for standard deviations, see Figure A2, in Appendix). In addition, the experiments are repeated at the same conditions, and the spread 
among three experiments is typically $\sim 10 \%$ (at most $13 \%$ for $227 \mathrm{~nm}$ ) of the RMS parameter; this is much smaller than the differences between the treatment conditions. An overview of repeated experiments can be found in Table A2.

In Figure 2.5, we visually compare PS latex systems having different sizes and surface groups. The reaction conditions were the same: all four systems were twice exposed to degassed $\mathrm{HNO}_{3}(\mathrm{pH}=1.25)$ solution at $45^{\circ} \mathrm{C}$ (i.e., the procedure that gives the largest RMS values). Clearly, significant surface roughness is obtained for each of the systems. A quantitative roughness analysis from AFM images of particles shown in Figure 2.5 and others (not shown here) are presented in Table 2.3 and Table 2.4 .

a)

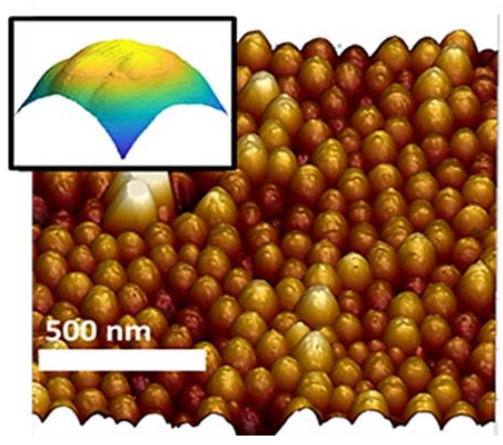

c)

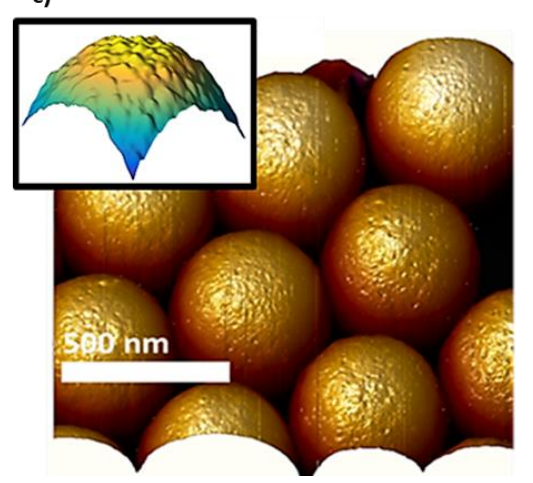

b)

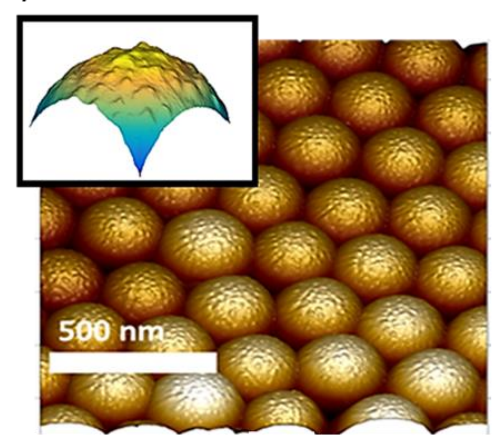

d)

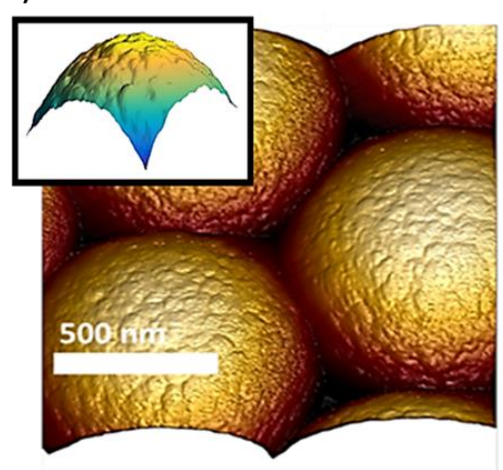

Figure 2.5. AFM surface characterization of different systems, all treated twice at $45^{\circ} \mathrm{C}$. Insets show a typical particle taken from the main image (scaling ratio of axes: 1:1:0.5 for $x: y: z)$. a) PS-100-A, b) PS-227-A, c) PS-500-A, d) PS-1k-A. 
Table 2.3. Surface roughness characterization for various PolyStyrene systems exposed to different reaction conditions. $-\mathrm{A}$ and $-\mathrm{S}$ indicate whether particles were adsorbed or suspended. 'As is' refers to the original suspension, while PS-film refers to a spincoated film. RMS and $\mathrm{R}_{\max }$ data are given in $\mathrm{nm}$.

\begin{tabular}{l|ccccccc} 
& RMS & RMS & RMS & RMS & $\mathrm{R}_{\max }$ & $\mathrm{R}_{\max }$ & $\mathrm{R}_{\max }$ \\
& as is & $25^{\circ} \mathrm{C}$ & $45^{\circ} \mathrm{C}$ & $2 \times 45^{\circ} \mathrm{C}$ & $25^{\circ} \mathrm{C}$ & $45^{\circ} \mathrm{C}$ & $2 \times 45^{\circ} \mathrm{C}$ \\
\hline PS-100 & 0.14 & $0.37-\mathrm{A}$ & $0.41-\mathrm{A}$ & $0.92-\mathrm{A}$ & $1.49-\mathrm{A}$ & $2.09-\mathrm{A}$ & $3.67-\mathrm{A}$ \\
PS-227 & 0.28 & $0.41-\mathrm{S}$ & $1.05-\mathrm{S}$ & $2.87-\mathrm{A}$ & $2.82-\mathrm{S}$ & $4.75-\mathrm{S}$ & $6.27-\mathrm{S}$ \\
PS-500 & 0.35 & $0.53-\mathrm{S}$ & $0.71-\mathrm{S}$ & $2.16-\mathrm{A}$ & $3.23-\mathrm{S}$ & $3.36 \mathrm{~S}$ & $9.51-\mathrm{A}$ \\
PS-1k & 0.44 & $1.02-\mathrm{S}$ & $1.51-\mathrm{S}$ & $3.57-\mathrm{A}$ & $6.08-\mathrm{S}$ & $7.45-\mathrm{S}$ & $12.6-\mathrm{A}$ \\
PS-10k & 2.81 & n.a. & $7.94-\mathrm{A}$ & $17.2-\mathrm{A}$ & n.a. & $35.6-\mathrm{A}$ & $76.3-\mathrm{A}$ \\
PS film & 0.22 & 1.14 & 1.65 & 2.17 & 5.26 & 12.2 & 17.1
\end{tabular}

Table 2.4. Surface roughness characterization in terms of $S_{\mathrm{dr}}$ (given as a \%). $-\mathrm{A}$ and $-\mathrm{S}$ indicate whether particles were adsorbed or suspended

\begin{tabular}{l|llll} 
& \multicolumn{1}{|l}{$\mathrm{S}_{\mathrm{dr}}$} & $\mathrm{S}$ & $\mathrm{S}$ & $\mathrm{S}$ \\
& & & & \\
& as is & $25^{\circ} \mathrm{C}$ & $45^{\circ} \mathrm{C}$ & $2 \times 45^{\circ} \mathrm{C}$ \\
\hline PS-100 & 0.027 & $0.24-\mathrm{A}$ & $0.88-\mathrm{A}$ & $2.71-\mathrm{A}$ \\
PS-227 & 0.036 & $0.59-\mathrm{S}$ & $3.20-\mathrm{A}$ & $15.5-\mathrm{A}$ \\
PS-500 & 0.041 & $0.46-\mathrm{S}$ & $1.02-\mathrm{S}$ & $8.25-\mathrm{A}$ \\
PS-1k & 0.034 & $0.61-\mathrm{S}$ & $2.88-\mathrm{S}$ & $4.36-\mathrm{A}$ \\
PS-10k & 0.100 & n.a. & $1.18-\mathrm{A}$ & $18.1-\mathrm{A}$ \\
PS film & 0.22 & 1.14 & 1.65 & 2.17
\end{tabular}

From these tables, several trends can be observed. Looking at the effect of treatment conditions (as done before for PS-227) reveals that for all systems, $\mathrm{RMS}, \mathrm{R}_{\max }$ and and $\mathrm{S}_{\mathrm{dr}}$ increases changing from $25^{\circ} \mathrm{C}$ to $45^{\circ} \mathrm{C}$ and/to $45^{\circ} \mathrm{C}-(2 \mathrm{x})$. The comparison between particles with different sizes and surface functional groups at the same treatment condition is slightly less clear. If we assume that the size is the most important difference between the particles, an increase in the obtained roughness with particle size could be identified in most cases (an exception being the PS-500 nm system). Generally, the trends with particle size are more evident for the RMS and $R_{\max }$ values than for the $S_{d r}$ value. The 
roughness parameters for the PS film generally lie closest to those of the PS-1k system.

a)

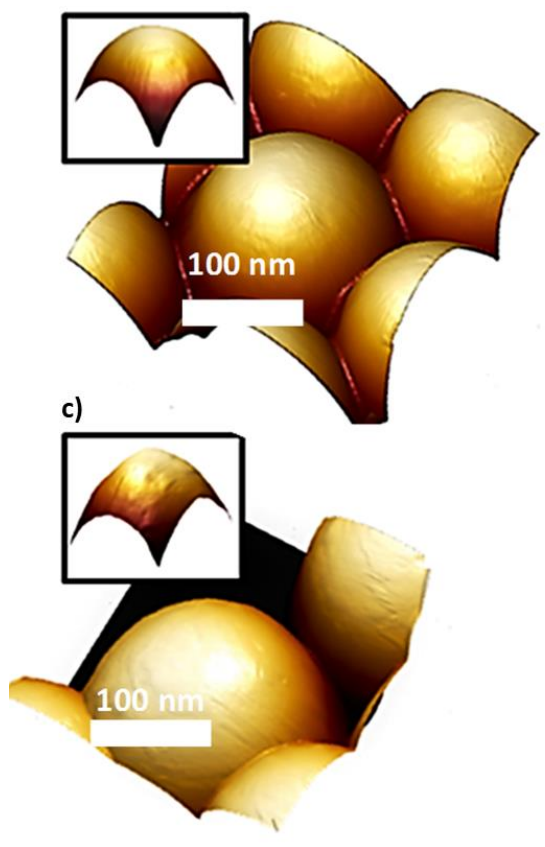

b)

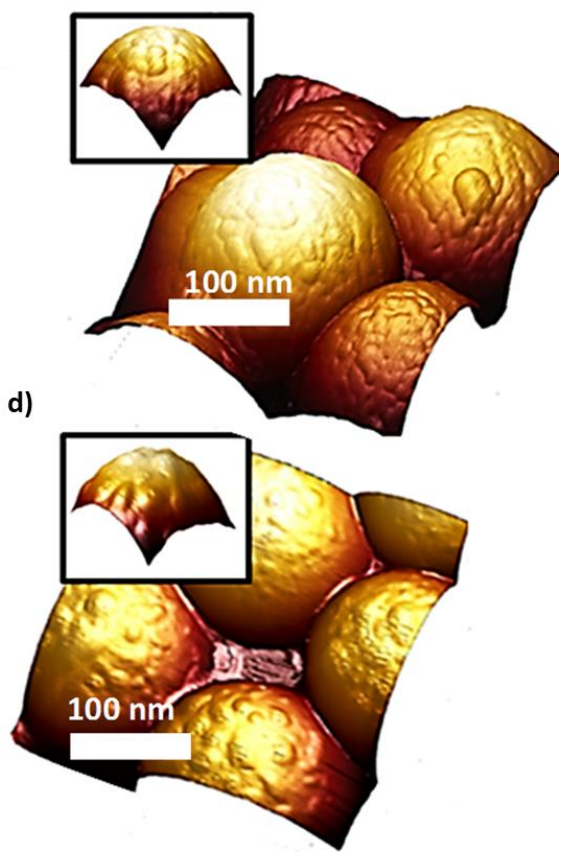

Figure 2.6. Analysis of the thermo-reversibility of the nanostructuration explored for system PS-227-S. The transition from a) to b) was achieved by exposing the particles to the degassed solution at $45^{\circ} \mathrm{C}$. c) shows the same sample after exposure to $75^{\circ} \mathrm{C}$ for $12 \mathrm{~h}$. d) shows the result of subsequent treatment at $45^{\circ} \mathrm{C}$.

Finally, we explore the possible reversibility of the asperities formation. Given the polymeric nature of the particle surface, spontaneous reversal might be possible: polymer chains in the vicinity of a 'free surface' are known to have enhanced mobility [37-39], possibly leading to relaxation at timescales that are relevant to the experimentalist. Two scenarios were considered: storage at low temperature $\left(4^{\circ} \mathrm{C}\right)$, to achieve a long shelf life and annealing at an elevated temperature but still below the bulk glass temperature $\left(75^{\circ} \mathrm{C}\right)$. No degassing was applied during these relaxation experiments. AFM analysis of PS-227-S stored in the refrigerator at $+4^{\circ} \mathrm{C}$ showed no significant change in surface topography for 11 weeks (see Figure. A4). The results of the first roughening and subsequent annealing of the same particle system at $75^{\circ} \mathrm{C}$ can be found in Figure 2.6.a-6.c. 
After treating the particles with degassed $\mathrm{HNO}_{3}$ solution at $45^{\circ} \mathrm{C}$, they obtain a visible surface roughness, as before $(a, b)$. It should be emphasized here that thermal treatment does not create surface roughness in the absence of degassing. Subsequently annealing the particles at $75^{\circ} \mathrm{C}$ for 12 hours makes the roughness features disappear (see Figure 2.6c). Finally, as shown in Figure. 2.6.d, the surface nanostructure reappears if the colloids are exposed again to degassed $\mathrm{HNO}_{3}$ solutions at $45^{\circ} \mathrm{C}$ (For details, see Figure A4 and A5 in the Appendix).

\subsection{Discussion}

Our results show that the surface roughness of several (PS) colloids can be induced in a controlled way by exposing them to degassed $(55 \mathrm{mM}, \mathrm{pH}=1.25)$ $\mathrm{HNO}_{3}$ solution, at a chosen temperature between $25^{\circ}$ and $45^{\circ} \mathrm{C}$ and for a chosen number of times. The scope of the method appears to be much wider than the use of PS particles and $\mathrm{HNO}_{3}$ electrolyte; although most experiments were done under this condition, also salts [30-34] and PMMA particles (see Figure. A6 in Appendix) were observed to create surface roughness in comparable experiments. Considering the similarities with polymer films' roughening, other materials like PTFE colloids might also be suitable substrates.

Additionally, we have demonstrated that nanostructuration is both possible for colloidal particles and dry particles just as well (by performing the type -A experiments). In this respect, our method also proposes a simple methodology for altering the topography of the dry particulate media for any future applications.

It should not be disregarded that, although this method is successful for colloids as well as flat films, the mechanistic details about what happens on the colloidal surfaces still need further clarification. As suggested in recent literature related to flat polymer films [30-32], nano-structuration involves three aspects; a mobile surface layer on the surface of the polymers, the presence (and concentration) of ions and the distance between the ions and this mobile surface. Once the amount of dissolved gas is reduced due to the vacuum exposure, the distance between ions and the colloid surface becomes smaller, generating a higher electric field near the surface of the polymer. Due to the existence of the mobile surface layer with properties deviating from their bulk (glassy) counterparts, the surface of the colloids can be restructured, even at temperatures well below $T_{g}$, as shown by our results. The effect of increased treatment temperature might be attributed to enhanced mobility as well as the enhanced thickness at the outer region of the 
polymer surfaces, leading to stronger nanostructuration (roughness amplitude). Currently, the existence of the mobile surface layer on polymer colloids is intensively studied, but a possible quantitative description is still a daunting challenge [37-39]. Considering the ease of our method, it could be possible to employ this method in further studies to confirm and measure such mobile layers on polymer colloidal surfaces by making use of the time scales of asperity formation and relaxation.

It should also be noted that, unlike polymer films, colloids contain functional surface groups, which might influence the nano-structuration process. The density and chemical environment of these surface groups were not among the controls in the present study. Therefore, it cannot be excluded that PS particles with comparable size but prepared under different conditions will show somewhat different roughness topologies. The 'deviating' data for the PS-500 system in Table 2.2 might be attributed to local differences in chemical architecture (e.g., chain density, crosslink density, functional groups) near the particle surface. Our most important finding is that the trends with treatment conditions (temperature, repetition) are expected to remain the same for other systems.

Compared to other existing methodologies, our method is relatively simple. Another advantage of our method is that it is possible to tailor the roughness characteristics of polymer latex particles for a wide range of sizes, covering a size range from $10 \mu \mathrm{m}$ to (as small as) $100 \mathrm{~nm}$. The relative (i.e., normalized by particle radius) amplitude of the rms roughness lies in the range of $0.3-2.5 \%$. This is comparable to other systems in which the particles are roughened without growing added material onto them. The maximum roughness amplitude obtained with $\mathrm{HNO}_{3}$ as the agent appears to be correlated with the particle size (see Table 3). This might indicate that the thickness of the mobile outer layer on particles increases with increasing particle size. It might still be possible to increase the roughness more via further optimization of the preparation conditions, such as treatment temperature, ion concentration and type. However, we think that ultimately vertical amplitude of the roughness will be limited by the extent of the free surface polymer layer on the particles.

A distinctive advantage of our simple, water-based method is the possibility to reverse the creation of rough surface topography. By making use of the viscoelastic nature of the polymer colloids and treatments with temperatures below $\mathrm{T}_{\mathrm{g}}$, surface roughness can be created or evened on demand (leaving the 
surface chemistry largely intact) by setting the right temperature (and air pressure). This possibility to change the surface topography in situ could make our method useful in studies where a comparison between smooth and rough particles is needed without changing the sample (concentration) (e.g., studies into shear-thickening), and in applications where on-demand changes in surface topography are used to create functionality (e.g., systems that rely on particle jamming).

The surface roughness was compared using RMS amplitudes, $\mathrm{R}_{\max }$ and developed interfacial area ratio $\left(\mathrm{S}_{\mathrm{dr}}\right)$. The thermodynamic and hydrodynamic interactions between rough colloids might depend on more than the height and surface density of the asperities. Our quantification of other surface structural parameters might be useful for future correlations with behaviors like, e.g., diffusion, interfacial shear phenomena, contact area mechanics and flow behavior of colloidal systems [36].

\subsection{Conclusion}

We presented a methodology for creating nano-sized, reversible surface roughness on aqueous polymer latex colloids. The approach was adapted from a similar methodology developed for flat polymer films. Implementation of this method on colloids was successful for a variety of particle sizes and surface groups. Variation of temperature and repetition of the treatment allowed (semi) quantitative control over roughness. Compared to other methods, our procedure offers a relatively simple way to obtain surface heterogeneity without introducing chemical inhomogeneity. Moreover, with our method, the surface roughness can be created and annihilated on demand by making use of thermo-reversibility.

\section{Acknowledgments}

This work was financially supported by NWO-CW (ECHO grant 712.016.004). We thank Martien Cohen Stuart for discussions, Dr. Joris Sprakel (Wageningen University) for providing us with the PS-500 system, Aram Klaassen and Alessandro Beltram for complementary AFM imaging, and Mark Smithers for SEM imaging. 


\section{Bibliography}

[1] J.Y. Walz, The effect of surface heterogeneities on colloidal forces, Adv Colloid Interfac, 74 (1998) 119-168.

[2] S. Bhattacharjee, C.-H. Ko, M. Elimelech, DLVO interaction between rough surfaces, Langmuir, 14 (1998) 3365-3375.

[3] J. Tsao, Role of surface roughness for colloidal interactions in aqueous media, Georgia Institute of Technology, 2016.

[4] M. Kamp, M. Hermes, C.M. van Kats, D.J. Kraft, W.K. Kegel, M. Dijkstra, A. van Blaaderen, Selective Depletion Interactions in Mixtures of Rough and Smooth Silica Spheres, Langmuir, 32 (2016) 1233-1240.

[5] H.J. Wilson, R.H. Davis, Shear stress of a monolayer of rough spheres, J Fluid Mech, 452 (2002) 425-441.

[6] L.C. Hsiao, I. Saha-Dalal, R.G. Larson, M.J. Solomon, Translational and rotational dynamics in dense suspensions of smooth and rough colloids, Soft Matter, 13 (2017) 9229-9236.

[7] L.C. Hsiao, S. Jamali, E. Glynos, P.F. Green, R.G. Larson, M.J. Solomon, Rheological State Diagrams for Rough Colloids in Shear Flow, Physical Review Letters, 119 (2017).

[8] C.P. Hsu, S.N. Ramakrishna, M. Zanini, N.D. Spencer, L. Isa, Roughnessdependent tribology effects on discontinuous shear thickening, Proceedings of the National Academy of Sciences of the United States of America, 115 (2018) 5117-5122.

[9] D. Lootens, H. van Damme, Y. Hemar, P. Hebraud, Dilatant flow of concentrated suspensions of rough particles, Phys Rev Lett, 95 (2005).

[10] A. San-Miguel, S.H. Behrens, Influence of Nanoscale Particle Roughness on the Stability of Pickering Emulsions, Langmuir, 28 (2012) 12038-12043.

[11] A. Ata, Y.I. Rabinovich, R.K. Singh, Role of surface roughness in capillary adhesion, J Adhes Sci Technol, 16 (2002) 337-346. 
[12] D. Stamou, C. Duschl, D. Johannsmann, Long-range attraction between colloidal spheres at the air-water interface: The consequence of an irregular meniscus, Phys Rev E, 62 (2000) 5263-5272.

[13] M. Zanini, I. Lesov, E. Marini, C.P. Hsu, C. Marschelke, A. Synytska, S.E. Anachkov, L. Isa, Detachment of Rough Colloids from Liquid-Liquid Interfaces, Langmuir, 34 (2018) 4861-4873.

[14] R. Wilson, D. Dini, B. Van Wachem, The influence of surface roughness and adhesion on particle rolling, Powder Technol, 312 (2017) 321-333.

[15] B. Bhushan, Contact mechanics of rough surfaces in tribology: multiple asperity contact, Tribol Lett, 4 (1998) 1-35.

[16] Y.I. Rabinovich, J.J. Adler, A. Ata, R.K. Singh, B.M. Moudgil, Adhesion between nanoscale rough surfaces - I. Role of asperity geometry, J Colloid Interf Sci, 232 (2000) 10-16.

[17] R. Fuchs, T. Weinhart, J. Meyer, H. Zhuang, T. Staedler, X. Jiang, S. Luding, Rolling, sliding and torsion of micron-sized silica particles: experimental, numerical and theoretical analysis, Granular Matter, 16 (2014) 281-297.

[18] C.Q. LaMarche, S. Leadley, P.Y. Liu, K.M. Kellogg, C.M. Hrenya, Method of quantifying surface roughness for accurate adhesive force predictions, Chem Eng Sci, 158 (2017) 140-153.

[19] D. Kim, D.Y. Lee, K. Lee, S. Choe, Effect of Crosslinking Agents on the Morphology of Polymer Particles Produced by One-Step Seeded Polymerization, Macromol Res, 17 (2009) 250-258.

[20] B. Peng, A. Imhof, Surface morphology control of cross-linked polymer particles via dispersion polymerization, Soft Matter, 11 (2015) 3589-3598.

[21] D.J. Kraft, W.S. Vlug, C.M. van Kats, A. van Blaaderen, A. Imhof, W.K. Kegel, Self-Assembly of Colloids with Liquid Protrusions, J Am Chem Soc, 131 (2009) 1182-1186.

[22] X. Du, J.H. He, A Self-Templated Etching Route to Surface-Rough Silica Nanoparticles for Superhydrophobic Coatings, Acs Appl Mater Inter, 3 (2011) 1269-1276. 
[23] M. Zanini, C.P. Hsu, T. Magrini, E. Marini, L. Isa, Fabrication of rough colloids by heteroaggregation, Colloid Surface A, 532 (2017) 116-124.

[24] C.L. Wang, J.T. Yan, X.J. Cui, H.Y. Wang, Synthesis of raspberry-like monodisperse magnetic hollow hybrid nanospheres by coating polystyrene template with Fe3O4@SiO2 particles, J Colloid Interf Sci, 354 (2011) 94-99.

[25] M. Chen, S.X. Zhou, B. You, L.M. Wu, A novel preparation method of raspberry-like PMMA/SiO2 hybrid microspheres, Macromolecules, 38 (2005) 6411-6417.

[26] X.M. Liu, J.H. He, Hierarchically structured superhydrophilic coatings fabricated by self-assembling raspberry-like silica nanospheres, J Colloid Interf Sci, 314 (2007) 341-345.

[27] E. Spruijt, H.E. Bakker, T.E. Kodger, J. Sprakel, M.A.C. Stuart, J. van der Gucht, Reversible assembly of oppositely charged hairy colloids in water, Soft Matter, 7 (2011) 8281-8290.

[28] Z. Qian, Z.C. Zhang, L.Y. Song, H.R. Liu, A novel approach to raspberrylike particles for superhydrophobic materials, J Mater Chem, 19 (2009) 12971304.

[29] X. Du, X.M. Liu, H.M. Chen, J.H. He, Facile Fabrication of Raspberry-like Composite Nanoparticles and Their Application as Building Blocks for Constructing Superhydrophilic Coatings, J Phys Chem C, 113 (2009) 9063-9070.

[30] I. Siretanu, Nanostructuration contrôlée de films de polymères, Bordeaux 1, 2011.

[31] I. Siretanu, J.P. Chapel, D. Bastos-Gonzalez, C. Drummond, Ions-induced nanostructuration: effect of specific ionic adsorption on hydrophobic polymer surfaces, J Phys Chem B, 117 (2013) 6814-6822.

[32] I. Siretanu, J.P. Chapel, C. Drummond, Water-ions induced nanostructuration of hydrophobic polymer surfaces, ACS Nano, 5 (2011) 29392947.

[33] I. Siretanu, H. Saadaoui, J.P. Chapel, C. Drummond, Spatial Heterogeneity of Glassy Polymer Films, Macromolecules, 48 (2015) 2787-2792. 
[34] I. Siretanu, J.P. Chapel, C. Drummond, Substrate Remote Control of Polymer Film Surface Mobility, Macromolecules, 45 (2012) 1001-1005.

[35] J. Appel, S. Akerboom, R.G. Fokkink, J. Sprakel, Facile One-Step Synthesis of Monodisperse Micron-Sized Latex Particles with Highly Carboxylated Surfaces, Macromolecular rapid communications, 34 (2013) 1284-1288.

[36] R. Leach, Characterisation of areal surface texture, Springer2013.

[37] Z. Fakhraai, J.A. Forrest, Measuring the surface dynamics of glassy polymers, Science, 319 (2008) 600-604.

[38] H. Kim, Y. Cang, E. Kang, B. Graczykowski, M. Secchi, M. Montagna, R.D. Priestley, E.M. Furst, G. Fytas, Direct observation of polymer surface mobility via nanoparticle vibrations, Nature Communications, 9 (2018).

[39] T. Sasaki, A. Shimizu, T.H. Mourey, C.T. Thurau, M.D. Ediger, Glass transition of small polystyrene spheres in aqueous suspensions, J Chem Phys, 119 (2003) 8730-8735. 


\section{Appendix}

Comparison (based on AFM imaging and analysis) between PS-227 colloids roughened by exposure to degassed $\mathrm{HNO}_{3}$ solution at $25^{\circ} \mathrm{C}$ with (-A) and (S) methods

a)

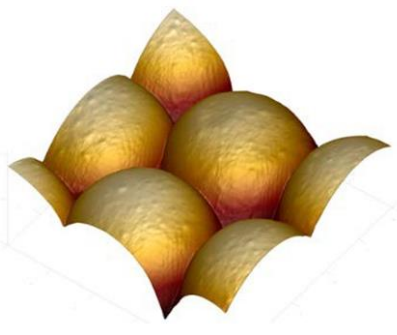

b)

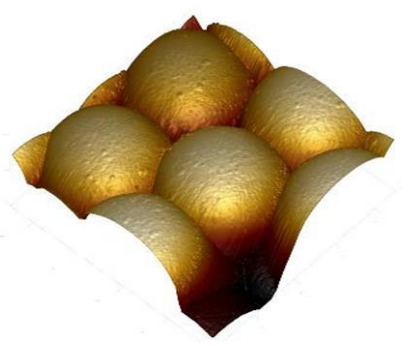

c)

\begin{tabular}{l|ll} 
PS-227 & $-\mathrm{A}$ & $-\mathrm{S}$ \\
\hline RMS (nm) & 0.44 & 0.41 \\
$\mathrm{~S}_{\mathrm{d}:}(\%)$ & 0.70 & 0.58
\end{tabular}

Figure A1. a) Particles adsorbed on a Si wafer (-A) and b) Particles in suspension (-S). Panel c) shows the roughness parameters.

Graphical representation of RMS roughness increase (including their standard deviation between apexes) with respect to the different treatment conditions.
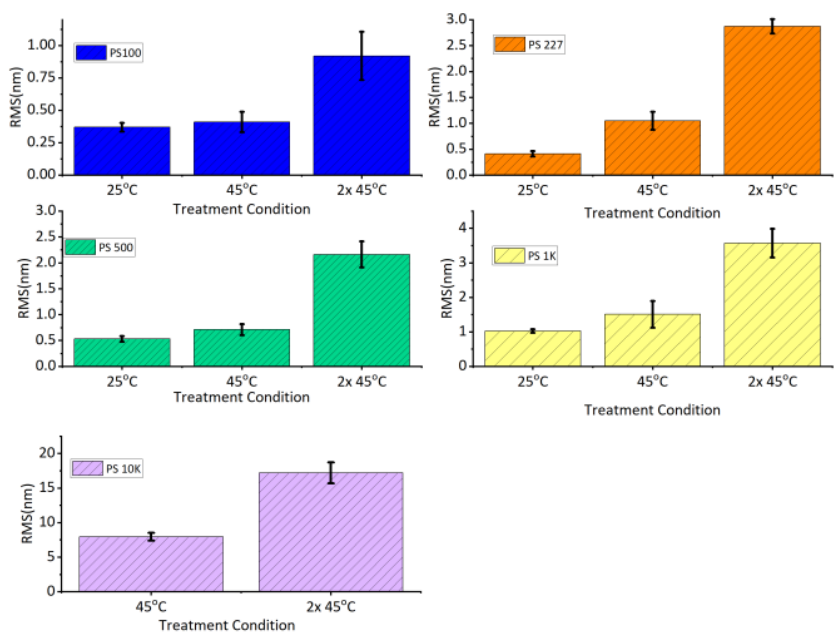

Figure A2. Corresponding Standard deviations for RMS values in Table 2.3. 
Demonstration of different treatment conditions $\left(25^{\circ} \mathrm{C}, 45^{\circ} \mathrm{C}, 2 \times 45^{\circ} \mathrm{C}\right)$ applied to particle systems with different sizes.

-PS film treatment is presented for a reference case, as described in Chapter 2.

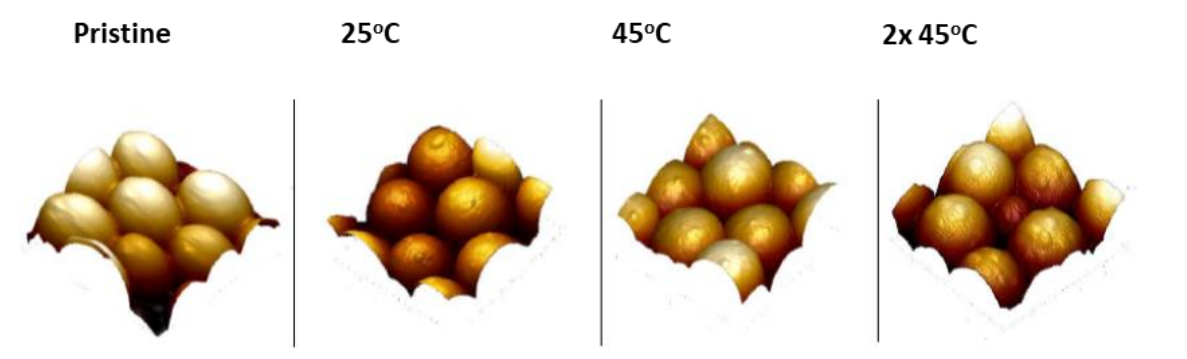

a) PS 100 colloids
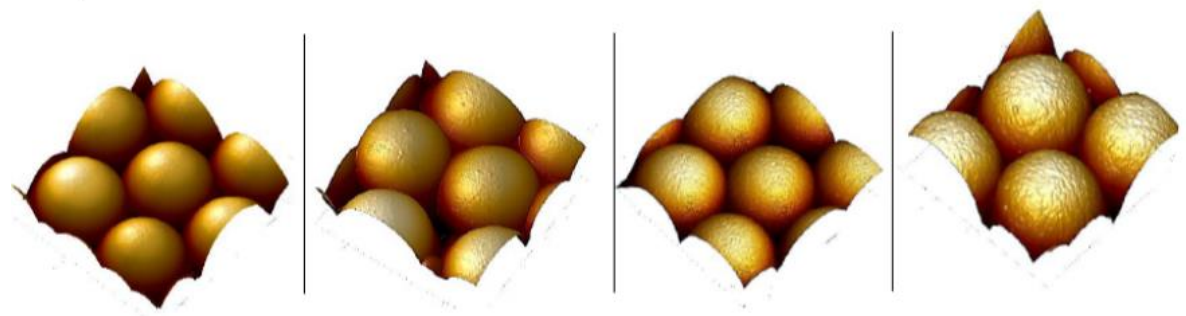

b) PS 500 colloids

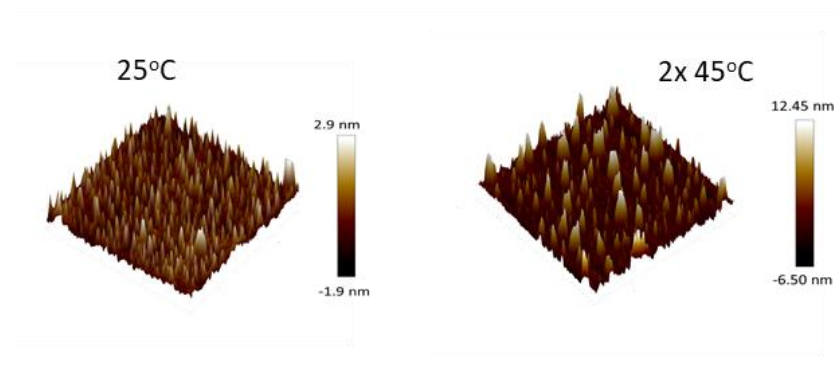

c) PS film treated at $25^{\circ} \mathrm{C}$ and $2 \times 45^{\circ} \mathrm{C}$

Figure A3. Variation of the surface topography of PolyStyrene with treatment conditions. Panels a) and b): PS-100-A respectively PS-500-A exposed to: i) no treatment, ii) degassing at $25^{\circ} \mathrm{C}$, iii) degassing at $45^{\circ} \mathrm{C}$, iv) degassing twice at $45^{\circ} \mathrm{C}$. Panel c): PS film $(\sim 1 \mathrm{x} 1 \mu \mathrm{m}$ area shown) exposed to 1$)$ degassing at $\left.25^{\circ} \mathrm{C}, 2\right)$ degassing twice at $45^{\circ} \mathrm{C}$. 


\section{Reproducibility of the Measurements}

Table A1 here provides an extensive data representation for all the measurements for different particle systems with different sizes. Conducted experiments at different conditions were repeated several times to ensure reproducibility of the results.

Table A1: Reproducibility of several treatments for PS-227-A and PS-500-A particles in terms of RMS, $R_{\max }$ and $S_{d r}$ values.

\begin{tabular}{l|lll}
\begin{tabular}{l|ll} 
PS-227-A \\
Exps.
\end{tabular} & $\mathbf{R M S}[\mathbf{n m}]$ & $\mathbf{R}_{\max }[\mathbf{n m}]$ & $\begin{array}{l}\mathbf{S}_{\mathbf{d r}} \\
{[\%]}\end{array}$ \\
\hline $25^{\circ} \mathrm{C}$ & 0.43 & 2.78 & 0.60 \\
$25^{\circ} \mathrm{C}$ & 0.57 & 3.54 & 0.78 \\
$25^{\circ} \mathrm{C}$ & 0.44 & 3.23 & 0.70 \\
$25^{\circ} \mathrm{C}$ & 0.41 & 2.82 & 0.59 \\
average & 0.46 & 3.09 & 0.67 \\
std. & 0.06 & 0.31 & 0.08 \\
\hline $45^{\circ} \mathrm{C}$ & 0.93 & 3.77 & 2.07 \\
$45^{\circ} \mathrm{C}$ & 0.97 & 4.56 & 4.57 \\
$45^{\circ} \mathrm{C}$ & 0.81 & 3.21 & 2.01 \\
$45^{\circ} \mathrm{C}$ & 1.05 & 4.75 & 3.2 \\
average & 0.94 & 4.07 & 2.96 \\
Std. & 0.09 & 0.62 & 1.04 \\
\hline $45^{\circ} \mathrm{C} 2 \mathrm{x}$ & 2.97 & 20.06 & 15.1 \\
$45^{\circ} \mathrm{C} 2 \mathrm{x}$ & 2.00 & 7.52 & 8.43 \\
$45^{\circ} \mathrm{C} 2 \mathrm{x}$ & 1.70 & 7.61 & 11.7 \\
$45^{\circ} \mathrm{C} 2 \mathrm{x}$ & 2.87 & 6.27 & 15.5 \\
average & 2.39 & 10.37 & 12.7 \\
Std. & 0.54 & 5.62 & 2.86 \\
\hline & & &
\end{tabular}


PS-500-A

\begin{tabular}{l|lll} 
Exps. & RMS [nm] & $\mathbf{R}_{\max }[\mathbf{n m}]$ & $\begin{array}{l}\mathbf{S}_{\mathbf{d r}} \\
{[\%]}\end{array}$ \\
\hline $25^{\circ} \mathrm{C}$ & 0.64 & 2.38 & 1.48 \\
$25^{\circ} \mathrm{C}$ & 0.70 & 3.23 & 1.03 \\
$25^{\circ} \mathrm{C}$ & 0.54 & 4.19 & 1.09 \\
$25^{\circ} \mathrm{C}$ & 0.41 & 4.25 & 0.42 \\
average & 0.60 & 4.07 & 1.01 \\
Std. & 0.11 & 0.77 & 0.389 \\
\hline $45^{\circ} \mathrm{C}$ & 0.82 & 2.51 & 2.16 \\
$45^{\circ} \mathrm{C}$ & 0.71 & 3.36 & 1.02 \\
average & 0.76 & 2.94 & 1.59 \\
Std. & 0.05 & 0.44 & 0.57 \\
\hline $45^{\circ} \mathrm{C} 2 \mathrm{X}$ & 1.81 & 7.01 & 8.65 \\
$45^{\circ} \mathrm{C} 2 \mathrm{x}$ & 2.24 & 7.98 & 9.52 \\
$45^{\circ} \mathrm{C} 2 \mathrm{x}$ & 2.57 & 9.43 & 7.06 \\
$45^{\circ} \mathrm{C} 2 \mathrm{x}$ & 2.16 & 9.51 & 11.4 \\
average & 2.20 & 8.48 & 9.16 \\
Std. & 0.27 & 1.05 & 1.57 \\
\hline & & & \\
\hline
\end{tabular}




\section{Temporal evolution and subsequent treatment results of the surface roughness of PS-227-S}

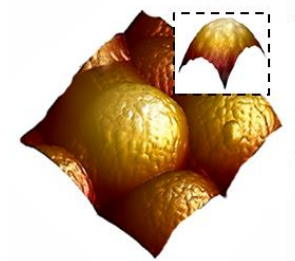

i) Initial RMS= $1.01 \mathrm{~nm}$

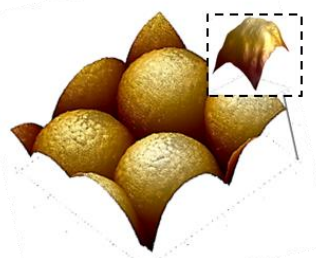

ii) 1 week: RMS $=0.83 \mathrm{~nm}$

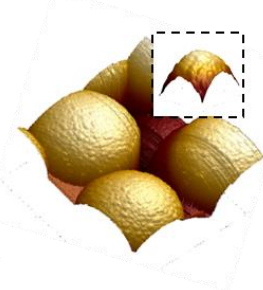

iii) 2 weeks: $\mathrm{RMS}=0.95 \mathrm{~nm}$

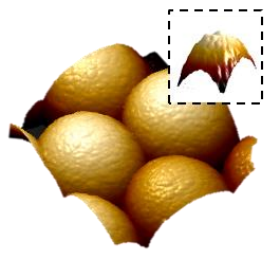

iv) 11 weeks: $\mathrm{RMS}=0.711 \mathrm{~nm}$

Figure A4. Left to right: after treating the system once at $45^{\circ} \mathrm{C}$, the fresh product was imaged with AFM. Next, the suspension was stored at $4^{\circ} \mathrm{C}$ and sampled after 1, 2 and 11 weeks for AFM imaging.

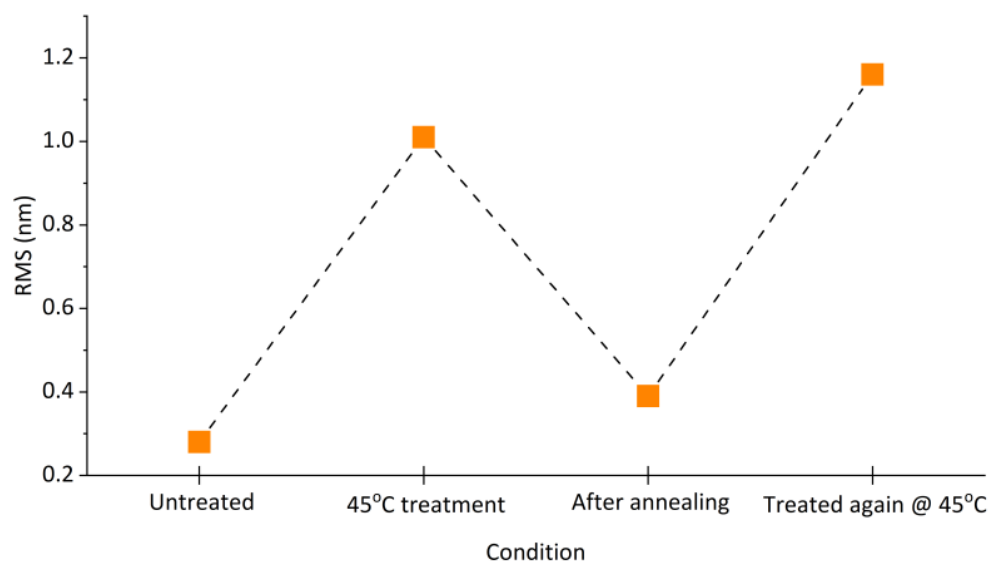

Figure A5. Surface roughness for PS-227 particles that were subsequently: i) treated at $45^{\circ} \mathrm{C}$, ii) annealed at $75^{\circ} \mathrm{C}$ and iii) treated again at $45^{\circ} \mathrm{C}$. 


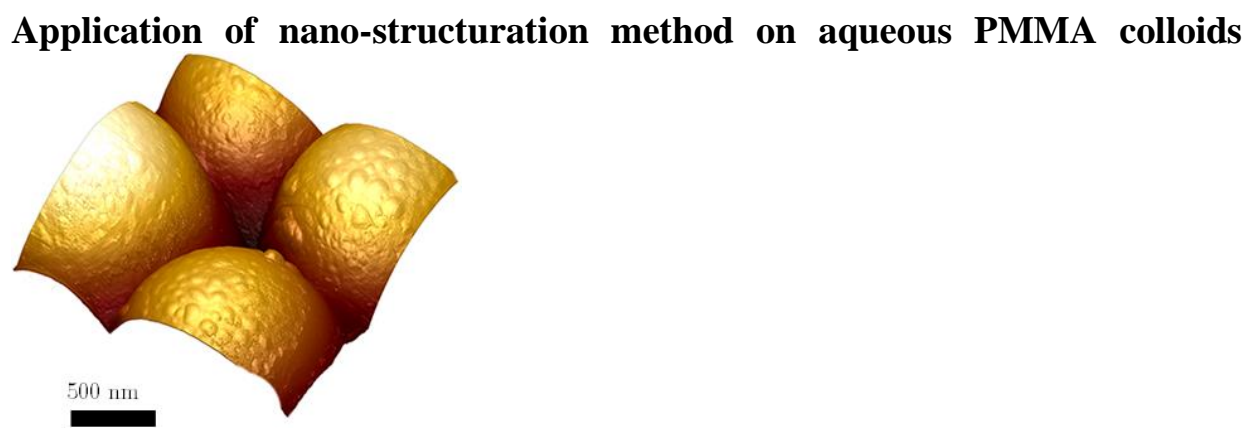

Figure A6. System PM-1k-A was exposed to degassed $\mathrm{HNO}_{3}$ solution at $45^{\circ} \mathrm{C}$, resulting RMS is $2.19 \mathrm{~nm}$. 



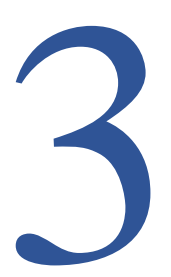

\section{Spherical Probes for Simultaneous Measurement of Rotational and Translational Diffusion in 3 Dimensions*}

Real-time visualization and tracking of colloidal particles with 3D resolution are essential for probing complex fluids' local structure and dynamics. Although tracking the translational motion of spherical particles is well-known, accessing the rotational dynamics of such particles remains a great challenge. Here, we report a novel approach of using fluorescently labeled raspberry-like colloids with an optical anisotropy to concurrently track translational and rotational dynamics in 3 dimensions. The raspberry-like particles are coated by a silica layer of adjustable thickness, which allows tuning the surface roughness. The synthesis and applicability of the proposed method are demonstrated by two types of probes: rough and smoothened. The accuracy of measuring Mean Squared (Angular) Displacements is also demonstrated by using these two probes dispersed in 2 different solvents. The presented 3D trackable colloids offer a high potential for a wide range of applications and studies, such as probing the dynamics of crystallization, phase transitions, biological interactions and the effect of surface roughness on diffusion.

\footnotetext{
*The research described in this chapter has been published as Ilhan, B., Schoppink, J. J., Mugele, F., \& Duits, M. H. (2020). Spherical probes for simultaneous measurement of rotational and translational diffusion in 3 dimensions. Journal of colloid and interface science, 576, 322-329.
} 


\subsection{Introduction}

Studying colloidal dynamics via time-resolved locations of individual particles can provide microscopic insight into various physical phenomena in different phases of matter [1-5]. Especially in systems with an intrinsic inhomogeneity, correlating the dynamics with the location inside the material can provide unique information that cannot be obtained with techniques that take an ensemble average over all particles or any other bulk method. Examples of research areas where local translational dynamics have been analyzed for this purpose include dense suspensions [6], glassy materials [7-9], polymer networks[10,11], spatially confined materials [12-15], food products [16], cell biology [17, 18] and virus infection mechanisms [19].

In contrast to the many studies on translational dynamics, research related to rotational dynamics is relatively scarce. This has been ascribed to a lack of experimental approaches for capturing rotational motion [20]. Rotational dynamics can shed a unique light onto various dynamic phenomena that cannot be accessed only with translational degrees of freedom, such as motion in glassy and supercooled states (where decoupling between translational and rotational diffusion emerges) [21-23]; particle adsorption and self-assembly at fluid interfaces [24, 25]; interfacial dynamics at solid-liquid interfaces [26] and biological interactions; such as viruses binding to membranes [27].

Ensemble averaged rotational diffusion of colloids has been studied by techniques such as fluorescence recovery after photobleaching (FRAP) [28, 29], depolarized dynamic light scattering [30] and nuclear magnetic resonance (NMR) spectroscopy [31]. These bulk methods fall short in identifying local (dynamic) heterogeneities. Measuring rotational diffusion via individual probe particles can provide valuable local information in terms of dynamic length scales and structural signatures of complex fluids, such as local defects and crosslink densities in polymer networks [32], local rheology of soft materials [33], or intrinsic features of active fluids in biochemical processes [34].

In recent years, different strategies have been used to track the rotational motion of diffusive probes of spherical and anisotropic particle shapes such as rods, ellipsoids, and particle clusters [35-39]. Here, geometrical anisotropy is widely utilized because it naturally provides an identifiable optical axis to track angular displacements [20]. The lack of such a natural frame of reference for spherical 
colloids requires a design where the optical isotropy is broken. A common type of such probes is the modulated optical nanoprobe (MOON) [40, 41]. This type of Janus particle usually consists of a fluorescent sphere that is half coated with a metal layer. Although these rotational probes attract interest in various fields [42], they also have some drawbacks. Due to the metal coating on one side, the surface chemistry is no longer uniform, and refractive index mismatches with the surrounding medium may compromise the image quality, especially for biological systems and high volume concentrations. Recently a new type of spherical rotational probe was introduced [23, 43]. These probes are bicolor or multicolor colloidal spheres with an eccentric core(s) shell structure, requiring multiple excitation wavelengths to be used. Although they provide homogenous surface chemistry, the core and shell centers may not coincide precisely. When connected cores with different labels are utilized, they have to be overgrown to a relatively large size to attain a (near) spherical overall shape.

In this chapter, we introduce a simple method for i) synthesizing fluorescently labeled raspberry-like spherical probes and ii) using them for simultaneously accessing rotational and translational dynamics in 3D. Our probes are made by densely covering a large silica $\left(\mathrm{SiO}_{2}\right)$ core with many small $\mathrm{SiO}_{2}$ particles, a fraction of which is fluorescently labeled. By coating these raspberries with a layer of silica of controllable thickness, we obtain particles of variable roughness while maintaining uniform surface chemistry. The optical anisotropy, introduced via the fluorescent tracers, allows for simultaneous tracking of each probe's translational and rotational motion in $3 \mathrm{D}$, using just one fluorescent dye. We demonstrate a proof of concept by tracking two types of probes (smoothened and rough) in the dilute regime and measuring the Mean Squared (Angular) Displacements.

\subsection{Materials and Methods}

\subsubsection{Materials}

TetraEthoxySilane, 25\% wt. Ammonia, analytical grade ethanol (99.9\%, Emsol), $1 \mathrm{~N}$ nitric acid solution, anhydrous dimethyl sulfoxide (>99.9\%) and glycerol (99.5\%) are purchased from Sigma Aldrich. All purchased chemicals are used as received. Deionized water (resistivity: $18 \mathrm{M} \Omega . \mathrm{cm}$ ) is obtained from a Millipore Synergy instrument. Core $\mathrm{SiO}_{2}$ particles $(\mathrm{r}=1.06 \mu \mathrm{m})$ with amine $\left(\mathrm{NH}_{2}\right)$ surface modification are purchased from Microparticles GmbH. Two types of smaller $\mathrm{SiO}_{2}$ particles (berries) are used: fluorescent particles (sicastar-greenF, plain, 
$\mathrm{r}=0.15 \mu \mathrm{m})$ are purchased from Micromod Partikeltechnologie $\mathrm{GmbH}$, while plain $\mathrm{SiO}_{2}$ particles are synthesized in house via the Stöber method [44, 45].

\subsubsection{Synthesis of Fluorescently Labelled Raspberries}

Raspberry particles are synthesized by coating the surface of positively functionalized (-NH2) $\mathrm{SiO}_{2}$ spheres (core, $\mathrm{r}=1.04 \pm 0.033 \mu \mathrm{m}$ ) with a dense layer of negatively charged small $\mathrm{SiO} 2$ spheres (berry, $\mathrm{r}=0.164 \pm 0.021 \mu \mathrm{m}$ ) via electrostatic heteroaggregation [46]. While the presence of (+) amine groups on the cores and (-) silanol ones on the berries already favor such aggregation, an optimization of the $\mathrm{pH}$ is needed to obtain interparticle bonds that are strong enough to prevent detachment by stirring forces. Meanwhile, also the stability (against homo-aggregation) has to be preserved for both cores and berries. Using $\mathrm{HNO}_{3}$ to adjust the $\mathrm{pH}$, Zeta potential measurements are conducted at varying $\mathrm{pH}$ for the aqueous dispersions of the particles. The results (shown in Appendix Figure A1) indicate an optimal $\mathrm{pH}$ of 4.5 , where $\zeta_{\text {core }}=+31 \mathrm{mV}$ and $\zeta_{\text {berry }}=-28 \mathrm{mV}$, $\zeta_{\text {fluo.berry= }}=21 \mathrm{mV}$.

Besides the zeta potentials, also the mixing ratio of the cores and berries has to be considered. The number of berry particles needed to ensure dense coverage on a core particle is estimated by calculating how many berry particles can be fitted into the shell space between a hypothetical sphere with a radius of [ $\left.R_{\text {core }}+2 R_{\text {berry }}\right]$ and a core particle. For our system, this calculation gives 386 berries per core. In practice, more berries are needed to ensure colloidal stability throughout the selfassembly process. Especially at the initial stages where the cores are only partially covered by berries, 'bridging aggregation' (berries binding to two cores) must be avoided. To prevent this, a ten times higher dose of berries is used.

To obtain optical anisotropy, a mixture of equally sized plain and fluorescent berries is used (see Figure 3.1.a), with a mixing ratio that typically leads to 4-5 fluorescent berries per particle. Assuming that all berries have an equal probability of being bonded to a core surface, the proportion of fluorescent berries is chosen to be $\sim 1.0 \%$. The hetero-aggregation is achieved by adding cores to a suspension of berry particles $(\sim 1 \% \mathrm{wt})$ under mild stirring and giving a reaction time of 1 hour. Excess berries are removed by three cycles of gravity settling and redispersion in an aqueous solution at $\mathrm{pH} 4.5$.

To preserve mechanical integrity and modify the surface roughness, a silica layer is overgrown onto these colloids via seeded growth [47]. Figure 3.1.b and 3.1.c 
show confocal fluorescence images of typical probes without/with fluorescent berries. Figures 3.1.d and 3.1.e show SEM and TEM images for two systems differing in the layer thickness and hence in final size (they are identical otherwise): Surface rough probes (RP) with an RMS roughness of $58 \mathrm{~nm}$ (4.5\% relative to average raspberry radius) and Smoothened probes (SP) with $23 \mathrm{~nm}$ RMS (1.5\% relative).
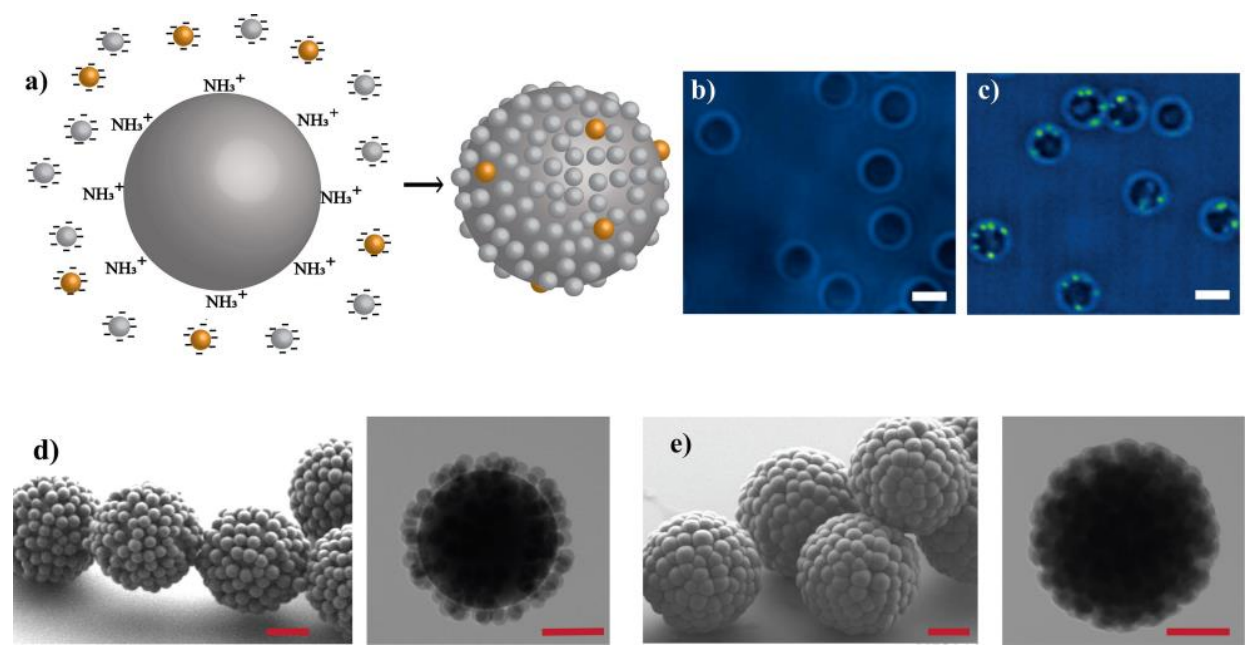

Figure 3.1. a) Illustration of the synthesis of the probe raspberries. b, c) superimposed brightfield and fluorescence images of raspberry probes without/with fluorescent berries (white scale bars are $2 \mu \mathrm{m}$ ), d, e) SEM and TEM images of rough (RP) and smoothened probes (SP) (red scale bars are $1 \mu \mathrm{m}$ ).

\subsubsection{Characterization Methods}

The hydrodynamic radii of the two berry systems, as measured with Dynamic Light Scattering (Malvern Zetasizer Nano ZS), are used to obtain equal sizes via seeded growth on the plain silica [48]. The size distributions of the cores, berries and probes are measured from TEM images (Philips CM300ST-FEG, $300 \mathrm{kV}$ ). Radii are measured from the fitted largest Feret diameter (ImageJ, FIJI). Core and berry size distributions are shown in Appendix Figure A2. SEM images are obtained with a Zeiss MERLIN HR-SEM. The mass density of the raspberry particles is measured with a pycnometer and found to be $1.62 \pm 0.06 \mathrm{~g} / \mathrm{cm}^{3}$.

The surface coverage of the berries on the raspberries is characterized with atomic force microscopy (AFM), (Dimension Icon, Bruker). Samples are analyzed in air 
using cantilevers from Mikro Masch, NSC36, with a spring constant of $0.6 \mathrm{~N} / \mathrm{m}$ and a sharp Si tip $\left(\mathrm{R}_{\mathrm{tip}}<10 \mathrm{~nm}\right)$. Surface roughness is quantified by the calculation of root-mean-square (RMS) amplitude from AFM height profiles. The apical zones of individual particles are fitted with a sphere via least squares regression by a custom-made Matlab program. Subsequently, the radial component of the spherical fit is subtracted from the distance between the center of the smooth profile and each $(\mathrm{x}, \mathrm{y}, \mathrm{z})$ surface location [49]. Details of this characterization can be found in Appendix Figure A6.

\subsubsection{Confocal Microscopy Experiments}

Raspberry particles are dispersed in refractive index matching solvents $\left(S_{1}\right.$ or $\left.S_{2}\right)$, starting from the water-based stock suspensions and using the known mass densities to calculate the mixing ratios [50]. The obtained dilute suspensions ( $\sim 0.3 \%$ by volume) are dispersed in custom-made cylindrical glass cuvettes of 6 $\mathrm{mm}$ diameter and bottom thickness of $\sim 170 \mu \mathrm{m}$. All measurements are conducted at $20^{\circ} \mathrm{C}$.

A VisiTech 'VT-infinity' Confocal Laser Scanning Microscope (CSLM) is used to capture image-time series. The CSLM unit is connected to a Nikon Eclipse TiU inverted microscope, a Hamamatsu digital camera (ORCA-flash 4.1, 100 fps) and a $100 \mathrm{~mW} 488 \mathrm{~nm}$ excitation laser source. A 100x oil immersion objective with a 1.49 numerical aperture is used to capture the images. Z-ranges spanning 6-12 $\mu \mathrm{m}$ are examined, where the lowest focal plane is set at $10 \mu \mathrm{m}$ above the glass bottom to avoid wall effects on the particle dynamics. Individual Z-stacks (i.e., time points in 3D localization) are captured using $1024 \mathrm{x} 1024$ pixels (of effective size $67.5 \mathrm{~nm}$ ) in $(\mathrm{X}, \mathrm{Y})$ and 61-121 steps (of $100 \mathrm{~nm}$ ) along the $\mathrm{Z}$ direction. The (per experiment uniform) lag time per Z-stack ranges between 1.7 and $2.7 \mathrm{~s}$. Addressed lag times are in the range where diffusive motion starts to exceed noise levels. The number of time points (i.e., Z-stacks) ranges between 92-168 per sample, and the duration of the experiments is up to $408 \mathrm{~s}$.

\subsubsection{Extracting Rotation and Translation}

For optimal visualization, the probes are dispersed in refractive index matching solvent mixtures $(n=1.45)$. Solvents are water-glycerol $\left(S_{1}, 1: 4\right.$ by weight, $\eta_{2}=59$ $\mathrm{mPa} . \mathrm{s})$ and water-glycerol-Dimethyl sulfoxide (DMSO) $\left(\mathrm{S}_{2}, 2: 4: 3\right.$ by wt., $\eta_{2}=20$ $\mathrm{mPa}$.s). Particle volume fractions are chosen around $0.3 \%$ to approach the dilute limit while keeping enough particles in the image volume for obtaining sufficient 
accuracy. Confocal Scanning Laser Microscopy (CSLM) in fluorescence mode is used to visualize only the labeled berries. Their Cartesian coordinates are extracted using well-known particle locating algorithms [51, 52]. The located berries are then grouped in clusters to identify which raspberry they belong to; this is achieved using a maximum distance criterion (see Appendix for details). Only raspberries that contain four or more non-coplanar tracers are kept. This minimum number is required for simultaneously finding the center location $(\mathrm{x}, \mathrm{y}, \mathrm{z})$ and optical radius $\left(\mathrm{R}_{\mathrm{fit}}\right)$ of the raspberry particle, which is achieved via leastsquares fitting to a sphere. Each obtained center location then provides an origin in a 3D Cartesian coordinate system that allows defining the spatial orientation of the raspberry probe based on the angular positions of the tracer berries. In this scheme, the translation of each raspberry is extracted from the time-dependent center location, leaving the rotational motion to be measured from the changes in orientation via applying rigid body transformations in between consecutive time steps [37]. The key steps involved in dissecting translational and rotational motion are illustrated in Figure 3.2. We have used a modified algorithm, based on ref [37], to calculate angular displacements from a rotational transformation matrix in terms of 3 Euler angles.

$$
\left[\begin{array}{l}
x_{i+1} \\
y_{i+1} \\
z_{i+1}
\end{array}\right]=R\left[\begin{array}{l}
x_{i} \\
y_{i} \\
z_{i}
\end{array}\right]
$$

Where $[\mathrm{x}, \mathrm{y}, \mathrm{z}]$ coordinates denote the location of an individual berry tracer at $\mathrm{i}^{\text {th }}$ time step, and $\mathrm{R}$ is the rotational transformation matrix. The calculation of $\mathrm{R}$ is as given in [37]. After obtaining this transformation matrix, we calculate the rotations around each principle axes. A general rotational matrix has the form:

$$
\mathrm{R}=\left[\begin{array}{ccc}
\cos \beta \cos \gamma & \sin \alpha \sin \beta \cos \gamma-\cos \alpha \sin \gamma & \cos \alpha \sin \beta \cos \gamma+\sin \alpha \sin \gamma \\
\cos \beta \sin \gamma & \sin \alpha \sin \beta \sin \gamma+\cos \alpha \cos \gamma & \cos \alpha \sin \beta \sin \gamma-\sin \alpha \cos \gamma \\
-\sin \beta & \sin \alpha \cos \beta & \cos \alpha \cos \beta
\end{array}\right]
$$

Where $\alpha, \beta$ and $\gamma$ are the rotations around the principal $\mathrm{x}, \mathrm{y}$ and $\mathrm{z}$ axes, respectively. This rotation matrix is used to calculate the Euler angles directly [53] (details are given in Appendix). Due to the bounded nature of rotation angles, the calculation of angular displacements relative to the axis of rotation will yield a diffusion coefficient greater than a factor of $3 / 2$ of the actual value [37]. For that reason, MSAD values are multiplied for a factor of $2 / 3$ before calculating the rotational diffusion coefficient using eq. (3.4). 
Construction of trajectories from the time-dependent coordinates is achieved via publicly available tracking routines [54]. The accuracy of the codes was tested with simulated data (mimicking typical experimental conditions) and gave good agreement (Figure A3, Appendix).

a)

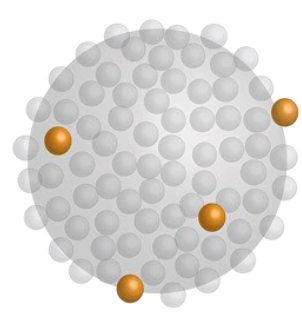

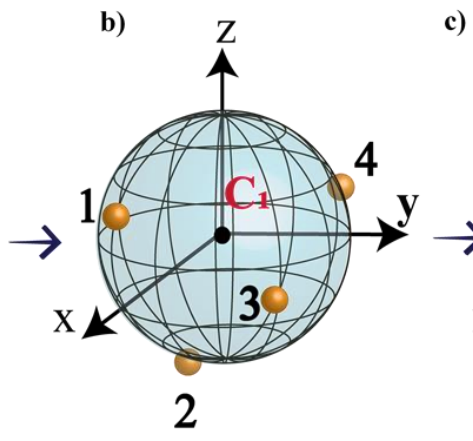

c)

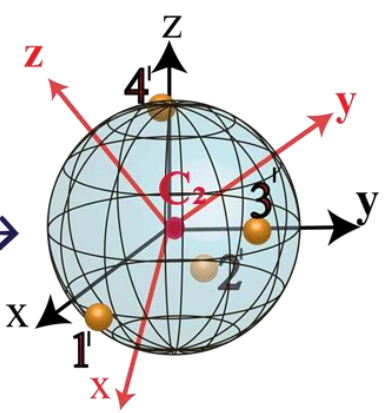

Figure 3.2. Key steps in measuring translation and rotation of a raspberry probe: a) 3D localization of the fluorescent berries, b) fitting a sphere encompassing the cluster, c) tracking the rotational displacement using a frame of reference fitted at the core center.

The coupled displacement of a group of berries on a raspberry is illustrated in Figure 3.3.a for several time steps. Typical rotational and translational trajectories for a raspberry are shown in Figures 3.3.b and 3.3.c. 

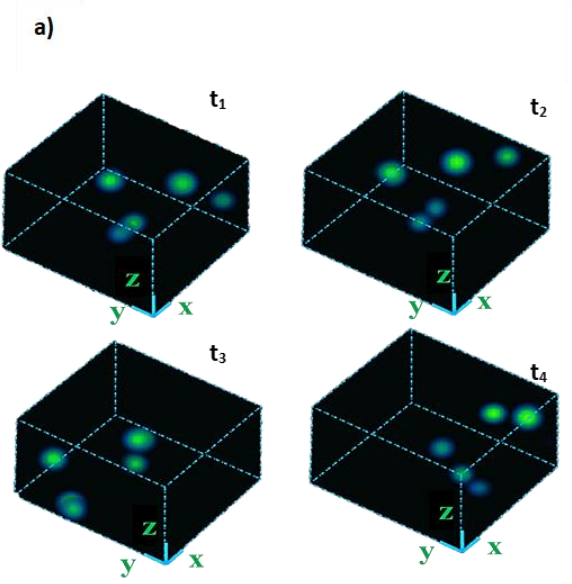

b)

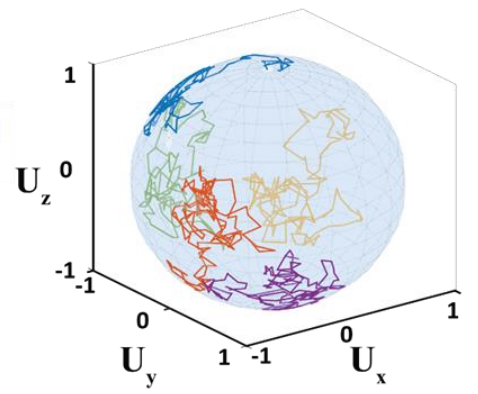

c)

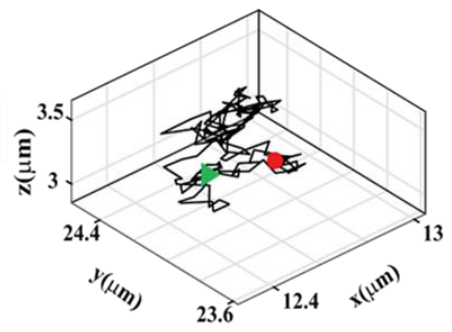

Figure 3.3. Visual demonstration of typical results for tracking a single raspberry probe. a) Coupled motion of fluorescent berry particles forming a cluster at different time steps (3D rendering done with ImageJ FIJI), b) Orientational trajectories of the five berries (differing in color) projected onto the surface of a unit sphere, c) Center-of-mass trajectory of a single raspberry particle (green=begin, red=end),

The mean squared displacement (MSD) versus lag time is calculated from the translational trajectories after the usual drift correction based on the ensembleaveraged motion of the tracked particles. Hereafter the translational diffusion coefficient $\mathrm{D}_{\mathrm{t}}$ is calculated from:

$$
\operatorname{MSD}=6 \mathrm{D}_{\mathrm{t}} \Delta \tau
$$

The mean squared angular displacement (MSAD) is calculated analogously, but without the need for a drift correction. The rotational diffusion coefficient $D_{r}$ is obtained from equation (4) below:

$$
\operatorname{MSAD}=4 \mathrm{D}_{\mathrm{r}} \Delta \tau
$$




\subsubsection{Estimation of Noise Levels}

The uncertainty in localization is estimated by tracking the 3D locations of fluorescent polystyrene particles $(\mathrm{r}=0.5 \mu \mathrm{m})$ immobilized on the bottom of the cuvettes. The measured (apparent) displacements are given in Appendix Figure A4.a. The corresponding MSDs are resulted in a noise level of $\sim 2.5 \times 10-3 \mu \mathrm{m}^{2}$ (given in Figure A4.b). The noise floor of the rotational tracking is estimated based on simulated trajectories of non-diffusing probes with four fluorescent tracers. Localization errors (taken as $\left[\begin{array}{lll}10 & 10 & 50\end{array}\right] \mathrm{nm}$ for [x y z] coordinates) are introduced in the simulation to mimic the real experimental conditions. The corresponding MSADs are resulted in a noise level of 2x10-3 $\mathrm{rad}^{2}$ (given in Figure A4.c). The resolution of the confocal microscope along the 3 principal axes is estimated by measuring point spread functions along $\mathrm{x}, \mathrm{y}$ and $\mathrm{z}$ axes. Corresponding resolutions are $\sim 270 \mathrm{~nm}$ for $\mathrm{x}$ and $\mathrm{y}$ and $445 \mathrm{~nm}$ for $\mathrm{z}$.

\subsection{Results}

\subsubsection{Validation of Brownian behavior}

We now examine the accuracy of measuring the diffusion coefficients for our raspberry probes in different stages of the data analysis. First, we consider the fitted sphere's radius $\left(\mathrm{R}_{\mathrm{fit}}\right)$ as extracted from the center locations of its berries. Given the dispersities of the core and berry systems, $R_{\text {fit }}$ should be close the sum of the typical radii: $\mathrm{R}_{\mathrm{fit}} \approx\left\langle\mathrm{R}_{\text {core }}\right\rangle+\left\langle\mathrm{R}_{\text {berry }}\right\rangle$, where the brackets indicate an average. Using transmission electron microscopy (TEM), we find $R_{\text {core }}=1041 \pm 33 \mathrm{~nm}$ and $R_{\text {berry }}=160 \pm 14 \mathrm{~nm}$. Figure 3.4. a shows $R_{\text {fit }}$ to peak at $\approx 1280 \mathrm{~nm}$, giving a fairly close correspondence. The calculated standard deviation of $35 \mathrm{~nm}$ is the resultant of the two polydispersities and the typical uncertainty in $\mathrm{R}_{\mathrm{fit}}$, which is estimated to be $44 \mathrm{~nm}$. The larger $R_{\text {fit }}$ values $(>1.3 \mu \mathrm{m})$ are rare and ascribed to photo-bleaching (after long exposure), which compromises the berry localizations.

The drift removal is an essential correction for the translational trajectories, unless the lag time $\tau<<\mathrm{D}_{t} / \mathrm{v}^{2}$, where $\mathrm{v}$ is the drift velocity [55]. In our case, drift analysis also contributes to validation because the vertical motion is dominated by sedimentation. The latter is illustrated in Figure 3.4.b for a typical probe trajectory. Accordingly, the axial displacement of the ensemble of raspberries shows good linearity, as can be seen in Figure 3.4.c. Since the particle volume fraction is very low, a comparison can be made between $v$ and theoretical Stokes 
sedimentation velocity of a smooth sphere. The dashed line in Figure 3.4.c is a linear fit that encompasses the axial drift displacement signal. Based on the Stokes Law, the effective radius of the corresponding smooth sphere calculated from the linear fit is $1.28 \mu \mathrm{m}$ which is close to the average TEM radius of the RP. Drift signals in the horizontal directions are ascribed to the microscope translation stage.

The principal results of this work, obtained after all analysis steps and thus accumulating the inaccuracies of all these steps, are shown in Figures $4 \mathrm{~d}$ and $4 \mathrm{e}$. Here, the solid black lines represent the (drift corrected) MSD and the MSAD, respectively. Both functions show a linear dependence on lag time, as expected for pure Brownian motion. The shortest addressed lag time is $2.4 \mathrm{~s}$, as determined by the acquisition time of a 3D confocal scan and the experiment duration is 387 s. Both the MSD and the MSAD are significantly above their respective noise floors.

Because of the spherical symmetry of the system, none of the two diffusion coefficients should show any directionality. Decompositions of the MSD and MSAD along the $\mathrm{X}, \mathrm{Y}$ and $\mathrm{Z}$ directions of the lab frame confirm this: the components along the different directions superimpose well in both Figure 3.4.d and Figure 3.4.e. Also the displacement histograms for a representative lag time (see insets) show a very good overlap and a Gaussian shape. This further corroborates that both types of diffusive motion are captured well. 
a)

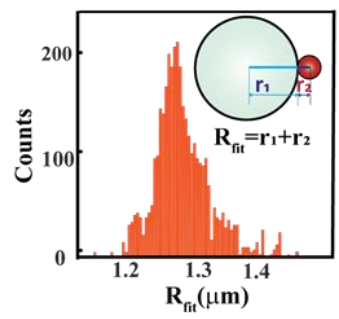

b)

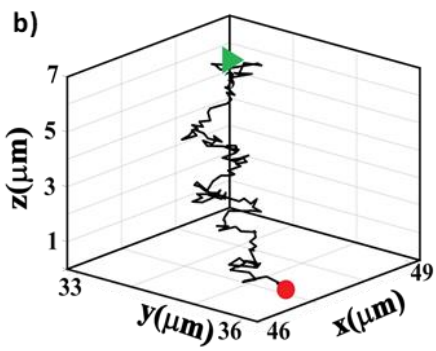

c)

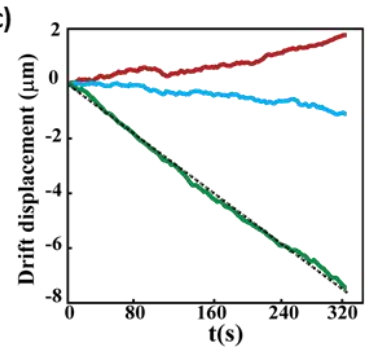

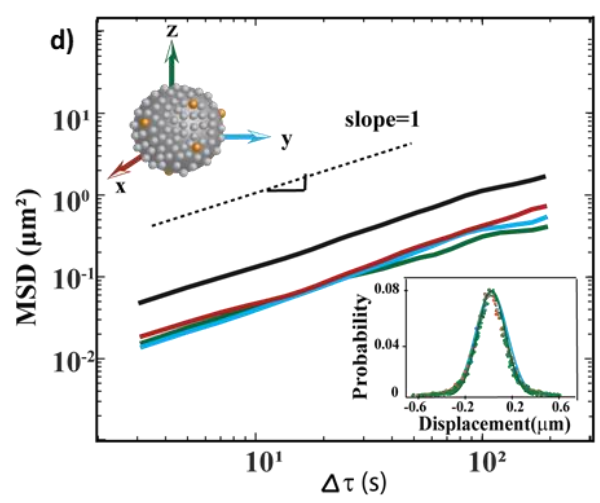

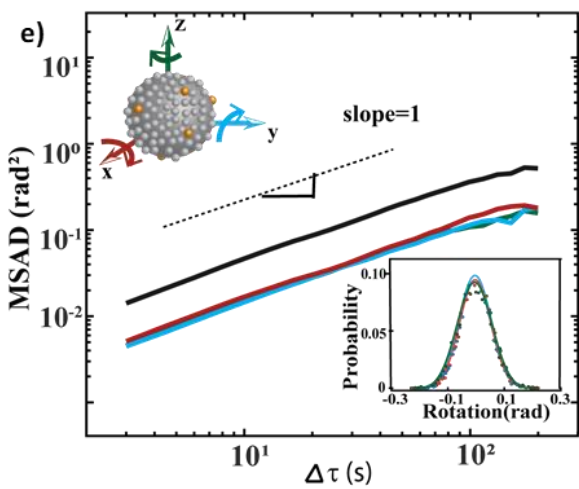

Figure 3.4. Key results of translational and rotational tracking. a) Histogram of fitted radius $R_{\text {fit }}$ (see inset) for both type of probes (which are optically identical). b) Typical 3D trajectory of a RP, before the removal of drift motion. c) Drift displacements for an ensemble of RP raspberries in solvent $\mathrm{S}_{1}$, along the $\mathrm{x}, \mathrm{y}$ and $\mathrm{z}$ directions. $\mathrm{d}$ ) MSD and e) MSAD versus lag time (solid black lines) for RP probes in solvent $\mathrm{S}_{1}$. Both functions are obtained from the same image data and based on 18 probes. Insets show decompositions along $\mathrm{x}, \mathrm{y}$ and $\mathrm{z}$ for the $\mathrm{MS}(\mathrm{A}) \mathrm{D}$, and for the displacement histograms. Color coding: red $=x$, blue $=y$, green $=z$. 


\subsubsection{Rotational and translational diffusion coefficients}

To assess the accuracy of the measured diffusion coefficients, we compare MSDs and MSADs for the two probe systems (RP, SP) in solvents with different viscosities: $\mathrm{S}_{1}(59 \pm 3 \mathrm{mPa} . \mathrm{s})$ and $\mathrm{S}_{2}(20 \pm 1 \mathrm{mPa} . \mathrm{s})$. Both probes are synthesized using the same core and berry particles, but for the SP system, a thicker silica layer has been overgrown to achieve a smoothened surface (Figure 3.1.e). This thicker layer also gives a significant increase in the final probe size (Figure 3.5.a). The MSAD measurements are shown in Figure 3.5.b. Regardless of the solvent, both the RP and SP probes demonstrate a purely diffusive behavior, i.e., a linear increase in MSAD with lag time. Extracted numerical data are summarized in Table 1, where the diffusion coefficients are obtained from linear fits for lag times up to $10 \mathrm{~s}$.
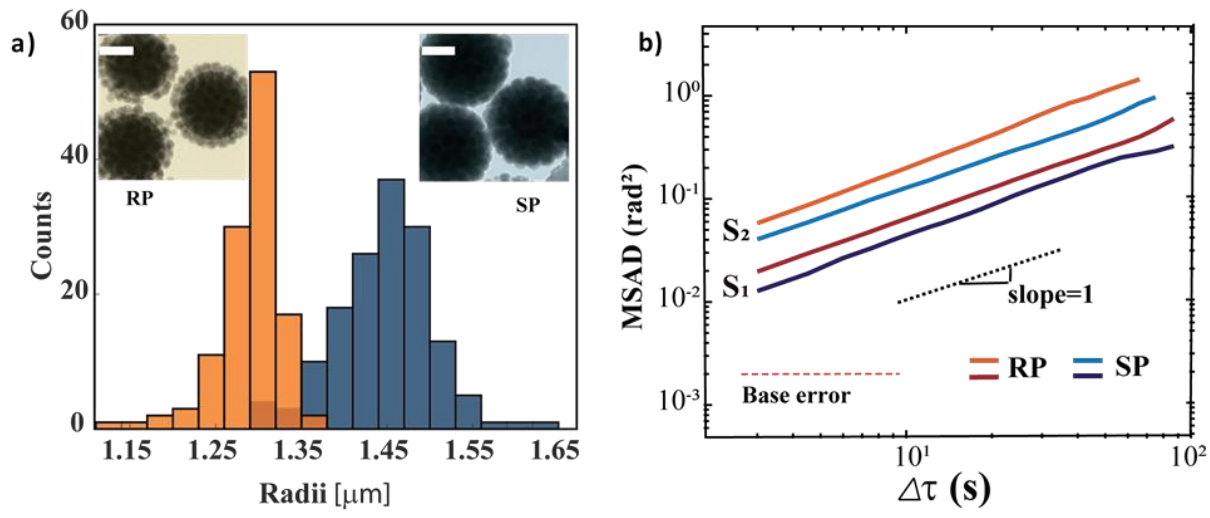

Figure 3.5. a) Size distributions and TEM close-ups for RP (orange) and SP (blue). Scale bar is $1 \mu \mathrm{m}$. b) MSAD versus lag time for raspberry systems RP (orange/brown) and SP (blue/purple) dispersed in solvents $S_{1}(\eta=59 \mathrm{mPa} . \mathrm{s})$ and in $\mathrm{S}_{2}(\eta=20 \mathrm{mPa} . \mathrm{s})$. 
Table 3.1. Overview of measured diffusion coefficients and some derived quantities. $R_{h}$ is the hydrodynamic radius of the equivalent smooth sphere.

\begin{tabular}{l|ccccc} 
& $\mathrm{D}_{\mathrm{t}}\left(\mu \mathrm{m}^{2} / \mathrm{s}\right)$ & $\mathrm{D}_{\mathrm{r}}\left(\mathrm{s}^{-1}\right)$ & $\mathrm{D}_{\mathrm{t}} / \mathrm{D}_{\mathrm{r}}\left(\mu \mathrm{m}^{2}\right)$ & $\mathrm{R}_{\mathrm{h}_{\_}}\left[\mathrm{D}_{\mathrm{t}}\right](\mu \mathrm{m})$ & $\mathrm{R}_{\mathrm{h}_{\_}}\left[\mathrm{D}_{\mathrm{r}}\right](\mu \mathrm{m})$ \\
\hline $\mathrm{RP}$ in $\mathrm{S}_{1}$ & $2.7810^{-3}$ & $1.5810^{-3}$ & 1.76 & 1.29 & 1.20 \\
$\mathrm{RP}$ in $\mathrm{S}_{2}$ & $9.7310^{-3}$ & $5.1810^{-3}$ & 1.88 & 1.09 & 1.15 \\
& & & & & \\
$\mathrm{SP}$ in $\mathrm{S}_{1}$ & $2.2310^{-3}$ & $1.1310^{-3}$ & 1.97 & 1.61 & 1.34 \\
$\mathrm{SP}$ in $\mathrm{S}_{2}$ & $7.5810^{-3}$ & $3.1010^{-3}$ & 2.45 & 1.40 & 1.37
\end{tabular}

In the dilute limit, it is interesting to compare the measured $D_{r}$ and $D_{t}$ values to the theoretical expressions for smooth spheres in a Newtonian liquid with a noslip boundary condition [56]. $D_{t}$ is given by Stokes-Einstein equation, and $D_{r}$ is given by Stokes-Einstein-Debye equation as follows:

$$
\begin{aligned}
D_{t} & =\frac{k_{b} T}{6 \Pi \eta R} \\
D_{r} & =\frac{k_{b} T}{8 \Pi \eta R^{3}}
\end{aligned}
$$

where $\mathrm{k}_{\mathrm{b}}$ is the Boltzmann constant, $\mathrm{T}$ is temperature, and $\mathrm{R}$ is the radius. Also, for rough spheres, $D_{r}$ and $D_{t}$ should be inversely proportional to the solvent viscosity $\eta$. Calculating $\mathrm{D}_{\mathrm{i}}\left(\mathrm{S}_{1}\right) / \mathrm{D}_{\mathrm{i}}\left(\mathrm{S}_{2}\right)$ with $\mathrm{i} \in \mathrm{r}$, t for RP and SP separately, we obtain $\eta\left(\mathrm{S}_{1}\right) / \eta\left(\mathrm{S}_{2}\right)=3.2 \pm 0.3$, in good agreement with the $3.0 \pm 0.2$ obtained from the measured viscosities.

Comparing the cases where the same probe system is dispersed in 2 different solvents, a different accuracy assessment can be made by calculating $D_{t} / D_{r}$. Now the viscosity effect is 'divided out' because of the inverse proportionality of both $D_{t}$ and $D_{r}$ to $\eta$. The results in Table 3.1 show very similar ratios $(\sim 1.82 \pm 0.08$ $\left.\mu \mathrm{m}^{2}\right)$ for RP and more differing ones $\left(\sim 2.2 \pm 0.3 \mu \mathrm{m}^{2}\right)$ for SP. The larger $\mathrm{D}_{t} / \mathrm{D}_{\mathrm{r}}$ values for the SP probes are not unexpected, considering the $\mathrm{R}^{2}$ proportionality for smooth spheres. The small variations among the measurements in different solvents might be due to a 'sampling effect': a combined effect of the polydispersity (Figure 3.5.a) and the finite number of probes (typically 20-30) in a single image volume.

Lastly, we use the standard expressions (eqs. 3.5 and 3.6) for $D_{t}$ and $D_{r}$ of smooth spheres in the dilute limit to calculate effective radii $R_{h}$ from the measured 64 
diffusion coefficients and solvent viscosities. The $R_{h}$ values in Table 3.1 are comparable to those from the TEM measurements (Figure 3.5.a). Some slight differences between the values obtained from $S_{1}$ and $S_{2}$ are found; these can be attributed to the fact that each of the four raspberry/solvent combinations was explored with a fresh solvent mixture (possibility of slight differences in viscosities) and a limited number of particles (possibly introducing a sampling effect). A striking observation is that for both $R P$ and $S P$, the $R_{h}$ values obtained from $D_{r}$ correspond better with TEM measurements. Considering the $R^{3}$ proportionality of $D_{r}$ for smooth spheres (while it is $\sim R^{-1}$ for $D_{t}$ ), it is likely that $D_{r}$ provides a more precise measure for $R_{h}$. Considering how close $\left\langle R_{h}\left[D_{t}\right]>\right.$ and $\left\langle\mathrm{R}_{\mathrm{h}}\left[\mathrm{D}_{\mathrm{r}}\right]\right\rangle$ are to those of the TEM measurements, we conclude that the surface roughness (being larger for RP) does not have a discernable effect on either of the two diffusion coefficients in the dilute limit.

\subsection{Application Scope}

Our novel probes could be useful in various cases where simultaneous 3D tracking of individual particle locations and orientations is needed. Due to the tunability of the outer layer thickness, surface roughness can be altered. This offers a broad application potential for our particles in many colloidal dynamics studies. At low particle concentrations, the effects of roughness on the two diffusion coefficients were too subtle to be measured. However, rough probes can be employed at high concentrations to study the relation between roughness and diffusion. In the dense regime, particle-particle interactions play an important role in colloidal dynamics, and strong correlations between roughness and jamming have already been found [57-59]. In the smooth limit, our probes can be used for shedding more light on the effect of colloidal interactions on rotational diffusion $[60,61]$ or transient phenomena like glassy dynamics and crystallization. Also, in complex fluids whose structure is not dictated by particles, our (rough or smooth) probes could provide information about local (mechanical) properties. Here the surrounding 'bulk' material could, e.g., be polymer solutions/gels or liquid interfaces.

Lastly, the synthesis and utilization of raspberry probes are not limited to the demonstrated methodology. Due to the simple synthesis and the use of only one fluorescent label for simultaneous tracking of two different motions, the 'berry platform' can pave the way for designing similar probes with additional shape isotropy. To make use of our method, the minimum number of tracer berries is 
four, but the systems with a higher number of fluorescent tracers will also provide accurate results. One design criterion to keep in mind here is that fluorescent tracers' distances must be larger than the optical resolution. Applying the 'labeled raspberry' concept while using different materials is yet another direction. Probes could also be functionalized as active colloids, soft compressible particles, or serve in biomimicking studies to resolve dynamics of biological processes [19, $62]$.

\subsection{Conclusions}

The use of (confocal) microscopy to track the translational motion of colloidal spheres has evolved into a broadly applied method in soft matter science in the past two decades. Tracking the rotation of the spheres is much less common, despite the enhanced insights it can offer. This could be related to limitations of existing probes regarding their (non-spherical) shape or (non-uniform) surface chemistry. We developed very-nearly-spherical ('raspberry') probes with an allsilica surface. Surface roughness can be controlled via the thickness of the overgrown silica layer. Adding 4-6 fluorescent tracers in the shell enables precise measurement of both the center location and the probe's orientation. We demonstrated the utility of our probes by measuring 3D translational and rotational diffusion coefficients in different solvents. In the dilute limit, all diffusion coefficients turned out to be similar to theoretical values for smooth spheres of the same size. We envision that the concept of 'raspberry '-based rotational-translational probes can be exploited in different directions, for example, the use of different materials, particularly the study of more complex systems with heterogeneous dynamics.

\section{Acknowledgments}

This work was financially supported by NWO-CW (ECHO grant 712.016.004). We thank Mark Smithers for SEM imaging, Rico Keim for TEM imaging, Joris Sprakel for providing de-drifting Matlab routines, and Dirk van den Ende for fruitful discussions. 


\section{Bibliography}

[1] T.G. Mason, K. Ganesan, J.H. vanZanten, D. Wirtz, S.C. Kuo, Particle tracking microrheology of complex fluids, Physical Review Letters, 79 (1997) 3282-3285.

[2] D.T. Chen, E.R. Weeks, J.C. Crocker, M.F. Islam, R. Verma, J. Gruber, A.J. Levine, T.C. Lubensky, A.G. Yodh, Rheological microscopy: Local mechanical properties from microrheology, Physical Review Letters, 90 (2003).

[3] T.A. Waigh, Microrheology of complex fluids, Reports on Progress in Physics, 68 (2005) 685-742.

[4] V. Breedveld, D.J. Pine, Microrheology as a tool for high-throughput screening, Journal of Materials Science, 38 (2003) 4461-4470.

[5] A. Yethiraj, A. van Blaaderen, A colloidal model system with an interaction tunable from hard sphere to soft and dipolar, Nature, 421 (2003) 513-517.

[6] W.K. Kegel, A. van Blaaderen, Direct observation of dynamical heterogeneities in colloidal hard-sphere suspensions, Science, 287 (2000) 290293.

[7] E.R. Weeks, J.C. Crocker, A.C. Levitt, A. Schofield, D.A. Weitz, Threedimensional direct imaging of structural relaxation near the colloidal glass transition, Science, 287 (2000) 627-631.

[8] P.N. Pusey, W. van Megen, Observation of a glass transition in suspensions of spherical colloidal particles, Phys Rev Lett, 59 (1987) 2083-2086.

[9] E.R. Weeks, D.A. Weitz, Properties of cage rearrangements observed near the colloidal glass transition, Phys Rev Lett, 89 (2002) 095704.

[10] M.D. Wehrman, A. Leduc, H.E. Callahan, M.S. Mazzeo, M. Schumm, K.M. Schultz, Rheological Properties and Structure of Step- and Chain-Growth Gels Concentrated above the Overlap Concentration, Aiche J., 64 (2018) 3168-3176.

[11] K.M. Schultz, A.D. Baldwin, K.L. Kiick, E.M. Furst, Gelation of Covalently Cross-Linked PEG-Heparin Hydrogels, Macromolecules, 42 (2009) 5310-5316. 
[12] C.R. Nugent, K.V. Edmond, H.N. Patel, E.R. Weeks, Colloidal glass transition observed in confinement, Physical Review Letters, 99 (2007).

[13] H.B. Eral, J.M. Oh, D. van den Ende, F. Mugele, M.H.G. Duits, Anisotropic and Hindered Diffusion of Colloidal Particles in a Closed Cylinder, Langmuir, 26 (2010) 16722-16729.

[14] H.B. Eral, D. van den Ende, F. Mugele, M.H.G. Duits, Influence of confinement by smooth and rough walls on particle dynamics in dense hardsphere suspensions, Physical Review E, 80 (2009).

[15] S. Ghosh, D. Wijnperle, F. Mugele, M.H.G. Duits, Dynamics of colloids confined in microcylinders, Soft matter, 12 (2016) 1621-1630.

[16] T. Moschakis, Microrheology and particle tracking in food gels and emulsions, Current Opinion in Colloid \& Interface Science, 18 (2013) 311-323.

[17] M.H.G. Duits, Y.X. Li, S.A. Vanapalli, F. Mugele, Mapping of spatiotemporal heterogeneous particle dynamics in living cells, Physical Review E, 79 (2009).

[18] J.C. Crocker, B.D. Hoffman, Multiple-particle tracking and two-point microrheology in cells, Cell Mechanics2007, pp. 141-178.

[19] S.-L. Liu, Z.-L. Zhang, Z.-Q. Tian, H.-S. Zhao, H. Liu, E.-Z. Sun, G.F. Xiao, W. Zhang, H.-Z. Wang, D.-W. Pang, Effectively and efficiently dissecting the infection of influenza virus by quantum-dot-based single-particle tracking, ACS nano, 6 (2012) 141-150.

[20] S.M. Anthony, Y. Yu, Tracking single particle rotation: probing dynamics in four dimensions, Analytical Methods, 7 (2015) 7020-7028.

[21] M. Kim, S.M. Anthony, S.C. Bae, S. Granick, Colloidal rotation near the colloidal glass transition, The Journal of chemical physics, 135 (2011) 054905.

[22] M.D. Ediger, Spatially heterogeneous dynamics in supercooled liquids, Annual review of physical chemistry, 51 (2000) 99-128.

[23] S. Schütter, J. Roller, A. Kick, J.-M. Meijer, A. Zumbusch, Real-space imaging of translational and rotational dynamics of hard spheres from the fluid to the crystal, Soft matter, 13 (2017) 8240-8249. 
[24] G. Boniello, C. Blanc, D. Fedorenko, M. Medfai, N.B. Mbarek, M. In, M. Gross, A. Stocco, M. Nobili, Brownian diffusion of a partially wetted colloid, Nature materials, 14 (2015) 908-911.

[25] A. Stocco, B. Chollet, X. Wang, C. Blanc, M. Nobili, Rotational diffusion of partially wetted colloids at fluid interfaces, Journal of colloid and interface science, 542 (2019) 363-369.

[26] S.W. Chee, U. Anand, G. Bisht, S.F. Tan, U. Mirsaidov, Direct Observations of the Rotation and Translation of Anisotropic Nanoparticles Adsorbed at a Liquid-Solid Interface, Nano letters, 19 (2019) 2871-2878.

[27] P. Kukura, H. Ewers, C. Müller, A. Renn, A. Helenius, V. Sandoghdar, Highspeed nanoscopic tracking of the position and orientation of a single virus, Nature methods, 6 (2009) 923.

[28] B.W.M. Kuipers, M.C.A. van de Ven, R.J. Baars, A.P. Philipse, Simultaneous measurement of rotational and translational diffusion of anisotropic colloids with a new integrated setup for fluorescence recovery after photobleaching, Journal of Physics-Condensed Matter, 24 (2012).

[29] M.P. Lettinga, G.H. Koenderink, B.W.M. Kuipers, E. Bessels, A.P. Philipse, Rotational dynamics of colloidal spheres probed with fluorescence recovery after photobleaching, Journal of Chemical Physics, 120 (2004) 4517-4529.

[30] G.H. Koenderink, A.P. Philipse, Rotational and translational self-diffusion in colloidal sphere suspensions and the applicability of generalized StokesEinstein relations, Langmuir, 16 (2000) 5631-5638.

[31] Y. Wang, C. Li, G.J. Pielak, Effects of proteins on protein diffusion, Journal of the American Chemical Society, 132 (2010) 9392-9397.

[32] H. Sun, Z. Wang, Y. He, Direct Observation of Spatiotemporal Heterogeneous Gelation by Rotational Tracking of a Single Anisotropic Nanoprobe, ACS nano, 13 (2019) 11334-11342.

[33] Z. Cheng, T. Mason, Rotational diffusion microrheology, Physical review letters, 90 (2003) 018304.

[34] Y. Peng, L. Lai, Y.-S. Tai, K. Zhang, X. Xu, X. Cheng, Diffusion of ellipsoids in bacterial suspensions, Physical review letters, 116 (2016) 068303. 
[35] J. Roller, P. Pfleiderer, J.-M. Meijer, A. Zumbusch, Detection and tracking of anisotropic core-shell colloids, Journal of Physics: Condensed Matter, 30 (2018) 395903.

[36] M. Hoffmann, C.S. Wagner, L. Harnau, A. Wittemann, 3D Brownian diffusion of submicron-sized particle clusters, ACS nano, 3 (2009) 3326-3334.

[37] G.L. Hunter, K.V. Edmond, M.T. Elsesser, E.R. Weeks, Tracking rotational diffusion of colloidal clusters, Optics express, 19 (2011) 17189-17202.

[38] S.M. Anthony, M. Kim, S. Granick, Translation-rotation decoupling of colloidal clusters of various symmetries, The Journal of chemical physics, 129 (2008) 244701.

[39] T. Besseling, M. Hermes, A. Kuijk, B. De Nijs, T. Deng, M. Dijkstra, A. Imhof, A. Van Blaaderen, Determination of the positions and orientations of concentrated rod-like colloids from 3D microscopy data, Journal of Physics: Condensed Matter, 27 (2015) 194109.

[40] J.R. Gomez-Solano, A. Blokhuis, C. Bechinger, Dynamics of self-propelled Janus particles in viscoelastic fluids, Physical review letters, 116 (2016) 138301.

[41] S.M. Anthony, M. Kim, S. Granick, Single-particle tracking of janus colloids in close proximity, Langmuir, 24 (2008) 6557-6561.

[42] S. Jiang, Q. Chen, M. Tripathy, E. Luijten, K.S. Schweizer, S. Granick, Janus particle synthesis and assembly, Advanced materials, 22 (2010) 1060-1071.

[43] B. Liu, A. Böker, Measuring rotational diffusion of colloidal spheres with confocal microscopy, Soft matter, 12 (2016) 6033-6037.

[44] W. Stöber, A. Fink, E. Bohn, Controlled growth of monodisperse silica spheres in the micron size range, Journal of colloid and interface science, 26 (1968) 62-69.

[45] G. Bogush, M. Tracy, C. Zukoski Iv, Preparation of monodisperse silica particles: control of size and mass fraction, Journal of non-crystalline solids, 104 (1988) 95-106.

[46] M. Zanini, C.-P. Hsu, T. Magrini, E. Marini, L. Isa, Fabrication of rough colloids by heteroaggregation, Colloids and Surfaces A: Physicochemical and Engineering Aspects, 532 (2017) 116-124. 
[47] A. Van Blaaderen, J. Van Geest, A. Vrij, Monodisperse colloidal silica spheres from tetraalkoxysilanes: particle formation and growth mechanism, Journal of Colioid and Interface Science, 154 (1992) 481-501.

[48] S.d. Coenen, C. De Kruif, Synthesis and growth of colloidal silica particles, Journal of colloid and interface science, 124 (1988) 104-110.

[49] B. Ilhan, C. Annink, D. Nguyen, F. Mugele, I. Sîretanu, M.H. Duits, A method for reversible control over nano-roughness of colloidal particles, Colloids and surfaces A: Physicochemical and engineering aspects, 560 (2019) 50-58.

[50] S. Ghosh, F. Mugele, M.H. Duits, Effects of shear and walls on the diffusion of colloids in microchannels, Physical Review E, 91 (2015) 052305.

[51] Y. Gao, M.L. Kilfoi, Accurate detection and complete tracking of large populations of features in three dimensions, Optics express, 17 (2009) 4685-4704.

[52] K.E. Jensen, N. Nakamura, Note: An iterative algorithm to improve colloidal particle locating, Review of Scientific Instruments, 87 (2016) 066103.

[53] G.G. Slabaugh, Computing Euler angles from a rotation matrix, Retrieved on August, 6 (1999) 39-63.

[54] J.C. Crocker, E.R. Weeks, Particle tracking using IDL, Retreived from http://www. physics. emory. edu/faculty/weeks//idl, (2011).

[55] M.H. Duits, S. Ghosh, F. Mugele, Measuring advection and diffusion of colloids in shear flow, Langmuir, 31 (2015) 5689-5700.

[56] A.P. Philipse, Brownian Motion, Springer 2018.

[57] C.P. Hsu, S.N. Ramakrishna, M. Zanini, N.D. Spencer, L. Isa, Roughnessdependent tribology effects on discontinuous shear thickening, Proceedings of the National Academy of Sciences of the United States of America, 115 (2018) 5117-5122.

[58] L.C. Hsiao, S. Jamali, E. Glynos, P.F. Green, R.G. Larson, M.J. Solomon, Rheological State Diagrams for Rough Colloids in Shear Flow, Physical Review Letters, 119 (2017). 
[59] L.C. Hsiao, I. Saha-Dalal, R.G. Larson, M.J. Solomon, Translational and rotational dynamics in dense suspensions of smooth and rough colloids, Soft Matter, 13 (2017) 9229-9236.

[60] G.H. Koenderink, H.Y. Zhang, D. Aarts, M.P. Lettinga, A.P. Philipse, G. Nagele, On the validity of Stokes-Einstein-Debye relations for rotational diffusion in colloidal suspensions, Faraday Discussions, 123 (2003) 335-354.

[61] G.H. Koenderink, M.P. Lettinga, A.P. Philipse, Rotational dynamics of charged colloidal spheres: Role of particle interactions, Journal of Chemical Physics, 117 (2002) 7751-7764.

[62] K. Welsher, H. Yang, Multi-resolution 3D visualization of the early stages of cellular uptake of peptide-coated nanoparticles, Nature nanotechnology, 9 (2014) 198. 


\section{Appendix}

\section{Stability of colloidal systems:}

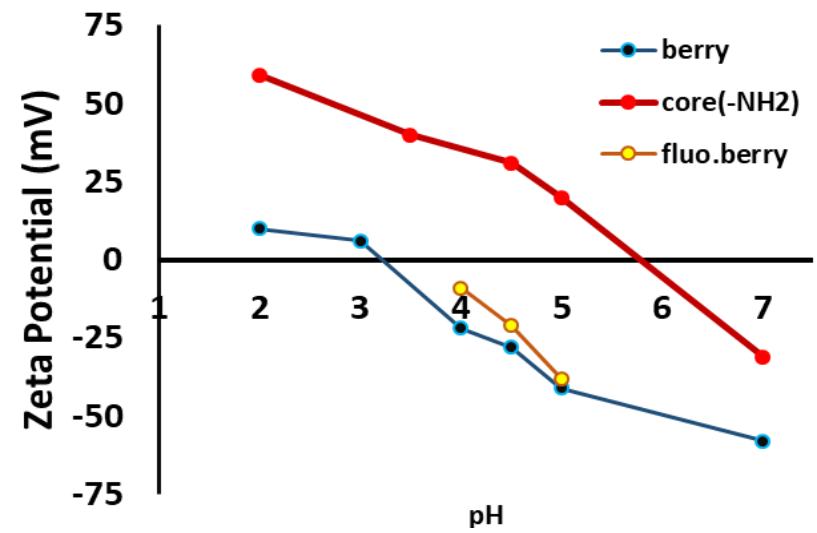

Figure A1. Zeta potential vs. $\mathrm{pH}$ for the core and berry particles measured in the aqueous phase ( $\mathrm{pH}$ adjustments are done with $\mathrm{HNO}_{3}$, Smoluchowski approximation)

\section{Size Distributions}

a)

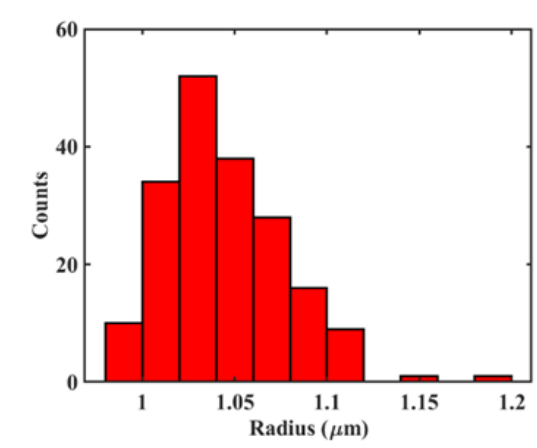

b)

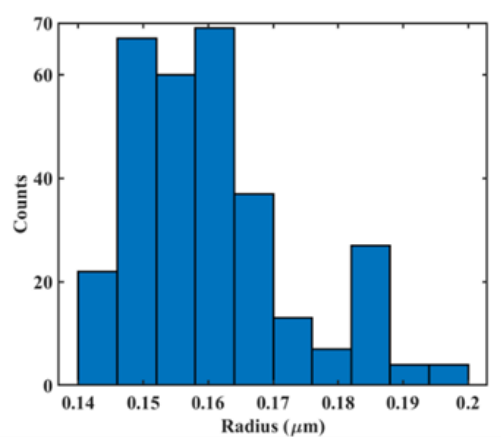

Figure A2. TEM size distributions of a) core and b) berry particles 


\section{Data Analysis Steps}

Clustering Analysis: Deciding which berry belong to which raspberry

After localizing the fluorescently labeled tracer berries in 3D at each time step, clustering analysis is done to identify individual raspberry particles. All tracer berries in the image volume occurring within a maximum distance $\Delta$ from each other are assigned to a specific cluster. Here $\Delta=2\left(\mathrm{R}_{\text {core }}+\mathrm{R}_{\text {berry }}+\delta\right)$, where $\delta$ takes into account the localization error. First, clusters containing less than four tracers are discarded due to the requirement of core center localization via sphere fitting. Second, the largest distance between two berries in the same cluster is calculated. If this distance is larger than $\Delta$, the cluster is discarded because it contains more than one raspberry core center. The latter situation can arise if two raspberry particles approach each other very closely (e.g., via Brownian motion). Since the criterion is applied for every time step, it means that the trajectories of the involved raspberries will be terminated (and rejuvenated after they moved apart over a sufficient distance).

\section{Analysis of Rotational Motion}

After the clustering analysis, a spherical fit [1] is applied to the tracer berry locations in a cluster at each time step. For each raspberry and time step, the fitted radius $\left(\mathrm{R}_{\mathrm{fit}}\right)$ should fall within the expected error margin of $\mathrm{R}_{\text {core }}+\mathrm{R}_{\text {berry. }}$. Failure to meet this criterion is taken as an indication for a bad data fit (e.g., nearly coplanar tracer berries), and the cluster is excluded from the analysis.

Next, the rotation is measured via a method that was slightly adapted from ref [2]. The key steps were already summarized in the main text. To subsequent extraction of the rotations around the principal $\mathrm{x}, \mathrm{y}, \mathrm{z}$ axes from the rotational transformation matrix of $\mathrm{R}$ works as follows:

The definition of rotation around each axis is defined as:

$$
\mathrm{R}_{\mathrm{x}}(\alpha)=\left[\begin{array}{rrr}
1 & 0 & 0 \\
0 & \cos \alpha & -\sin \alpha \\
0 & \sin \alpha & \cos \alpha
\end{array}\right]
$$




$$
\begin{aligned}
& \mathrm{R}_{\mathrm{y}}(\beta)=\left[\begin{array}{llr}
\cos \beta & 0 & \sin \beta \\
0 & 1 & 0 \\
-\sin \beta & 0 & \cos \beta
\end{array}\right] \\
& \mathrm{R}_{\mathrm{z}}(\gamma)=\left[\begin{array}{llr}
\cos \gamma & -\sin \gamma & 0 \\
0 & 1 & 0 \\
0 & 0 & 1
\end{array}\right]
\end{aligned}
$$

Consider $\mathrm{R}$ as a sequence of 3 rotations; first around $\mathrm{x}$, then $\mathrm{y}$ and $\mathrm{z}$ axis:

$$
\mathrm{R}=\mathrm{R}_{\mathrm{z}}(\gamma) \mathrm{R}_{\mathrm{y}}(\beta) \mathrm{R}_{\mathrm{x}}(\alpha)
$$

Equation (3.10) will result in:

$$
R=\left[\begin{array}{ccc}
\cos \beta \cos \gamma & \sin \alpha \sin \beta \cos \gamma-\cos \alpha \sin \gamma & \cos \alpha \sin \beta \cos \gamma+\sin \alpha \sin \gamma \\
\cos \beta \sin \gamma & \sin \alpha \sin \beta \sin \gamma+\cos \alpha \cos \gamma & \cos \alpha \sin \beta \sin \gamma-\sin \alpha \cos \gamma \\
-\sin \beta & \sin \alpha \cos \beta & \cos \alpha \cos \beta
\end{array}\right]
$$

For small rotations in between consecutive frames such that; $\alpha, \beta, \gamma \ll \pi / 2$ these angles can be calculated via [3]:

$$
\begin{gathered}
\beta=\sin ^{-1}\left(\mathrm{R}_{31}\right) \\
\alpha=\operatorname{atan} 2\left(\frac{\mathrm{R}_{32}}{\cos \beta}, \frac{\mathrm{R}_{33}}{\cos \beta}\right) \\
\gamma=\operatorname{atan} 2\left(\frac{\mathrm{R}_{21}}{\cos \beta}, \frac{\mathrm{R}_{11}}{\cos \beta}\right)
\end{gathered}
$$




\section{Simulating Data}

The accuracy of the rotational tracking algorithm has been checked by using simulated data that mimic experimental conditions. In a typical simulation, probe particles are placed in a 3D box of the same size as the experiments $(\sim 70 \times 70 \times 12$ $\mu \mathrm{m})$. The number of probes in the system is chosen in accordance with a dilute regime of $\sim \varphi<0.5 \%$.

A Gaussian distribution is applied for the number of tracer berries per core. The average number of berries is chosen to be 4 , with a standard deviation of 1 . These fluorescent tracers are kept at least one berry diameter apart so that they do not overlap. The sizes of core and berry particles are based on the experimental systems, leading to a total raspberry radius of $1.30 \mu \mathrm{m}$. For each time step (taken similar to that of experimental lag time), Gaussian random 3D displacements and $3 \mathrm{D}$ rotations are imposed on all probe particles in accordance with the respective theoretical diffusion coefficients for smooth spheres. After imposing the displacements, random Gaussian noise is added to all the berry locations to mimic the localization error $([10,10,50 \mathrm{~nm}]$ for $[\mathrm{x}, \mathrm{y}, \mathrm{z}])$ error of the berries by the CSLM system.

Periodic boundary conditions are applied for the probes moving out of the system box. All corresponding berry locations over time are saved as trajectory array. These trajectory arrays of the berries are then analyzed with the clustering algorithm described above. 


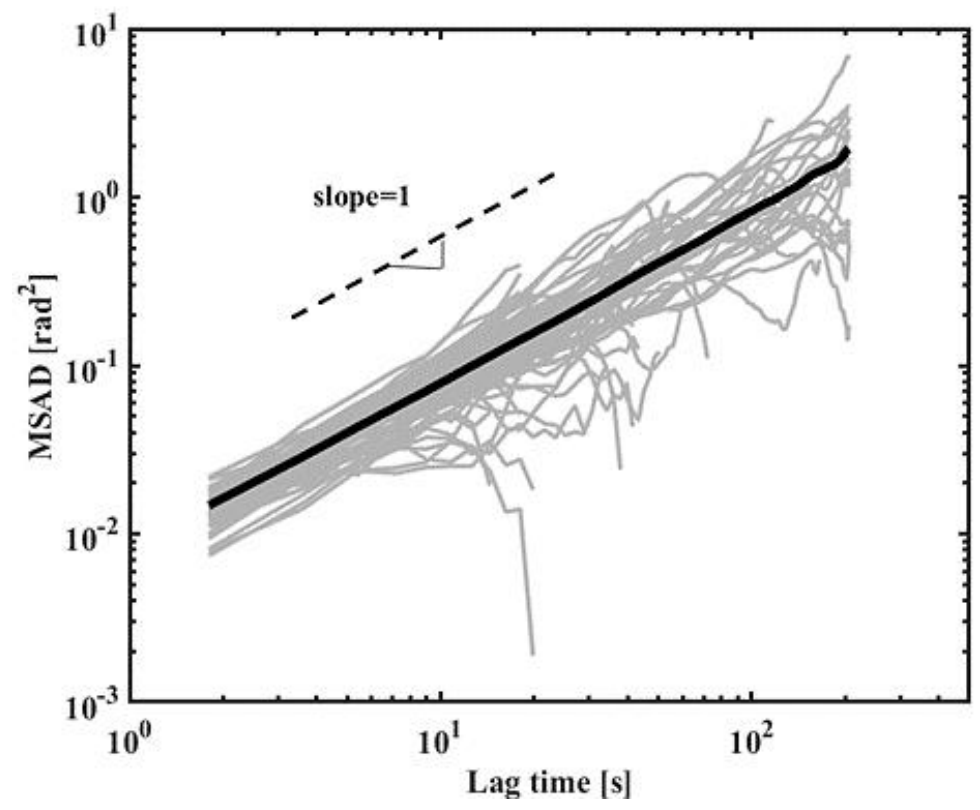

Figure A3. MSADs from simulations for 79 probes with input translational and rotational Brownian displacements according to the Stokes-Einstein and Stokes-Einstein-Debye relations for smooth spheres. Spheres with a radius of $1.30 \mu \mathrm{m}$ are dispersed in solvent $\mathrm{S} 1$ with a viscosity of $59 \mathrm{mPa} . \mathrm{s}$. The unit lag time is $1.8 \mathrm{sec}$. (grey=individual probes, black=mean value). 


\section{Estimations of 3D Localization Errors and Noise Levels for Translation and Rotation}

a)

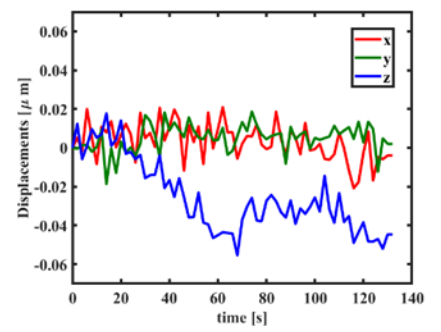

b)

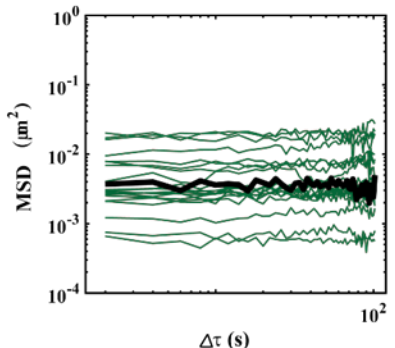

c)

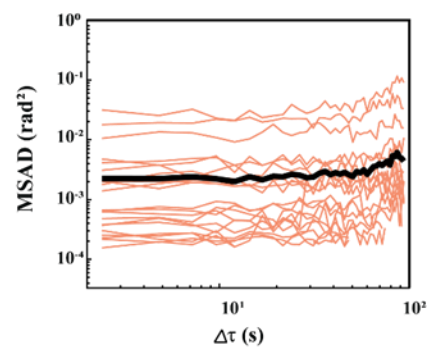

Figure A4. a) Measured displacements of immobilized particles over time. Noise floors for b) MSDs from immobilized particles (green: individual particles, black: average) c) MSADs obtained from simulations of non-diffusive raspberry probes (orange: individual particles, black=average. 


\section{Particle Surface Roughness}
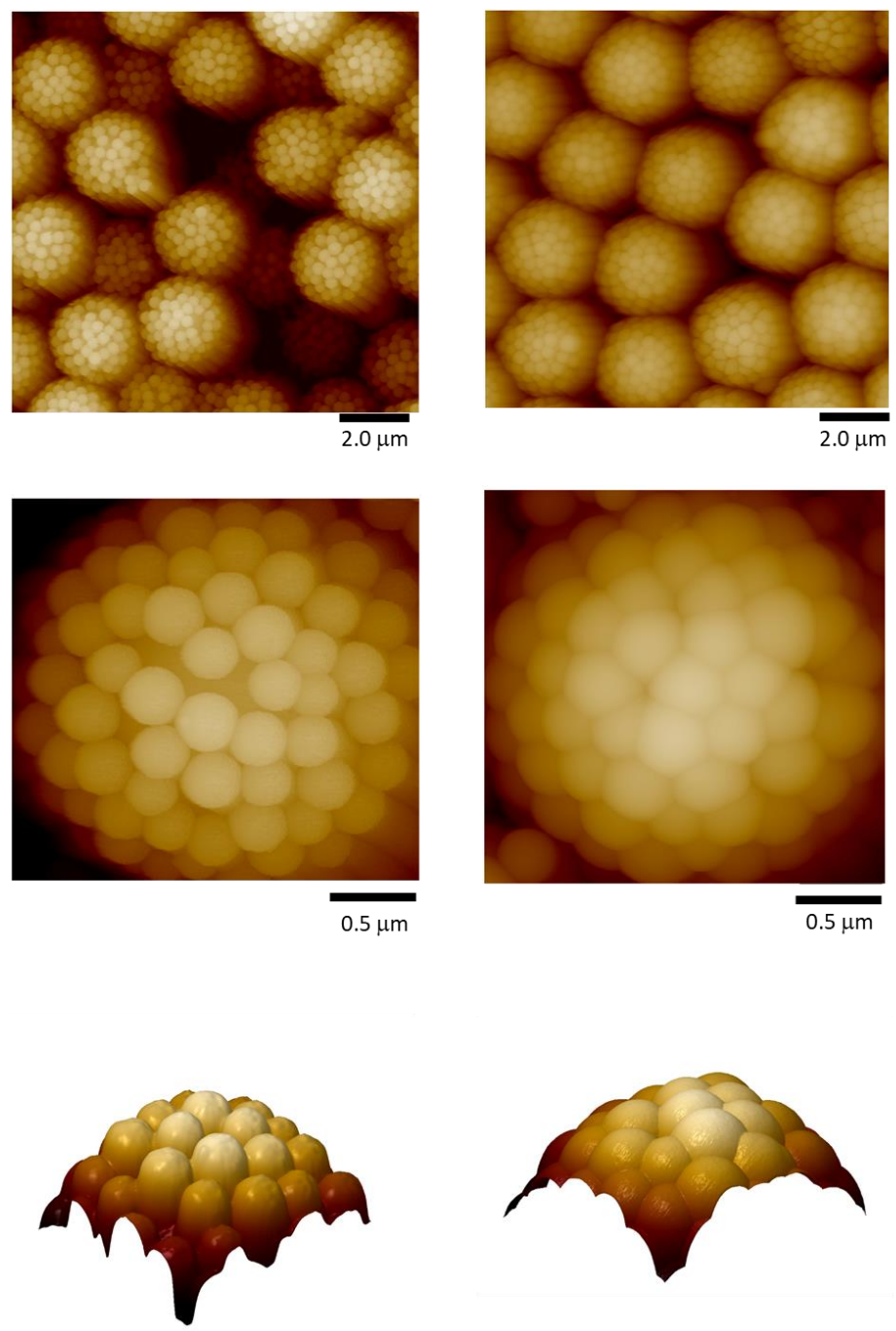

Figure A5. AFM images of RP and SP particles 


\section{Bibliography}

[1] A. Jennings, Sphere Fit (least squared), MathWorks.

[2] G.L. Hunter, K.V. Edmond, M.T. Elsesser, E.R. Weeks, Tracking rotational diffusion of colloidal clusters, Optics express, 19 (2011) 17189-17202.

[3] G.G. Slabaugh, Computing Euler angles from a rotation matrix, Retrieved on August, 6 (1999) 39-63. 


\section{4}

\section{Roughness Induced Rotational Slowdown near the Colloidal Glass Transition*}

Roughening the surface of spherical colloids can drastically change their translational and rotational dynamics in dense suspensions. Using 3D confocal microscopy, we show that roughness not only lowers the concentration of the translational colloidal glass transition but also generates a broad concentration range in which the rotational Brownian motion changes signature from highamplitude diffusive to low-amplitude rattling. This hitherto not reported second glass transition for rough spherical colloids emerges when the particle intersurface distance becomes comparable to the roughness length scale. Interlocking contacts are responsible for restricting the particle rotations.

*The research described in this chapter has been published as Ilhan, B., Mugele, F., \& Duits, M. H. (2021). Roughness Induced Rotational Slowdown near the Colloidal Glass Transition. Journal of Colloid and Interface Science, 607, 1709-1716 


\subsection{Introduction}

Glass transitions in molecular systems remain a highly intriguing topic in materials science because of the spectacular changes in relaxation time and mechanical properties that occur precipitously upon reducing temperature [1-3]. Colloidal fluids are excellent model systems for studying glassy dynamics since not only they can reveal very detailed information about kinetic and equilibrium behaviors at experimentally accessible length and timescales on the singleparticle level $[4,5]$, but also the volume fraction (rather than temperature) can be used as a control parameter [5, 6].

Upon increasing the volume fraction, thus approaching the glass transition, colloidal dynamics slows down dramatically. This behavior is not only determined by the direction and the strength of the close-range interactions [7], but also by the shape [8] and topographical surface characteristics of the particles. Although there have been substantial contributions to our understanding of the dynamics of glassy states using colloids with simple shapes (spheres, rods, ellipsoids) and smooth surfaces $[9,10]$, arresting colloidal suspensions in nature and technology often consist of particles with highly irregular shaped and roughness [11-14]. The current lack of studies into more complex particles also contrasts with the diversity and functionality of molecular systems and the recent advances in colloidal science. Many present-day colloids have a more complex architecture [15-17], yet understanding the dynamic interactions of these particles in dense suspensions and the extent of the coupling between the translational and rotational motions remains a standing issue.

Studying this uncharted territory requires experiments that resolve the thermal motions of individual particles. Remarkably, the few systems studied so far displayed opposing trends at the level of mean squared displacements (linear and angular): while colloidal tetramers suspended in a sea of spheres showed that rotations slow down more dramatically than translations [18], the opposite trend was reported for smooth Janus spheres [19]. Colloidal spheres with corrugated surfaces [20], more closely resembling the smooth spheres, showed the rotations to become slightly subdiffusive, while the roughness was found to affect the rotations more strongly than the translations.

The current lack of an encompassing understanding is aggravated by the unclear role of surface inhomogeneities and topography as present on many practical 
colloids. Even minor deviations from smooth isotropic shapes and irregularities on the topography could alter the interplay between the rotational and translational dynamics if the particles get close enough [21]. Even though deviations from smooth surface topography have been shown to generate a broad diversity of macroscopic behaviors such as shear thickening and directed selfassembly $[22,23]$, the interplay between microscopic dynamics and roughness has yet to be resolved.

This chapter provides a unifying insight into colloidal suspensions' dynamics emerging due to a topography alteration to smooth spherical particles. Using our recently developed rotational probes with well-defined asperities [24] and timeresolved 3D confocal microscopy, we show that (unlike their smooth counterparts) rough particles undergo freezing in rotation as well as translation, resulting in two distinct parts glass transitions. Mapping rotational dynamics onto the number of particle-particle contacts supports the underlying physical picture that rotational slowdown can be driven by geometric frustration due to interlocking contacts among the particles.

\subsection{Materials and Methods}

\subsubsection{Materials}

Deionized water (resistivity: $18 \mathrm{M} \Omega . \mathrm{cm}$ ) was obtained from a Millipore Synergy instrument. Core silica $\left(\mathrm{SiO}_{2}\right)$ particles $(\mathrm{r}=1.05 \mu \mathrm{m})$ with amine $\left(\mathrm{NH}_{2}\right)$ surface modification were purchased from Microparticles $\mathrm{GmbH}$. Two types of smaller $\mathrm{SiO}_{2}$ particles (berries) were used: fluorescent spheres (sicastar-greenF, plain, $\mathrm{r}=0.15 \mu \mathrm{m}$, labeled with Fluorescein-IsoThioCyanate (FITC)) were purchased from Micromod Partikeltechnologie $\mathrm{GmbH}$, while plain $\mathrm{SiO}_{2}$ particles were synthesized via the Stöber method [24]. TetraEthoxySilane, $25.0 \mathrm{wt} \%$ Ammonia, analytical grade Ethanol (99.9\%, Emsol), $0.10 \mathrm{~N}$ Nitric Acid solution $\left(\mathrm{HNO}_{3}\right)$, glycerol (99.5\%), fluorescein dye and $\mathrm{LiCl}$ were purchased from Sigma Aldrich. All purchased chemicals were used as received.

\subsubsection{Synthesis and Sample Preparation}

Our experimental system consists of all silica, raspberry-like colloidal particles made via the assembly of many small spheres onto a larger one. The synthesis of the particles is described in our previous work [25]. Briefly, raspberry particles were made by coating positively charged $\left(\mathrm{NH}_{2}\right.$-functionalized) $\mathrm{SiO}_{2}$ spheres 
(cores, $\mathrm{r}_{\mathrm{c}}=1.05 \mu \mathrm{m}$ ) with a dense layer of negatively charged small $\mathrm{SiO}_{2}$ spheres (berries, $r_{b}=0.150 \mu \mathrm{m}$ ) via electrostatic heteroaggregation in an aqueous medium. The berries cover the cores at high density yet still leave space for asperity contacts (Figure 4.1.a). By supplementing the berries with 2-3\% of equally sized fluorescent spheres prior to the reaction with the cores, raspberries with typically 4-6 fluorescent tracers were obtained, thereby creating the optical anisotropy required for measuring particle rotations. These fluorescent raspberries were mixed with non-fluorescent ones to facilitate the identification of individual raspberries (Figure 4.1.b). All colloidal raspberries were coated with a 5-20 nm thin silica layer to ensure mechanical integrity. The surface roughness of the particles was quantified by analyzing Atomic Force Microscopy (AFM) height profiles for several raspberries. A spherical fit to the surface was subtracted from the height profile to isolate the roughness profile [25, 26] (Figure 4.1.c). After centrifugation the particles $\left(\rho_{\mathrm{p}}=1.68 \mathrm{~g} / \mathrm{mL}\right)$ were dispersed in refractive index matching $\left(\mathrm{n}_{\mathrm{d}}=1.44\right)$ mixture of water-glycerol ( $1: 4$ by wt., $\left.\rho_{\mathrm{s}}=1.20 \mathrm{~g} / \mathrm{mL}\right)$ to suppress Van der Waals interactions. $1 \mathrm{mM}$ of fluorescein dye was added to the solvent to visualize and localize all raspberries regardless of fluorescent labels. Additionally, $1 \mathrm{mM} \mathrm{LiCl}$ was added to suppress the electrostatic interactions, giving a Debye length $\mathrm{K}^{-1}$ of $\sim 8 \mathrm{~nm}$. Ten target concentrations ranging from dilute to the glassy limit were prepared. More details of these procedures are given in Supplemental Material Section I.

\subsubsection{Confocal Microscopy Experiments}

Samples were imaged using a confocal scanning laser microscope (CSLM) with a VisiTech 'VT-infinity3' scan unit, a $488 \mathrm{~nm}$ laser and 100x objective (1.49 N.A., oil immersion) connected to a Nikon Eclipse inverted microscope. 3D time series of $\sim 61 \times 60 \times 8-12 \mu \mathrm{m}^{3}$ volumes were captured at fixed time intervals, chosen between 5.0 and $8.1 \mathrm{~s}$. Since the solvent and the fluorescently labeled particles contain dyes with similar excitation wavelengths, we optimized the solvent dye concentration to achieve different intensity levels between the two. This allowed us to visualize all the particles regardless of the presence of fluorescent labels. Here the intensity inverted images are used to localize the centers of all raspberry particles, while the fluorescence of tracer berries was used for tracking the translations and rotations of labeled raspberries. The lowest focal plane was taken at least $10 \mu \mathrm{m}$ above the glass bottom to avoid wall effects. 


\subsubsection{Data Analysis}

3D localization of fluorescent berries and host particles was done using publicly available routines [27-29]. We used the method described in our previous work to extract and separate the tracer raspberries' rotations and translations [25]. By localizing at least four fluorescently labeled berries (per core), we determine a unique center-of-mass location by fitting a sphere encompassing the fluorescent berries' locations. Only the berries separated from each other by at least one berry size were analyzed to ensure correct assignment to a tracer raspberry in a crowded system. Lagtimes for which there were less than 50 mean squared (angular) displacement contributions were not taken into processing. After these filtering steps, we typically obtained $\sim 20-30$ (translational and rotational) trajectories lasting between 100-400s. Translation of each tracer raspberry is then calculated from the time dependence of its center location. The latter location also provides an origin in a 3D Cartesian coordinate system that defines the tracer probe's orientation based on the angular positions of the fluorescent berries [30]. Rotational trajectories are then obtained by extracting the relative change in the angular positions in terms of rigid body transformations [25, 30]. Extensive details on the data analysis are given in our previous work [25] and chapter 3.

a)

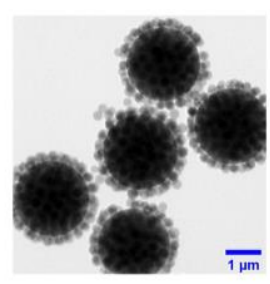

b)

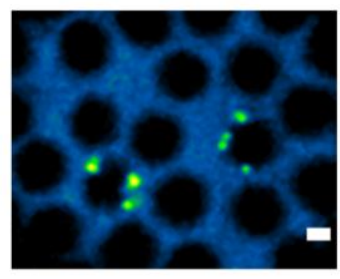

c)

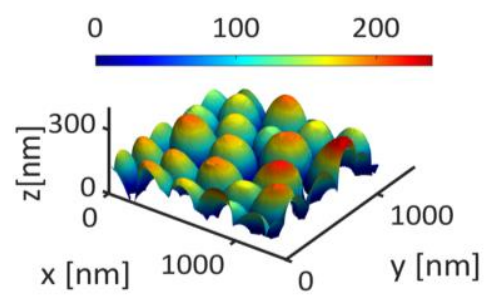

Figure. 4.1. a) TEM image of raspberry particles, b) 2D CSLM image showing the contrast between particles, tracer berries and solvent (false-colored, white scale bar $1 \mu \mathrm{m}$ ), c) Typical height profile of a raspberry (obtained from AFM measurements) after subtracting the curvature of the core particle. 


\subsection{Results and Discussion}

\subsubsection{Effect of Roughness on Translational and Rotational Dynamics}

Centrifuging the suspensions (at a gravitational Peclet number of 1200) into CSLM cuvettes allowed us to directly assess the structure of the suspension, particle number density and translational Brownian motion at the highest achievable concentration in bulk (i.e., more than $10 \mu \mathrm{m}$ from the bottom wall). This suspension displayed a lack of long-range order, while the translational Mean Squared Displacement showed no dependence on lagtime within the investigated time frame (see Figure 4.2.a), thus indicating glassy arrest. Multiplying the number density with the volume per raspberry, we find a volume fraction of $0.54 \pm 0.02$, i.e., significantly below the value of $0.62 \pm 0.02$, which we confirmed for a centrifuged suspension of monodisperse smooth spheres. This shift agrees with literature, where roughness $[22,31]$ and friction $[32,33]$ were found to lower the maximum packing fraction in the glassy state. Note that for raspberry particles, the definition of volume fraction is not entirely trivial. Therefore, when studying the glass transition, the volume excluded to other raspberries (rather than the volume excluded to solvent) should be used. Based on the dense assembly of small spheres in the raspberry shell (see inset Figure 4.2), we estimate the effective raspberry volume to be $9.2 \mu \mathrm{m}^{3}$ (see also Appendix). This corresponds to a smooth sphere with an effective radius $\mathrm{r}_{\mathrm{eff}}=1.30 \mu \mathrm{m}$. The volume fraction dependent Mean Squared Displacements $\left.\left(<\Delta \mathrm{r}^{2}\right\rangle\right)$ and Mean Squared Angular Displacements $\left.\left(<\Delta \theta^{2}\right\rangle\right)$ were measured at lagtimes that mostly ranged from 5 to 100 s, i.e., typically shorter than the characteristic time scales (obtained from Stokes-Einstein, Stokes-Einstein-Debye and $\left\langle\Delta \mathrm{r}^{2}\right\rangle,\left\langle\Delta \theta^{2}\right\rangle$ expressions) for translational (88 $\mathrm{s}$ ) and rotational (119 $\left.\mathrm{s}\right)$ Brownian motion in the dilute limit. 
a)

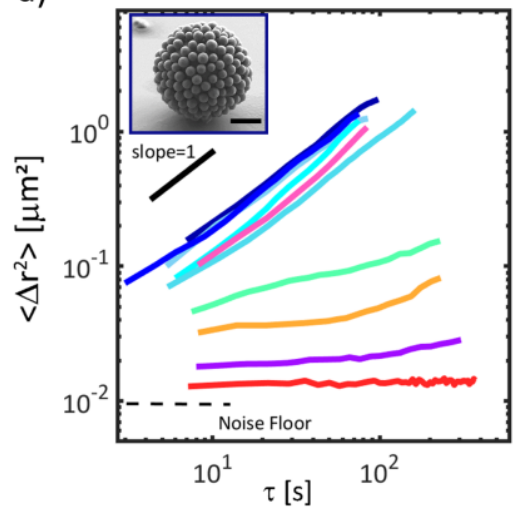

b)

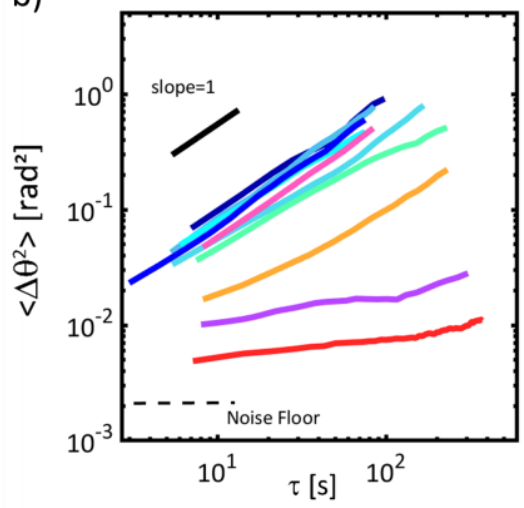

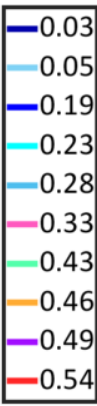

Figure 4.2. (a,b) Volume fraction $(\varphi)$ and lagtime $(\tau)$ dependent $\left\langle\Delta \mathrm{r}^{2}\right\rangle$ and $\left\langle\Delta \theta^{2}\right\rangle$, based on the same image data per $\varphi$. Maximum packing is achieved at $\varphi=0.54$. Whereas the translational diffusion plateaus for $\varphi>0.46$, the diffusive rotational motion is sustained longer and becomes subdiffusive, ultimately plateauing at $\varphi=0.54$. Noise floors are indicated with dashed lines. Inset: SEM image of a raspberry particle, scale bar $=1 \mu \mathrm{m}$

For the translational dynamics (Figure 4.2.a), the typical diffusive signature of colloidal (smooth) hard spheres in the dilute limit is found to extend up to $\varphi$ values as high as 0.33 , while the amplitude of the $\left\langle\Delta \mathrm{r}^{2}\right\rangle$ undergoes a modest reduction. More drastic changes are found between $\varphi=0.33$ and 0.43 , where a subdiffusive regime emerges. On further increasing the concentration, $\left\langle\Delta \mathrm{r}^{2}\right\rangle$ shows plateaus for $\varphi \geq 0.46$, indicating that the particles become dynamically caged by their neighbors at the timescales of our measurement. The progressively lower $\left\langle\Delta \mathrm{r}^{2}\right\rangle$ plateaus at higher $\varphi$ values reflect a further tightening of the cages, to the point that the motion amplitude approaches the noise floor. While this trend is qualitatively similar to that of smooth hard spheres [19], we recall that the maximum achievable volume fraction is now much lower at $\varphi=0.54$.

The rotational mean square displacements (Figure 4.2.b) follow a similar trend with concentration, but the onset volume fractions for subdiffusive behavior and plateau formation are both significantly higher. Notably, at $\varphi=0.46$, where the $\left\langle\Delta \mathrm{r}^{2}\right\rangle$ has already developed an initial plateau, $\left\langle\Delta \theta^{2}\right\rangle$ still shows (almost) diffusive behavior. The transition to a $\left\langle\Delta \theta^{2}>\right.$ plateau is observed at $\varphi=0.49$ and continues up to $\varphi=0.54$, where the $\left\langle\Delta \theta^{2}>\right.$ levels are still significantly above the noise floor. This striking offset in the volume fractions, where the translation and 
the rotation get frozen, reveals -for the first time- two clearly separated glass transitions for rough spherical colloids. The distinct transition regimes in translational and rotational dynamics can further be seen from the volume fraction dependent powerlaw exponents, $\alpha$, of the $\left\langle\Delta \theta^{2}\right\rangle$ and $\left\langle\Delta \mathrm{r}^{2}\right\rangle$ (Figure 4.3.a) and from the ratio between the translational and rotational characteristic times $\left(\tau_{\mathrm{R}}, \tau_{\mathrm{T}}\right)$ (Figure 4.3.b).

In Figure. 3a, the slopes are obtained from linear fits using lag times between 5 to $77 \mathrm{~s}$, where the data are most accurate. Three distinct regimes are observed. In regime I, both translations and rotations are diffusive, suggesting that the particle concentration influences the Brownian motions via the macroscopic viscosity of the suspension. In regime II, the signature of $\left\langle\Delta \mathrm{r}^{2}\right\rangle$ steeply transitions into a subdiffusive one, while $\left\langle\Delta \theta^{2}>\right.$ remains diffusive. This separation of dynamic signatures resembles that of smooth, hard (Janus) spheres in the translationally glassy regime [19]. In regime III, both motions have become strongly subdiffusive. Ultimately both $\left\langle\Delta \mathrm{r}^{2}\right\rangle$ and $\left\langle\Delta \theta^{2}\right\rangle$ reach (near) zero slopes, indicating that only 'rattling' motions are possible. Referring to Figure $4.2 \mathrm{~b}$, we emphasize that the detected rotational motions at the highest concentration are well above the noise floor. We can thus conclude that the rotational rattling involves amplitudes up to $\approx 0.1$ radians.

Figure 4.3.b illustrates another aspect of the dual glass transition with clear separation. Defining $\tau_{\mathrm{R}}$ and $\tau_{\mathrm{T}}$ as the typical times to rotate $1 \pi \mathrm{rad}$, respectively translate $r_{\text {eff, }}, \tau \tau_{\mathrm{R}}$ and $\tau_{\mathrm{T}}$ were obtained from mean squared (angular) displacement functions after replacing them with the best fits and extrapolating where necessary. By plotting the ratio $\tau_{\mathrm{T}} / \tau_{\mathrm{R}}$ we can examine the relative slowdown of translation and rotation on increasing $\varphi$. As similar to the three distinct regimes observed in Figure 4.3.a, translations slow down drastically before the rotations do. 

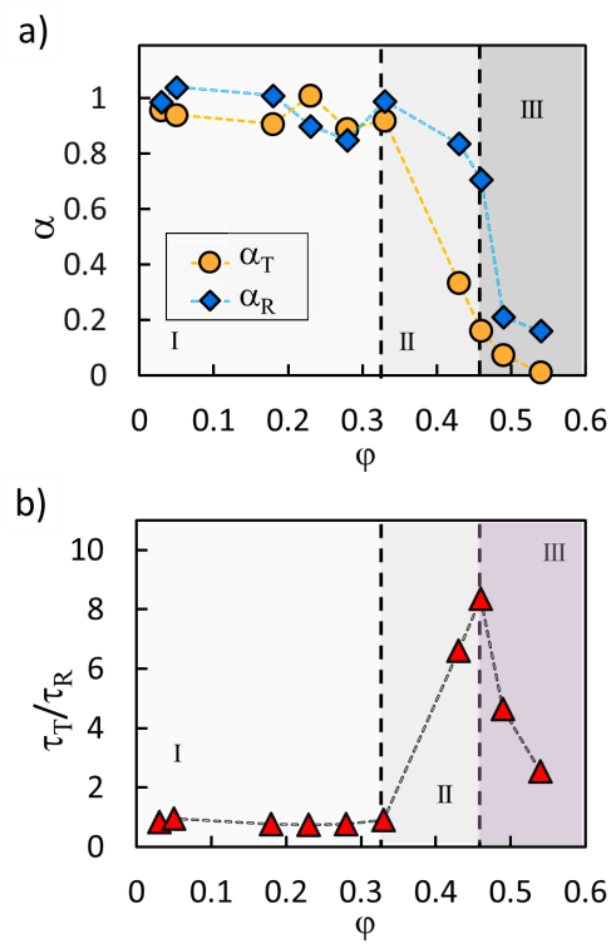

Figure 4.3. Differences in the slowdown of the two types of Brownian motion as $\varphi$ gets higher. a) Log-log slope of the ensemble-averaged $\left\langle\Delta \mathrm{r}^{2}\right\rangle$ and $\left\langle\Delta \theta^{2}\right\rangle$ versus lagtime. Slopes are obtained from linear fits using lag times between 5 to 77 s. b) Ratio of translational to rotational characteristic time.

The question is, how to understand this influence of surface roughness on particle dynamics from a mechanistic point of view. Thanks to the refractive index matching solvent and added salt, the Van der Waals and electrostatic interactions are strongly suppressed. The dynamics of the colloidal raspberries should thus be dominated by excluded volume and hydrodynamic interactions. Similar to smooth, hard spheres [34-36] at low to intermediate volume fractions $(\varphi \leq 0.33)$, where the raspberry surfaces are separated by distances comparable to the particle radius, the hydrodynamic interactions between the overall particles should dominate. Bringing the particles closer together, the translational motions become constrained due to steric caging by surrounding particles. At still closer distances, contributions from the surface morphology start to play a role and eventually become dominant, causing the maximum achievable volume fraction 
to reduce to 0.54 . Also, the differences in rotational dynamics between our raspberries and smooth, hard spheres (SHS) could be explained using only hydrodynamic and steric interactions: at very high densities, the dynamics of SHS would be dominated by lubrication, while the rotational rattling behavior of the raspberries would also require steric interactions.

\subsubsection{Physical Picture of Rotational Slowdown}

Before this work, no rotational glass transition was reported for near-spherical colloids. Recently studied 'smooth' polymer latex (Janus) spheres showed a modest reduction of rotational motion while preserving the diffusive signature, even at very high volume fractions [19]. Polymer spheres with enhanced roughness due to surface crosslinking displayed a transition into a subdiffusive rotational regime [20] without reaching the rotational rattling as in the present work. We, therefore, hypothesize that the distinct rotational glass transition can only be observed if the normal (amplitude) and lateral (spacing) roughness length scales are appropriately sized to allow interlocking, as sketched in Figure 4.4a. When the raspberry particles are close enough, the asperities of one particle penetrating the void spaces of the other particle can generate interlocking configurations which restrict the angular degree of freedom to a narrow range. 
a)

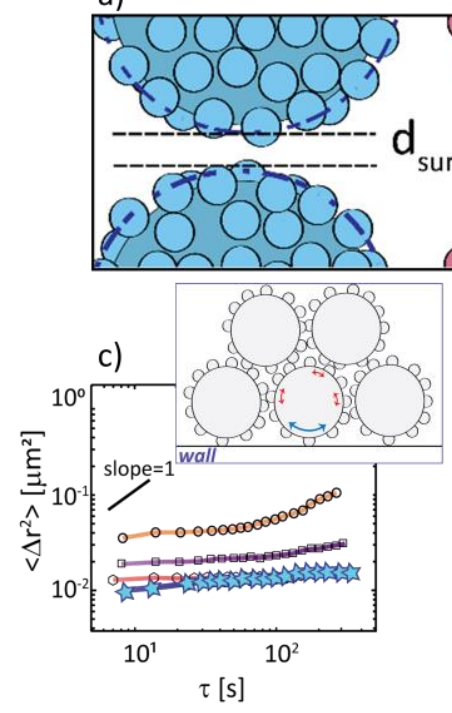

b)

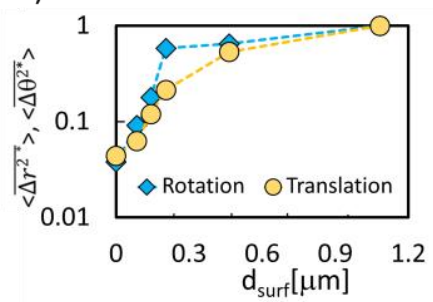

e)

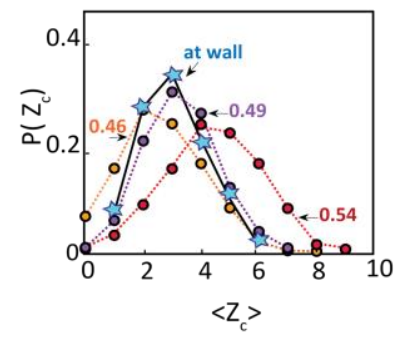

Figure 4.4. a) Artist impression of geometric interaction, with $d_{\text {surf }}$ the intersurface distance. Dashed lines around the raspberries represent the effective smooth radius $r_{\text {eff }} b$ ) Amplitudes of $\left\langle\Delta \mathrm{r}^{2}\right\rangle$ and $\left\langle\Delta \theta^{2}\right\rangle$ at $\tau=15 \mathrm{~s}$, normalized by their values at $\varphi=0.19$, plotted versus $\mathrm{d}_{\text {surf. }}$ Rotation decreases drastically for $\mathrm{d}_{\text {surf }}<0.2 \mu \mathrm{m}$. c-d) $\left\langle\Delta \mathrm{r}^{2}\right\rangle$ and $\left\langle\Delta \theta^{2}\right\rangle$ vs $\tau$ for $\varphi=0.46,0.49,0.54$ and dense layer $\left(\varphi_{2 \mathrm{D}} \cong 0.59\right)$ of particles at the wall (respective colors and symbols: orange circle, purple square, red hexagon, blue star). Inset: Sketch of raspberries in contact with the wall. Red and blue arrows depict rotationally arrested and free contacts, respectively. e) Contact number distribution for the same systems as in panel c-d.

To further reveal the link between the geometric frustration in rotational motion and particles' proximity, we plot the concentration-dependent $\left\langle\Delta \theta^{2}\right\rangle$ and $\left\langle\Delta \mathrm{r}^{2}\right\rangle$ amplitudes against the average intersurface distance $d_{\text {surf }}$ in Figure $4.4 \mathrm{~b}$. A simplistic estimate of $\mathrm{d}_{\text {surf }}$ is obtained by assuming that all the particles are in direct contact at the maximum packing fraction $(\varphi=0.54)$, and lower volume fractions are reached via isotropic expansion on moving away from that point [37].

$$
\mathrm{d}_{\text {surf }}=2 \mathrm{r}_{\text {eff }}\left[\left(\varphi_{\max } / \varphi\right)^{1 / 3}-1\right]
$$


For comparison, we also calculate the average size of the cage $\mathrm{d}_{\text {cage }}$ available to the raspberries for translational rattling. Considering that the apparent $\left\langle\Delta \mathrm{r}^{2}\right\rangle$ plateau is an indication of caging behavior for dense colloidal systems near the glass transition; we estimated $\mathrm{d}_{\text {cage }}$ as $\left\langle\Delta \mathrm{r}^{2}\left(\tau^{*}\right)\right\rangle^{0.5}$. Here, $\tau^{*}$ serves as a representative lagtime and is chosen to be $\sim 15 \mathrm{~s}$ (where the $\left\langle\Delta \mathrm{r}^{2}\right\rangle$ plateaus are significant for $\varphi=0.46-0.54$ ).

Considering that neither distance measure is very precise, the correspondence between the calculated numbers in Table 4.1, where $\mathrm{d}_{\text {cage }}$ should correspond to $2 \mathrm{~d}_{\text {surf }}$, is satisfactory. Both Figure 4.4.b and Table 4.1 indicate that the rotational slowdown becomes prominent for distances $d_{\text {surf }} \leq 0.2 \mu \mathrm{m}$. This value is consistent with the amplitude of the roughness $d_{\text {height }}$, as extracted from AFM measurements showing a fairly dense and homogeneous distribution of berries with a peak-tovalley height difference of $0.16-0.20 \mu \mathrm{m}$ and a typical lateral length scale of $\sim 330$ $\mathrm{nm}$. The agreement between these numbers underlines the proposed physical picture of interlocking as sketched in Figure 4.4.a and further corroborates our geometry-based interpretation.

Table 4.1. Intersurface distance, cage size and coordination number of berries in the rotational glassy regime. $d_{\text {surf }}$ at $\varphi_{\max }=0.54$ is taken as zero as all the particles are assumed to be in direct contact.

\begin{tabular}{c|ccc}
$\varphi$ & $\mathrm{d}_{\text {surf }}[\mu \mathrm{m}]$ & $\mathrm{d}_{\text {cage }}[\mu \mathrm{m}]$ & $\left\langle\mathrm{Z}_{\mathrm{c}}\right\rangle$ \\
\hline 0.43 & 0.21 & - & 2.1 \\
0.46 & 0.14 & 0.19 & 2.7 \\
0.49 & 0.09 & 0.14 & 3.3 \\
0.54 & - & 0.12 & 4.3
\end{tabular}

The emergence of (near) interlocking contacts for $\varphi=0.46$ can be regarded as 'switching on' geometric frustration in the rotational degree of freedom. Interestingly, the same behavior can also be mapped by analyzing the number of contacts of each rough particle with other rough surfaces. The number of interlocking contacts, represented by the static coordination number, $\mathrm{Z}_{\mathrm{c}}$, is shown in Figure 4.4e (as distributions) and included in Table 4.1 (as ensemble averages) for the volume fractions in the rotational glassy regime. Here $\mathrm{Z}_{\mathrm{c}}$ is calculated as the number of surrounding particles within a cutoff distance $\mathrm{d}_{\text {cut_off }}$ (taken to be $2.2 * \mathrm{r}_{\mathrm{eff}}$ to account for polydispersity and localization errors). Increasing the 92 
concentration, $\left\langle\mathrm{Z}_{\mathrm{c}}\right\rangle$ shifts to $\approx 4$ at the maximum packing, where the system can be considered close to jamming. This value agrees well with the literature for athermal jammed packings, where $\left\langle Z_{c}\right\rangle$ was found close to $4\left(Z_{c}=d+1\right.$, with $d$ as the dimensionality) for frictional spherical particles [33, 38, 39]. For comparison, we measured $\left\langle Z_{\mathrm{c}}\right\rangle$ for smooth hard spheres to be 5.9.

These outcomes raise the question, to what extent glass transitions could be controlled via the number of interlocking contacts per particle. Mixing rough spheres with smooth ones was already demonstrated as a possible means of controlling the mechanical properties of sheared glassy suspensions [22]. To examine this idea for quiescent fluids, we measured the dynamics of raspberries in contact with a smooth bottom wall (Figure 4.4c, inset). This monolayer of particles forms a dense packing $\left(\varphi_{\mathrm{AF}}=0.59,2 \mathrm{D}\right.$ area fraction, $\varphi_{\mathrm{AF}}>\varphi_{\max }$ due to layering) while having a dense suspension of rough particles on top. Simplistically, the bottom wall could be regarded as a single smooth particle with a much larger radius. In this picture, the number of particle contacts gets reduced via i) the geometrical confinement (the wall allows only one contact while excluding other neighbors) and ii) the wall's smoothness. The $\left\langle\Delta \mathrm{r}^{2}\right\rangle$ and $\left\langle\Delta \theta^{2}\right\rangle$ of this system are shown in Figure. $4 c-d$, along with the results for bulk $\varphi=0.46$ 0.54. It is evident that in this layer, the particle translations are strongly suppressed, with a $\left\langle\Delta \mathrm{r}^{2}\right\rangle$ similar to that of the bulk system at $\varphi_{\max }=0.54$. No indications for a departure from plateau behavior (corresponding to translational cage-breaking) can be observed up to the longest lag time. In contrast, the $\left\langle\Delta \mathrm{r}^{2}\right\rangle$ for the dense layer of rough particles at the smooth wall still shows subdiffusive behavior, with significant amplitudes and a powerlaw exponent $\alpha_{\mathrm{r}} \approx 0.7$ that most closely resembles that of the bulk data at $\varphi=0.46$. Also, a close resemblance in $\left\langle\mathrm{Z}_{\mathrm{c}}\right\rangle$ for the 2 cases can be seen in Figure 4.4.e.

\subsubsection{Correlations between Translational and Rotational Displacements}

The picture in which the translational Brownian motion influences the rotational diffusion by dynamically changing the contact number and distance between nearby raspberries also implies that individual particles' translational and rotational displacements could be correlated. Here the reasoning is that a particle will have more vigorous Brownian motions when its instant location within the cage offers more space (and vice versa). We express the two types of mobilities via the scalar displacements: $\Delta \theta_{\mathrm{i}}$ and $\Delta \mathrm{r}_{\mathrm{i}}$ : 


$$
\begin{aligned}
& \Delta \theta_{\mathrm{i}}(\tau)=\left|\theta_{\mathrm{i}}(\mathrm{t}+\tau)-\theta_{\mathrm{i}}(\mathrm{t})\right| \\
& \Delta \mathrm{r}_{\mathrm{i}}(\tau)=\left|\mathrm{r}_{\mathrm{i}}(\mathrm{t}+\tau)-\mathrm{r}_{\mathrm{i}}(\mathrm{t})\right|
\end{aligned}
$$

where bold symbols indicate (angular) location vectors, the subscript i labels trajectory segments with duration $\tau$, and the vertical bars indicate absolute value notation. We use scalar quantities since only the amplitudes should be correlated with $d_{\text {surf. }}$ The lagtime $\tau$ should ideally be taken as short as possible, since the coupling between $\Delta \mathrm{r}_{\mathrm{i}}$ and $\Delta \theta_{\mathrm{i}}$ is governed by $\mathrm{d}_{\text {surf. Figure }}$ 4.5.a-b shows typical scatter plots of $\Delta \theta$ vs. $\Delta \mathrm{r}$ for two representative volume fractions for a lag time of $15 \mathrm{~s}$. Using the mean values $(\overline{\Delta r}, \overline{\Delta \theta})$ to define the origin, allows inspecting the plots for any asymmetry over the different quadrants. At $\varphi=0.05$, where colloids are far apart, and both the $\left\langle\Delta \mathrm{r}^{2}\right\rangle$ and $\left\langle\Delta \theta^{2}\right\rangle$ are diffusive, the point-cloud looks symmetric with respect to $(\overline{\Delta r}, \overline{\Delta \theta})$, indicating the absence of correlation between translational and rotational displacements. However, at $\varphi=0.49$, where both translations and rotations are subdiffusive, more events where $\Delta \theta$ and $\Delta \mathrm{r}$ are simultaneously larger or smaller than their mean values indicate a positive correlation between 2 motions. 
a)

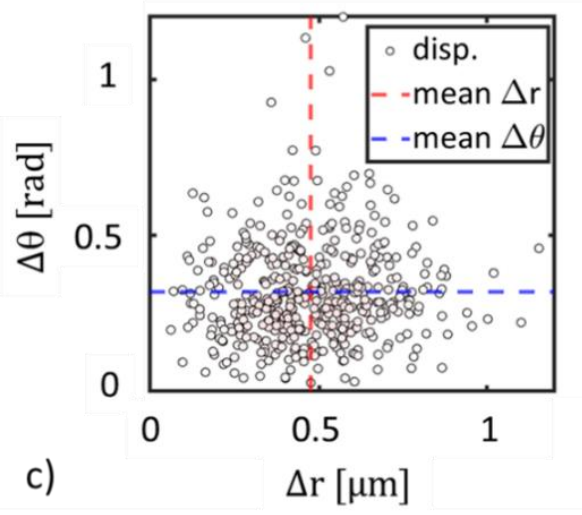

b)

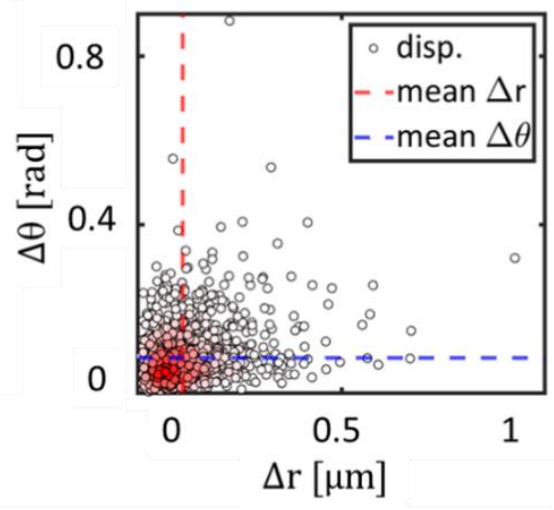

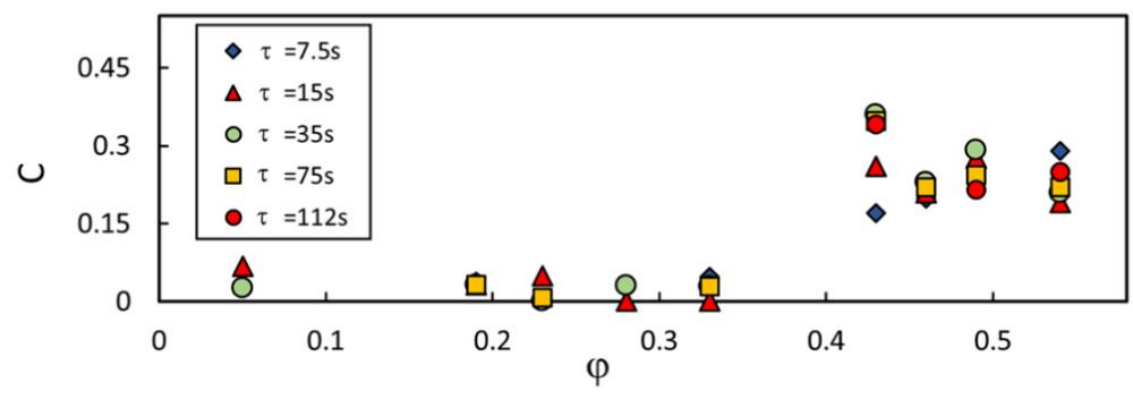

Figure 4.5. Correlations between $\Delta \theta$ vs. $\Delta \mathrm{r}$ for $\tau=15 \mathrm{~s}$. Panels a, b) show scatter plots for volume fractions of 0.05 , and 0.49 and dashed lines indicate the mean values. A color shade of red is given to guide the eye for density populations among the surrounding neighbors, i.e., light to dark: non-crowded to crowded. c) shows the correlation coefficient (see Eq 4.4) obtained for all volume fractions and different lag times. While the motions are non-correlated at low $\varphi$, a significant coupling is observed for $0.43 \leq \varphi \leq 0.54$, where the rotational slowdown becomes prominent.

A more quantitative measure of the correlation is obtained from the lagtimedependent correlation coefficient:

$$
C(\tau)=\frac{\sum_{\mathrm{i}=1}^{\mathrm{n}}\left(\Delta \mathrm{r}_{\mathrm{i}}-\overline{\Delta \mathrm{r}}\right)\left(\Delta \theta_{\mathrm{i}}-\overline{\Delta \theta}\right)}{\sqrt{\sum_{\mathrm{i}=1}^{\mathrm{n}}\left(\Delta \mathrm{r}_{\mathrm{i}}-\overline{\Delta \mathrm{r}}\right)^{2} \sum_{\mathrm{i}=1}^{\mathrm{n}}\left(\Delta \theta_{\mathrm{i}}-\overline{\Delta \theta}\right)^{2}}}
$$

where all $\Delta \theta_{\mathrm{i}}$ and $\Delta \mathrm{r}_{\mathrm{i}}$ depend on $\tau$ as before. Figure 4.5.c shows that regardless of the lagtime, $C$ remains essentially zero up to $\varphi=0.33$. For $\varphi=0.43$ and higher, 
i.e., in the regime of strong rotational slowdown, significant correlations are found. These correlations' magnitudes are similar to those found for tetrahedral colloidal clusters in a supercooled colloidal liquid [18], where (somewhat similar to our case) geometric interactions are prominent. The absence of a clear dependence of $\mathrm{C}$ on lagtime in our data might be related to the finite number of observations (only the tracer berries contribute to the correlations). However, the different behavior of $\mathrm{C}$ in the two concentration regimes is explicit and underlines the picture that $\Delta \theta_{\mathrm{i}}$ and $\Delta \mathrm{r}_{\mathrm{i}}$ are correlated when the particles are in close proximity.

\subsection{Conclusions and Outlook}

Using a novel type of rough colloidal probe spheres allowed us to simultaneously track translations and rotations with unprecedented precision. By studying the dynamics of our model rough colloidal system within a broad concentration range, we show that unlike their smooth counterparts $[1,6]$, rough particles undergo glass transition in rotation as well as translation. On increasing the volume fraction, translational slowdown precedes the rotational one, indicating a distinct separation between two types of glass transition. This hitherto not reported second transition for isotropic-shaped particles reveals rotational rattling of the particles when the particle intersurface distance becomes comparable to the roughness amplitude. It is likely that the particles can then form interlocking contacts leading to geometric frustration. The obtained insights can pave the way for understanding the influence of other types of short-ranged colloidal interactions (electrostatic, Van der Waals, polymer-induced) on the rotational slowdown in glass formation[6, 40]. This should lead to a better understanding of various roughness-related phenomena, including the role of geometric (rolling and sliding) friction in shear-thickening materials $[13,39]$ and the processing of rough particulate systems in industrial applications. In addition, our results can provide a foundation for designing smart materials and superstructures with tunable directional mechanical properties $[15,41]$ that can be obtained by controlling the asperity shape and number density of the surface protrusions. 


\section{Acknowledgments}

We thank Dirk van den Ende and Stefan Luding for helpful discussions. This work was financially supported by NWO-CW (ECHO grant 712.016.004).

\section{Bibliography}

[1] E.R. Weeks, Introduction to the Colloidal Glass Transition, Acs Macro Letters, 6 (2017) 27-34.

[2] P.G. Debenedetti, F.H. Stillinger, Supercooled liquids and the glass transition, Nature, 410 (2001) 259-267.

[3] L. Berthier, G. Biroli, Theoretical perspective on the glass transition and amorphous materials, Reviews of modern physics, 83 (2011) 587.

[4] E.R. Weeks, J.C. Crocker, A.C. Levitt, A. Schofield, D.A. Weitz, Threedimensional direct imaging of structural relaxation near the colloidal glass transition, Science, 287 (2000) 627-631.

[5] P. Pusey, Colloidal glasses, Journal of Physics: Condensed Matter, 20 (2008) 494202.

[6] P.J. Lu, D.A. Weitz, Colloidal particles: crystals, glasses, and gels, Annu. Rev. Condens. Matter Phys., 4 (2013) 217-233.

[7] S. Jiang, J. Yan, J.K. Whitmer, S.M. Anthony, E. Luijten, S. Granick, Orientationally glassy crystals of janus spheres, Physical review letters, 112 (2014) 218301.

[8] K. Zhao, T.G. Mason, Frustrated rotator crystals and glasses of Brownian pentagons, Physical review letters, 103 (2009) 208302.

[9] J. Roller, A. Laganapan, J.-M. Meijer, M. Fuchs, A. Zumbusch, Observation of liquid glass in suspensions of ellipsoidal colloids, Proceedings of the National Academy of Sciences, 118 (2021).

[10] E.R. Weeks, D.A. Weitz, Properties of cage rearrangements observed near the colloidal glass transition, Physical Review Letters, 89 (2002). 
[11] R. Angelini, E. Zaccarelli, F.A. de Melo Marques, M. Sztucki, A. Fluerasu, G. Ruocco, B. Ruzicka, Glass-glass transition during aging of a colloidal clay, Nature communications, 5 (2014) 1-7.

[12] A. Fall, N. Huang, F. Bertrand, G. Ovarlez, D. Bonn, Shear thickening of cornstarch suspensions as a reentrant jamming transition, Physical Review Letters, 100 (2008) 018301.

[13] L.C. Hsiao, S. Pradeep, Experimental synthesis and characterization of rough particles for colloidal and granular rheology, Current Opinion in Colloid \& Interface Science, 43 (2019) 94-112.

[14] E. Blanco, D.J. Hodgson, M. Hermes, R. Besseling, G.L. Hunter, P.M. Chaikin, M.E. Cates, I. Van Damme, W.C. Poon, Conching chocolate is a prototypical transition from frictionally jammed solid to flowable suspension with maximal solid content, Proceedings of the National Academy of Sciences, 116 (2019) 10303-10308.

[15] I. Chakraborty, V. Meester, C. van der Wel, D.J. Kraft, Colloidal joints with designed motion range and tunable joint flexibility, Nanoscale, 9 (2017) 78147821.

[16] S. Sacanna, W.T. Irvine, P.M. Chaikin, D.J. Pine, Lock and key colloids, Nature, 464 (2010) 575-578.

[17] D.J. Kraft, W.S. Vlug, C.M. van Kats, A. van Blaaderen, A. Imhof, W.K. Kegel, Self-assembly of colloids with liquid protrusions, Journal of the American Chemical Society, 131 (2009) 1182-1186.

[18] K.V. Edmond, M.T. Elsesser, G.L. Hunter, D.J. Pine, E.R. Weeks, Decoupling of rotational and translational diffusion in supercooled colloidal fluids, Proceedings of the National Academy of Sciences, 109 (2012) 1789117896.

[19] M. Kim, S.M. Anthony, S.C. Bae, S. Granick, Colloidal rotation near the colloidal glass transition, The Journal of chemical physics, 135 (2011) 054905.

[20] L.C. Hsiao, I. Saha-Dalal, R.G. Larson, M.J. Solomon, Translational and rotational dynamics in dense suspensions of smooth and rough colloids, Soft Matter, 13 (2017) 9229-9236. 
[21] R. Fuchs, T. Weinhart, J. Meyer, H. Zhuang, T. Staedler, X. Jiang, S. Luding, Rolling, sliding and torsion of micron-sized silica particles: experimental, numerical and theoretical analysis, Granular matter, 16 (2014) 281-297.

[22] C.P. Hsu, S.N. Ramakrishna, M. Zanini, N.D. Spencer, L. Isa, Roughnessdependent tribology effects on discontinuous shear thickening, Proceedings of the National Academy of Sciences of the United States of America, 115 (2018) 5117-5122.

[23] M. Kamp, M. Hermes, C.M. Van Kats, D.J. Kraft, W.K. Kegel, M. Dijkstra, A. Van Blaaderen, Selective depletion interactions in mixtures of rough and smooth silica spheres, Langmuir, 32 (2016) 1233-1240.

[24] W. Stöber, A. Fink, E. Bohn, Controlled growth of monodisperse silica spheres in the micron size range, Journal of colloid and interface science, 26 (1968) 62-69.

[25] B. Ilhan, J.J. Schoppink, F. Mugele, M.H.G. Duits, Spherical probes for simultaneous measurement of rotational and translational diffusion in 3 dimensions, Journal of Colloid and Interface Science, 576 (2020) 322-329.

[26] B. Ilhan, C. Annink, D.V. Nguyen, F. Mugele, I. Siretanu, M.H.G. Duits, A method for reversible control over nano-roughness of colloidal particles, Colloids and Surfaces a-Physicochemical and Engineering Aspects, 560 (2019) 50-58.

[27] J.C. Crocker, D.G. Grier, Methods of digital video microscopy for colloidal studies, Journal of colloid and interface science, 179 (1996) 298-310.

[28] K.E. Jensen, N. Nakamura, Note: An iterative algorithm to improve colloidal particle locating, Review of Scientific Instruments, 87 (2016) 066103.

[29] Y. Gao, M.L. Kilfoi, Accurate detection and complete tracking of large populations of features in three dimensions, Optics express, 17 (2009) 4685-4704.

[30] G.L. Hunter, K.V. Edmond, M.T. Elsesser, E.R. Weeks, Tracking rotational diffusion of colloidal clusters, Optics express, 19 (2011) 17189-17202.

[31] S. Pradeep, L.C. Hsiao, Contact criterion for suspensions of smooth and rough colloids, Soft Matter, 16 (2020) 4980-4989. 
[32] H. Shi, S. Roy, T. Weinhart, V. Magnanimo, S. Luding, Steady state rheology of homogeneous and inhomogeneous cohesive granular materials, Granular matter, 22 (2020) 14.

[33] L.E. Silbert, Jamming of frictional spheres and random loose packing, Soft Matter, 6 (2010) 2918-2924.

[34] S. Schütter, J. Roller, A. Kick, J.-M. Meijer, A. Zumbusch, Real-space imaging of translational and rotational dynamics of hard spheres from the fluid to the crystal, Soft matter, 13 (2017) 8240-8249.

[35] V. Degiorgio, R. Piazza, R.B. Jones, Rotational diffusion in concentrated colloidal dispersions of hard spheres, Physical Review E, 52 (1995) 2707.

[36] A.J. Banchio, G. Nägele, Short-time transport properties in dense suspensions: from neutral to charge-stabilized colloidal spheres, The Journal of chemical physics, 128 (2008) 104903.

[37] K.H. deHaas, C. Blom, D. vandenEnde, M.H.G. Duits, B. Haveman, J. Mellema, Rheological behavior of a dispersion of small lipid bilayer vesicles, Langmuir, 13 (1997) 6658-6668.

[38] J.F. Morris, Shear Thickening of Concentrated Suspensions: Recent Developments and Relation to Other Phenomena, Annual Review of Fluid Mechanics, Vol 52, 52 (2020) 121-144.

[39] A. Singh, C. Ness, R. Seto, J.J. de Pablo, H.M. Jaeger, Shear Thickening and Jamming of Dense Suspensions: The "Roll" of Friction, Physical Review Letters, 124 (2020).

[40] G.L. Hunter, E.R. Weeks, The physics of the colloidal glass transition, Reports on Progress in Physics, 75 (2012).

[41] D.J. Kraft, R. Ni, F. Smallenburg, M. Hermes, K. Yoon, D.A. Weitz, A. van Blaaderen, J. Groenewold, M. Dijkstra, W.K. Kegel, Surface roughness directed self-assembly of patchy particles into colloidal micelles, Proceedings of the National Academy of Sciences, 109 (2012) 10787-10792. 


\section{Appendix}

\section{Experimental Details}

Suspension preparation: Colloidal suspensions were prepared at 10 volume fractions pranging from 0.03 to 0.54 . In each system, $3-4 \%$ of the raspberries contained fluorescent labels. A stock suspension was concentrated via soft centrifugation $(\sim 200 \mathrm{~g})$ followed by redispersion into the calculated amount of solvent, assuming no excess mixing volume. Here a particle mass density $\rho_{\mathrm{p}}=1.68$ $\mathrm{g} / \mathrm{mL}$ was used whereas; it was $\rho_{\mathrm{s}}=1.2 \mathrm{~g} / \mathrm{mL}$ for the dispersing solvent. The latter was a water-glycerol mixture at a $\sim 1: 4$ mass ratio, with a refractive index $n_{s}=1.44$, i.e., equal to that of the $\mathrm{SiO}_{2}$. Since our particles have a negative buoyancy in the used solvent, all prepared suspensions developed some concentration gradients over time, thereby increasing the particle volume fraction near the bottom of the sample container in which the Confocal Scanning Laser Microscopy (CSLM) observations were done. This process's speed strongly depends on particle concentration: on decreasing $\varphi$, the local $\mathrm{d} \varphi / \mathrm{dt}$ got progressively larger.

Nevertheless, as we show, the dependence of the $\left\langle\Delta \mathrm{r}^{2}\right\rangle$ and $\left\langle\Delta \theta^{2}\right\rangle$ on $\varphi$ is relatively weak at low volume fractions. Therefore, suspensions at low to intermediate concentrations $(\varphi \leq 0.33)$ were prepared by simply redispersing the sediment into the amount of solvent required to reach the target concentration. Measurements of the Brownian motions with CSLM were started shortly after homogenization, and the value of $\varphi$ was checked from concurrently recorded image stacks. Volume fractions in the range of $0.43<\varphi<0.49$, where gravitational settling was relatively slow, were prepared using the negative buoyancy. For these samples, long CSLM recordings were made to capture both the particle dynamics and the local volume fraction evolution. The experimental time span corresponding to a given $\varphi$ was then selected posterior, based on the CSLM data. Finally, the suspension at $\varphi=0.54$ was obtained via second centrifugation, this time without redispersion. 


\section{Estimation Of Volume Fractions}

\section{Calculation of Volume Fraction from Number Density}

Reported volume fractions $\varphi$, were calculated by performing a 3D localization of all raspberries and multiplying the measured number density with $(4 \Pi / 3) \mathrm{r}_{\mathrm{eff}}{ }^{3}$. This method has often been used for smooth spheres. While the number density can be measured with good precision (in our case, particle identification is very reliable, and we typically have $>1000$ particles in the imaged volume), a precise (independent) measurement of the particle radius is more critical for the absolute accuracy in $\varphi$ [1]. To account for our particles' surface roughness, we fitted an equivalent sphere through the berries' centers and supplemented it with the hemispherical volumes of the 300 berries. This resulted in an effective radius $r_{\text {eff }}$ $=1.3 \mu \mathrm{m}$, which is slightly smaller than the outermost radius $\left(\mathrm{r}_{\text {core }}+2 \mathrm{r}_{\text {berry }}\right)$ and corresponds well with earlier measurements of the hydrodynamic radius [2]. Using $\mathrm{r}_{\mathrm{eff}}=1.3 \mu \mathrm{m}$, the maximum packing fraction achieved in the present study is 0.54 . Using the outermost radius would have resulted in a value of 0.59 .

\section{Measurement of Area Fraction after Intensity Thresholding}

We used horizontal (X, Y) CSLM images to quantify the area fraction (AF) that was not occupied by fluorescent solvent as a function of the $(\mathrm{Z})$ height in the sediment. Local $\varphi$ values were calculated by averaging $(X, Y)$ images over a certain Z-range. Otsu's binarization method was used with an intensity threshold that was automatically adjusted per slice [3] (See Figure A1). Bright pixels corresponding to fluorescent berries were not separated during intensity thresholding since they typically occupied only $\sim 0.2 \%$ of the area per $\mathrm{Z}$ slice. Representative concentration vs. height profiles as measured during sedimentation are shown in Figure A2; the variation of $\varphi$ with time and Z-location was found to be sufficiently weak to justify averaging them throughout an experiment. 

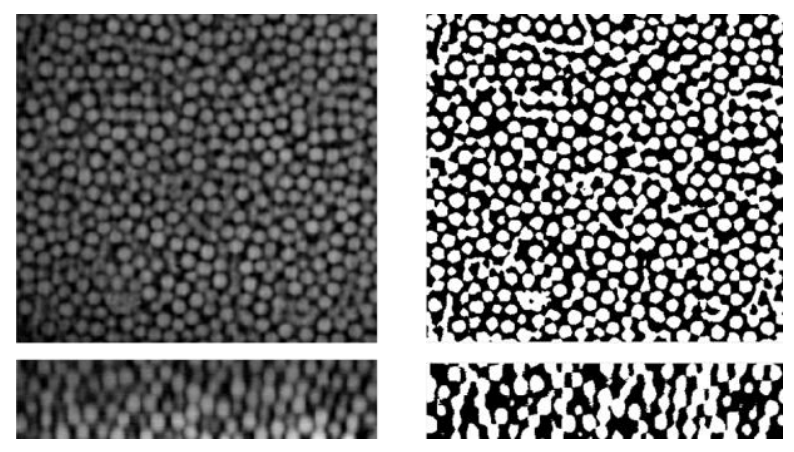

Figure A1. Visualization of the non-fluorescent silica component of our system, at a volume fraction of $\varphi \approx 0.49$. Left: raw data after intensity inversion. Right: after intensity thresholding. Top images: XY, Bottom images: YZ. These images were taken near the maximum depth ( $\mathrm{Z}=17 \mu \mathrm{m}$ from the bottom).

\section{Comparison of Volume Fractions calculated from Area Fraction and Number Density}

The volume fractions obtained from both methods are compared for different suspensions that were all centrifuged into the glassy state. Besides a comparison of the analysis methods also the variability between different samples is demonstrated. 
Table A1. Volume fractions in maximum packing states (obtained via long-time sedimentation and fast centrifugation for E4) were calculated via different methods.

\begin{tabular}{l|l|l} 
Experiment no & AF after thresholding, with $\sigma[\%]$ & $\varphi[\%]$ from number density \\
\hline E1 & $52.0 \pm 1.9$ & 53.0 \\
E2 & $52.6 \pm 1.2$ & 52.2 \\
E3 & $52.9 \pm 0.9$ & 53.3 \\
E4 & $54.7 \pm 1.0$ & 53.5 \\
E5 & $51.9 \pm 0.9$ & 53.2
\end{tabular}

\section{Depth dependence of Area Fraction and its Evolution}

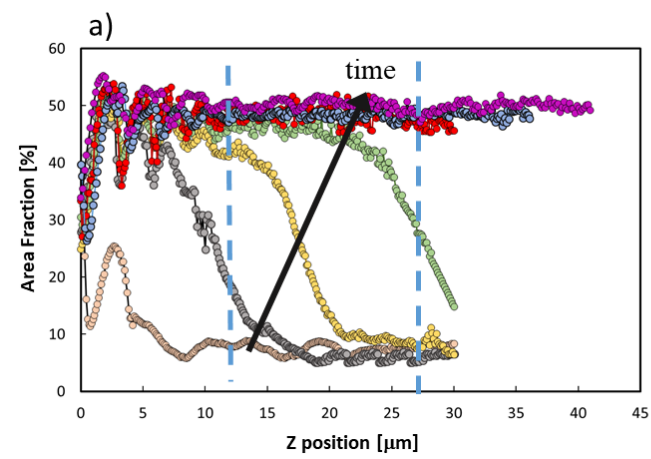

b)

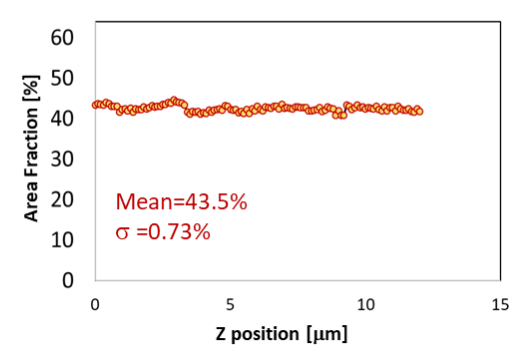

Figure A2. a)Compaction of raspberry suspension over time. Eventually, average area fractions up to $\sim 50 \%$ are obtained. A bulk volume fraction of $8 \%$ allows obtaining intermediate stages where the volume fraction is approximately constant between $\mathrm{Z}=11$ $\mu \mathrm{m}$ and $\mathrm{Z}=19-23 \mu \mathrm{m}$ for the duration of a tracking experiment. Time intervals: $40 \mathrm{~min}$. b): Representative density profile used for the tracking experiment $(\varphi=0.43)$. The minor variations with $\mathrm{Z}$ are attributed to the slice-by-slice binarization. 


\section{Measurement of Maximum Packing Fraction via Binarization and Spatial Variation of Area Fraction in the Steady State}

a)

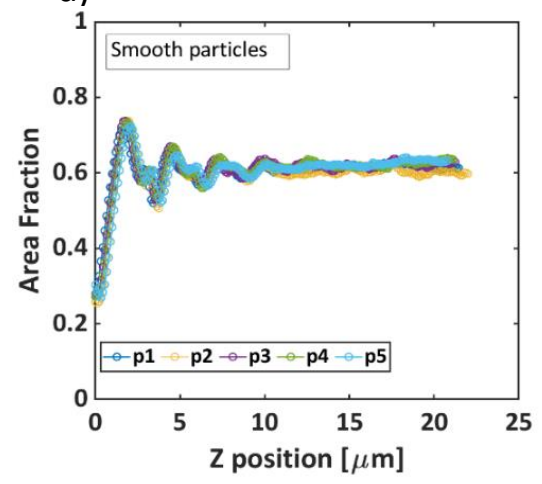

b)

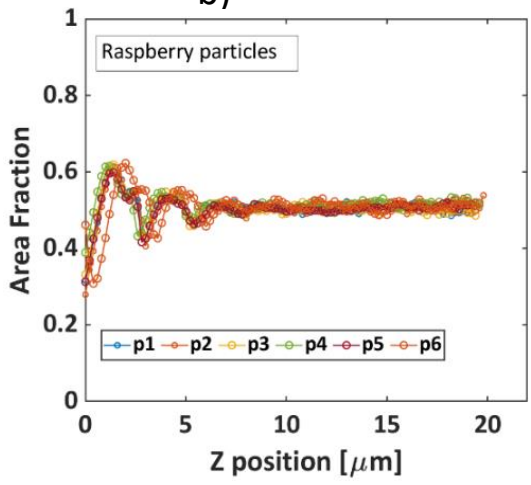

Figure A3. Measurement of the Z-dependent volume fraction for different XY locations. a): smooth spheres $(d=3 \mu \mathrm{m}),(\mathrm{b})$ : raspberries. Measurements are taken right after highspeed centrifugation of both systems to characterize the maximum packing fraction. Different colors represent different locations in the sample. 
Literature Comparison: Maximum Packing Fractions for Rough and Smooth Colloids

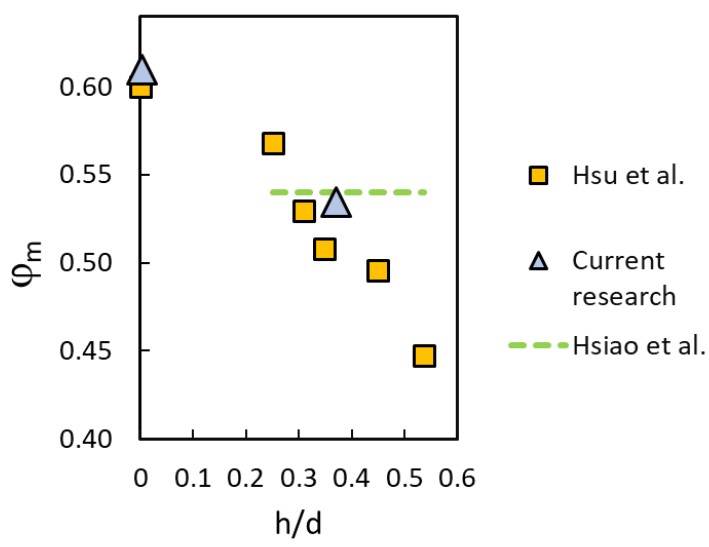

Figure A4. The maximum packing fraction of low-polydispersity colloids (Figure is adapted from Hsu et al.), plotted against roughness measure $\mathrm{h} / \mathrm{d}$ as introduced in Hsu et al. [4]. Here $h$ and $d$ are the typical height and lateral separation of the surface asperities. For our system, h and d are defined similarly. For the data (dashed lines) from Hsiao et al. [5] h/d parameter is not calculated due to differences in roughness geometry

\section{Measurement of Mean Squared Displacements using Solvent Background Fluorescence}

Dissolving fluorescein in the water-glycerol (1:4 by wt) medium allowed to track the motions of all raspberries (a large majority was not fluorescent) in 3 dimensions. Inversion of the grayscale intensities then renders the raspberries as bright objects that can be identified as objects and subsequently localized. Photobleaching of the fluorescein under our imaging conditions $(35-40 \mathrm{~mW})$ provided some limitations to the duration of the exposure. Also, the FITC dye in the fluorescent berries photobleached, but more slowly. 
a)
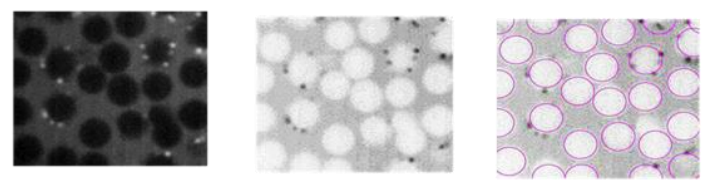

b)
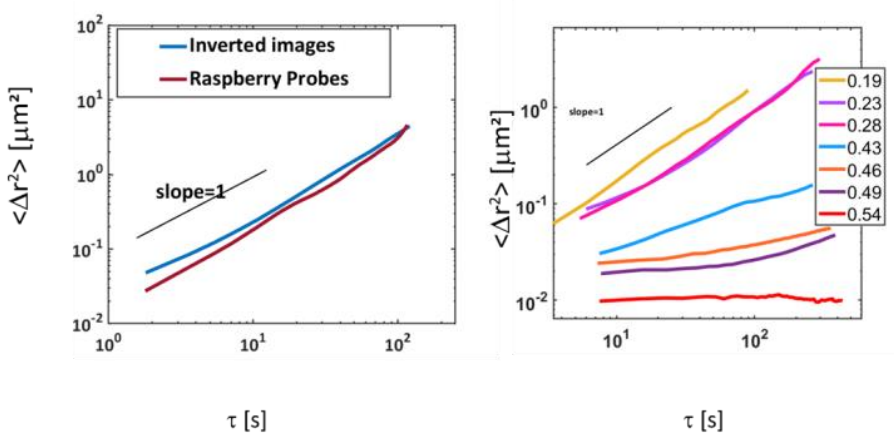

Figure A5. a) Image analysis steps: inversion of the intensity map and localization of bright objects (here raspberries). The center locations of these spheres are used for building the translational particle trajectories. b) Left panel: comparison with $\left\langle\Delta \mathrm{r}^{2}\right\rangle$ measured from the raspberry probes. Right panel: $\left\langle\Delta \mathrm{r}^{2}\right\rangle$ as a function of volume fraction for several $\varphi$.

\section{Details of Fluorescent Tracer Identification and Noise Level Estimation}

In concentrated systems, the berry particles' assignment to their corresponding raspberries can be more challenging since the raspberries will be closer to each other. This issue was mitigated by using a small fraction (2-3\%) of tracer raspberries. To ensure correct assignment to a raspberry, only fluorescent berries that were at least one berry size apart from others were analyzed. After this filtering step, we typically obtained $\sim 20-30$ (translational and rotational) trajectories lasting between 100-400s. It should be noted here that our experiment duration is limited by sedimentation (higher rates at lower volume fractions) and photobleaching of the fluorescent berry particles. The accuracy of the translational tracking was examined via a comparison with the tracking of nonfluorescent host particles (Figure.A5). Noise floors of MS(A)Ds were measured by drying a dispersion of raspberries on a glass coverslip and immersing the dried material in a refractive index matching solvent prior to particle tracking. 


\section{Bibliography}

[1] W.C. Poon, E.R. Weeks, C.P. Royall, On measuring colloidal volume fractions, Soft Matter, 8 (2012) 21-30.

[2] B. Ilhan, J.J. Schoppink, F. Mugele, M.H.G. Duits, Spherical probes for simultaneous measurement of rotational and translational diffusion in 3 dimensions, Journal of Colloid and Interface Science, 576 (2020) 322-329.

[3] D. Le-Anh, A. Rao, S.C. Ayirala, M.B. Alotaibi, M.H.G. Duits, H. Gardeniers, A.A. Yousef, F. Mugele, Optical measurements of oil release from calcite packed beds in microfluidic channels, Microfluidics and Nanofluidics, 24 (2020).

[4] C.P. Hsu, S.N. Ramakrishna, M. Zanini, N.D. Spencer, L. Isa, Roughnessdependent tribology effects on discontinuous shear thickening, Proceedings of the National Academy of Sciences of the United States of America, 115 (2018) 5117-5122.

[5] S. Pradeep, L.C. Hsiao, Contact criterion for suspensions of smooth and rough colloids, Soft Matter, 16 (2020) 4980-4989. 


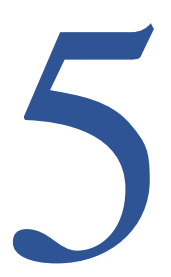

\section{Probing the Effect of Host Size on the Translational and Rotational Dynamics of Rough Tracers}

In concentrated suspensions, the translational and rotational dynamics of colloids are strongly influenced by the topographical characteristics of the particles. When particles are in close proximity, surface roughness and emerging geometric hindrance can alter the interplay between the translational and rotational Brownian motion. Using novel rough probes with well-defined asperities (i.e., colloidal silica raspberries with fluorescent tracers in the shell), we observe the rotational and translational diffusion of rough particles in generic glass forming, host colloidal systems with smooth surfaces. Using two different asperity/host size ratios (1 and 4), we investigate the rotational and translational dynamics as a function of host particle volume fraction. Our results show that the rotational slowdown is due to geometric frustration of the surface asperities with the surrounding system. The effect is the largest when the asperity/host ratio is 1 . The rotational slowdown is concurrent with a change in the Mean Squared Angular Displacement signature from diffusive to rattling behavior. The translational slowdown is less sensitive to the size of the host particles. We benchmark our results with a case where novel spherical probes with a smoothened surface (normal roughness amplitude $<1 \%$ radius) are dispersed in the same size host system. These particles do not exhibit rotational arrest even at the highest concentrations, and the rotational Brownian motion shows a transition from diffusive to mildly subdiffusive. 


\subsection{Introduction}

In colloidal suspensions, upon approaching the glass transition, the viscosity grows by several orders of magnitude, and the Brownian motions of the particles slow down dramatically. This slowing down emerges due to the crowding of the particles. While confined in cages formed by their neighbors, a particle's motion becomes constrained, and escape from the cage requires a collective rearrangement of the surroundings [1-3]. This confinement effect has different implications for translational and rotational degrees of freedom [4, 5]. For example, smooth spherical particles undergo freezing in translations due to their confining cages, but their rotational motion ensues since no cage rearrangement is needed to enable rotation [6]. In contrast, for shape anisotropic particles such as ellipsoids with large aspect ratios, steric cages obstruct not only the translational motion but also the rotational one [7-9]. This means that in dense colloidal suspensions, the dynamics is strongly dependent on the shape of the particles. Due to close-range particle-particle interactions, deviations from smooth topography can alter the confinement length scale for the translational and rotational motion.

The close proximity of the particles in dense suspensions naturally raises the question, how the geometric surface characteristics influence the rotational and translational motions. In the previous chapter, using our model rough colloidal system (i.e., raspberries), we showed that surface roughness causes a geometric frustration in the rotational degrees of freedom due to interlocking configurations among the asperities. This interlocking effect is prominent at high enough concentrations that the interparticle distances are comparable to asperity length scales.

In this chapter, we translate and extend our understanding from Chapter 4 into other glass forming systems. Using our model raspberry particles as a tool for probing the effective length scales for the geometric hindrance, we observe the rotational and translational diffusion of rough particles in generic glass forming host colloidal systems with different sizes. Thus varying the asperity/host size ratio, we quantify the rotational and translational dynamics as a function of host particle volume fraction. We benchmark our results with a system of spherical probes with a smoothened surface in a generic glass-forming host system. 


\subsection{Materials and Methods}

\subsubsection{Materials}

Deionized water (resistivity: $18 \mathrm{M} \Omega . \mathrm{cm}$ ) was obtained from a Millipore Synergy instrument. Core silica $\left(\mathrm{SiO}_{2}\right)$ particles $\left(\mathrm{r}_{\text {core }}=1.05 \mu \mathrm{m}\right)$ with amine $\left(-\mathrm{NH}_{2}\right)$ surface modification were purchased from Microparticles $\mathrm{GmbH}$. Five types of smaller $\mathrm{SiO}_{2}$ particles (berries) were used during the synthesis of the raspberry probes. Fluorescent spheres: sicastar-greenF, plain, $\mathrm{r}_{\text {berries }}=0.15 \mu \mathrm{m}$, labeled with Fluorescein-IsoThioCyanate (FITC); sicastar-redF, plain, $\mathrm{r}_{\text {berries }}=0.15 \mu \mathrm{m}$, labeled with Rhodamine B isothiocyanate (RITC) were purchased from Micromod Partikel-technologie $\mathrm{GmbH}$, while yellow/green fluorescent polystyrene latex particles ( $\mathrm{r}_{\mathrm{PS}}=12.5 \mathrm{~nm}$, sulfate modified) was purchased from Molecular Probes. Ludox TMA colloidal $\mathrm{SiO}_{2}\left(\mathrm{r}_{\text {Ludox }}=11 \mathrm{~nm}\right.$ ) was purchased from Sigma Aldrich. Plain $\mathrm{SiO}_{2}$ particles (both host glass-forming systems and berries) were synthesized via the Stöber method [10]. TetraEthoxySilane, 25.0 wt\% Ammonia, analytical grade Ethanol (99.9\%, Emsol), $0.10 \mathrm{~N}$ Nitric Acid solution $\left(\mathrm{HNO}_{3}\right)$, glycerol (99.5\%), Dimethyl sulfoxide (DMSO, anhydrous >99.9\%) and $\mathrm{LiCl}$ were purchased from Sigma Aldrich. All purchased chemicals were used as received.

\subsubsection{Synthesis of Rough and Smoothened Probes}

Our experimental system consists of rough (raspberry) and smoothened probes.

\section{Rough Probes}

Rough probes are all silica, raspberry-like colloidal particles made via the assembly of many small spheres onto a large spherical core particle. Details of the synthesis procedure and characterization are given in Chapter 2, and our previous work [2]. We used two types of raspberry probes. Both were synthesized with the same protocol and have the same topographical characteristics; their only distinction is the fluorescent labels of the marker berries (FITC or RITC).

Smoothened Probes

The synthesis of smoothened probes is similar to that of raspberry-like particles. Smoothened probes were made via the assembly of many tiny spheres (smaller than rough probes' berries) onto a larger core $\mathrm{SiO}_{2}$ particle $\left(\mathrm{r}_{\text {core }}=1.05 \mu \mathrm{m}\right)$. The 
surface of the positively functionalized (-NH2) $\mathrm{SiO}_{2}$ cores was covered by a mixture of negatively charged Ludox TMA colloidal $\mathrm{SiO}_{2}\left(\mathrm{r}_{\mathrm{Ludox}}=11 \mathrm{~nm}\right)$ and fluorescent PS particles $\left(\mathrm{r}_{\mathrm{PS}}=12.5 \mathrm{~nm}\right)$ via electrostatic hetero-aggregation [11] (See Figure 5.1.a). The reaction was achieved by adding cores to a suspension of a mixture of berry particles ( $1 \% \mathrm{wt}$.) under mild stirring and giving a reaction time of $1 \mathrm{~h}$ in an aqueous solution at $\mathrm{pH}$ 4.5. The amount of berries in the mixture was chosen to be at least ten times excess than required for complete coverage to ensure colloidal stability throughout the self-assembly process. Similar to raspberry probes, the majority of the berries covering the core particle were nonfluorescent $\mathrm{SiO}_{2}$ spheres. To introduce optical anisotropy, only a small fraction of the berries were fluorescent PS particles (see Figure 5.2.b). The proportion of fluorescent berries in the reaction mixture was chosen to be $\sim 3.0 \%$ which typically leads to 4-6 fluorescent berries per particle. After the reaction, excess (unattached) berries were removed by three cycles of gravity settling and redispersion in an aqueous solution at $\mathrm{pH} 4.5$. A $\sim 300 \mathrm{~nm}$ silica layer was overgrown on the probes via seeded growth [12]. This layer ensures the supracolloidal probes' mechanical integrity and further smoothens the asperities on the surface. The final size of the probes is estimated to be between $\mathrm{d}=2.4-2.6 \mu \mathrm{m}$. The surface topography of the particles was quantified by analyzing Atomic Force Microscopy (AFM) height profiles for several probes (See Figure 5.1.c). A spherical fit to the surface was subtracted from the height profile to isolate the roughness profile $[11,13]$.
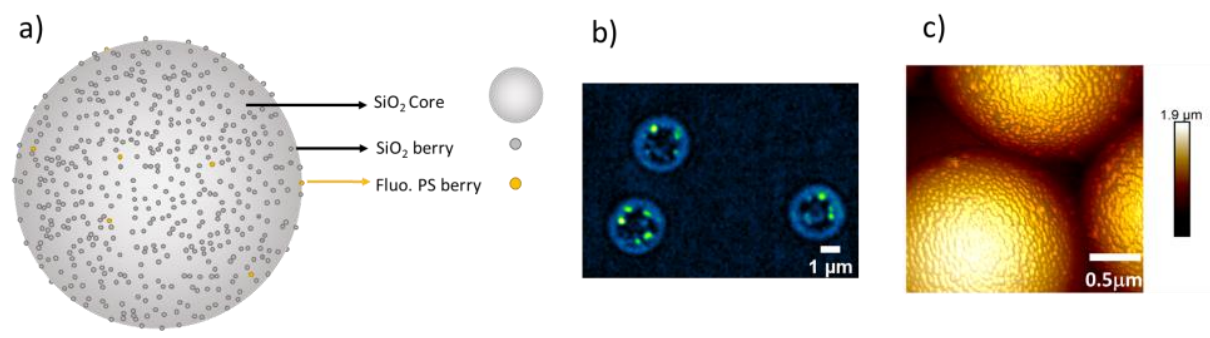

Figure 5.1. a) Schematic drawing of the smoothened probes, b) Superimposed brightfield and fluorescent images of the probes (false-colored), c) 3D rendering of the AFM height profile of several particles.

It should be noted here that the topography still contains asperities even though a relatively thick (compared to berry size) layer was overgrown on the smoothened probes. The probes have a root-mean-square roughness amplitude of around $\sim 8.5$ $\mathrm{nm}$. Indeed this qualifies our probes as surface rough particles especially 112 
compared to pristine colloidal particles, which are usually smoother with an rms roughness smaller than $1 \mathrm{~nm}$ [13].

Here we would like to make a distinction by comparing our probes with the raspberry-like particles. As demonstrated in Chapter 4, in dense suspensions, where particles are in close proximity, the geometric frustration on the particle's degree of freedom is dependent on the length scale of asperities' peak to valley distances ( $R_{\text {max_probe }}$ ). The smoothened probes have $R_{\max }$ around $10-15 \mathrm{~nm}$. Compared to raspberry particles with a $R_{\max }$ amplitude around $200 \mathrm{~nm}$, our smoothened probes are $\sim 95 \%$ smoother, making them highly unlikely to have interlocking configurations. A roughness comparison between the raspberries and the smoothened probes can be found in Figure 5.2.c, where peak-to-valley distances are considerably smaller for the smoothened probes. For ease of understanding and simplicity, hereafter, they will be referred as smooth probes.

a)

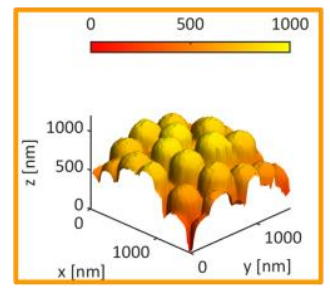

b)

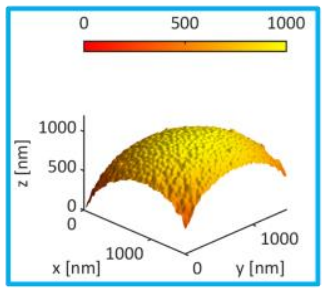

c)

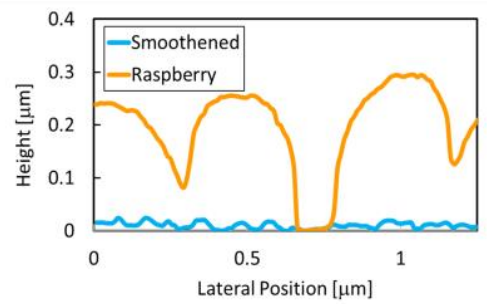

Figure 5.2. Characterization of particle surface roughness using AFM. a) Height profile of a raspberry-like rough probe, b) Height profile of a smoothened probe, c) 2D relative residual asperity height profiles of the two particles after subtracting the curvature of the core(i.e., remaining surface characteristics do not contain sphericity and both raspberry and smoothened systems are in scale with each other.)

\subsubsection{Sample Preparation}

Three different probe-host mixtures in water-DMSO were used. For the rough probes, the ratio $\lambda=l_{\text {asperity }} / \mathrm{d}_{\text {host }}$ between asperity amplitude $\left(l_{\text {asperity }}=R_{\text {max_probe }}\right)$ and diameter of the host particle was taken to be either 1 or 4 . A benchmark case was also studied with smooth probes dispersed in smaller (identical to $\lambda=1$ case) colloidal smooth host spheres. 
Raspberry probes in asperity sized hosts, $\lambda=1$

In each sample, less than $0.5 \%$ of the volume consisted of raspberry probes. The host system consisted of smooth and bidisperse $\mathrm{SiO}_{2}$ particles $\left(\mathrm{r}_{\text {host }}=0.10\right.$ and 0.12 $\mu \mathrm{m}, 1: 1$ by volume, $\rho_{\text {host }}=1.75 \mathrm{~g} / \mathrm{ml}$ ), where the size-mixing was done to avoid crystallization. To minimize Van der Waals attractions, the silica colloids were dispersed in refractive index matching $\left(\mathrm{n}_{\mathrm{d}}=1.44\right)$ mixture of water-DMSO $(25: 75$ by weight). $1 \mathrm{mM}$ of $\mathrm{LiCl}$ was added to screen electrostatic interactions with a Debye length $\kappa^{-1} \sim 7 \mathrm{~nm}$. Six volume fractions $(\varphi)$, ranging from 0.21 to maximum achievable packing $\varphi_{\max }(\geq 0.60)$, were prepared from a stock suspension in water-DMSO. This suspension was mixed with the probe particles directly inside custom-made cuvettes for CSLM. After centrifugation, the sediment was redispersed into the calculated amount of solvent needed to achieve the target volume fraction of host particles (using measured mass densities for the particles and assuming no excess mixing volume). In the case of $\varphi_{\max }$ the sample was exposed to high-speed centrifugation for several hours ( 1880 g, for 3.5 hours), and no redispersion was done. Here we define $\varphi_{\max }$ as the (achievable) upper glassy limit, where the host particles are tightly packed (close to random close packing $\left.\varphi_{\mathrm{rcp}}\right)$. Considering the size ratio of the host particles (1.17) and a possible expansion of the sediment after the centrifugation, the exact volume fraction of this densest sample should lie between 0.58 and $0.645\left(\approx \varphi_{\text {rсp }}\right)[14,15]$.

Reference case: smooth probes in a host system

Smooth probes were dispersed in the same host fluid and prepared in the same way as the rough probes in the $\lambda=1$ case. Four different volume fractions $(\varphi)$, ranging from 0.40 to the maximum packing $\varphi_{\max }$ (achievable upper glassy limit, as defined above) were prepared.

Raspberry probes in smaller sized hosts, $\lambda=4$

In this case, the host system consisted of smooth, polydisperse $\mathrm{SiO}_{2}$ particles $\left(\mathrm{r}_{\text {host- }}\right.$ mean $=25 \mathrm{~nm}$ with $20 \%$ polydispersity, the size distribution measured by AFM can be found in the Appendix). Four different volume fractions were prepared, ranging from 0.43 to maximum achievable packing ( $\varphi_{\max }$ upper glassy limit). Similar to our definition above, considering the possible expansion of the sediment after centrifugation and the polydispersity, here $\varphi_{\max }$ should lie between $0.58-0.68$, where the latter defines the $\varphi_{\text {rcp }}$ for polydisperse systems $[14,16]$. In 
each system, less than $0.5 \%$ of the sample volume consisted of rough raspberrylike probes with RITC labeled tracer berries in the shell.

Due to the small size of the host particles, centrifugation was not used for reaching high volume fractions. A stock suspension containing the host particles $(\varphi \approx 0.05)$ and raspberries (volume fraction $\left.\approx 210^{-4}\right)$ in water was subjected to solvent evaporation at $70-95^{\circ} \mathrm{C}$ under stirring and monitoring the weight. After the volume fraction reached $\approx 0.4$, a calculated amount of DMSO with dissolved $\mathrm{LiCl}$ was added under stirring. The sample was then subjected to a second evaporation stage. The endpoint of this stage corresponded to a target $\varphi$ of 0.52 (checked via weight), under the assumption that only water (having $\approx 25$ times higher vapor pressure than $\mathrm{DMSO}$ ) had evaporated. The target $\mathrm{LiCl}$ concentration in this sample was $1 \mathrm{mM}$.

During this step, some skin formation was observed, i.e., a thin layer of dry material on top of the fluid. Potential adverse effects hereof on the colloidal stability were mitigated by alternating between i) heating and ii) intensive stirring without heating. This was successful in removing visible particle aggregates. Using the finally obtained sample at $\varphi \approx 0.52$, two lower target concentrations $(\varphi=0.50,0.43)$ were reached by adding a known amount of solvent. The suspension at $\varphi_{\max }$ was obtained by centrifuging $(2000 \mathrm{~g}, 5.5 \mathrm{hr})$ a similarly prepared system with target $\varphi=0.50$ and not redispersing the sediment.

\subsubsection{Confocal Microscopy Experiments and Data Analysis}

Samples were homogenized via stirring before measurements of each $\varphi$, except the $\varphi_{\max }$ ones, where the measurements were performed right after centrifugation. Imaging was done using a confocal scanning laser microscope (CSLM) with a VisiTech 'VT-infinity' scan unit, a $488 \mathrm{~nm}$ and $561 \mathrm{~nm}$ laser, and 100x objective (1.49 N.A., oil immersion) connected to a Nikon Eclipse inverted microscope. 3D time series of $\sim 61 \times 60 \times 8-17 \mu \mathrm{m}^{3}$ volumes were captured at fixed time intervals, chosen between 4.0 and $90 \mathrm{~s}$. The lowest focal plane is set at least $10 \mu \mathrm{m}$ above the glass bottom to avoid wall effects on the particle dynamics.

3D localization of fluorescent berries was done using publicly available routines [17-19]. We used the method described in previous chapters to extract and separate the probe particles' rotations and translations in terms of mean squared displacements, MS(A)Ds, [11, 20]. 


\subsubsection{Estimation of noise levels for smooth probes}

The noise floors for the translations and rotations of the smooth probes were estimated by tracking the 3D locations of smooth probes immobilized on the glass cover slides and analyzing their MS(A)Ds. Due to the fluorescent tracer's size being smaller than the resolution limit of the CSLM, the signal from the fluorescent PS berries showed pixel biasing and higher noise levels for the translation and rotations of the smooth probes. The measured noise floors are added as dashed lines to the MS(A)D plots and the MS(A)Ds are presented in the Appendix.

\subsection{Results}

Figure 5.3.a-c shows translational and rotational dynamics of raspberry probes for the $\lambda=1$ and $\lambda=4$ cases. For the rough probes dispersed in asperity-sized hosts $(\lambda=1)$, the MSDs, $\left\langle\Delta \mathrm{r}^{2}(\tau)\right\rangle$, preserve their diffusive signature till $\varphi=0.42$ (see Figure 5.3.b). After this point, for $\varphi \geq 0.50$, the translational motion starts to show subdiffusive behavior. A plateau in $\left\langle\Delta \mathrm{r}^{2}\right\rangle$ arises at $\varphi=0.56$, showing that the particles become dynamically caged by their neighbors. Further increasing $\varphi$ to the point of maximum packing, the $\left\langle\Delta \mathrm{r}^{2}\right\rangle$ plateau amplitude decreases further, indicating that the cages become tighter. The MSADs, $\left\langle\Delta \theta^{2}(\tau)\right\rangle$, of this system, follow a qualitatively similar trend with host particle volume fraction. However, now the diffusive signature is preserved till $\varphi=0.52$ (see Figure 5.3.c). On further increasing the volume fraction, $\left\langle\Delta \theta^{2}\right\rangle$ develops a plateau, indicating that the rough probes' rotational motion becomes dynamically arrested via the surrounding host particles.

This 'rotational freezing' at high volume fractions of smooth host particles has not been found in earlier studies (to the best of our knowledge). It suggests that the surrounding host particles can tightly fill the vacant positions between the asperities and largely become immobilized, thereby causing a confinement effect on the rotational degree of freedom. This geometric hindrance also shows remarkable similarities with our previous work (in Chapter 4), in which raspberries' rotational degree of freedom was hindered due to interlocking configurations between the asperities of surrounding neighbors.

Figure 5.3.e-f shows the translational and rotational dynamics of the same rough probes but now dispersed in smaller-sized hosts $(\lambda=4)$. The translational motions 
indicated by the MSDs (see Figure 5.3.e) show a diffusive signature up to $\varphi=0.52$, which is a higher volume fraction than found for the $\lambda=1$ case. The prominent plateau behavior in $\left\langle\Delta r^{2}\right\rangle$ is visible only at the maximum packing fraction, i.e., at the upper glassy limit. Some cross-over of the MSDs occurs between the curves at $\varphi=0.52$ and $\varphi_{\max }$, at small lagtimes. It is not entirely clear what this means. It is beyond doubt that at $\varphi=0.52$, the probe raspberries are diffusing, while at $\varphi_{\max }(\approx 0.64)$, they are rattling in a cage. However, in dense systems of smooth, hard spheres, the rattling amplitudes have always been found to be smaller than the diffusive amplitudes [21]. It can also not be rigorously excluded that the proximity of the $\left\langle\Delta \mathrm{r}^{2}\right\rangle$ noise floor plays a role.

a)

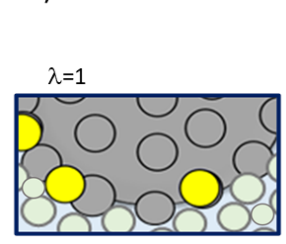

d)

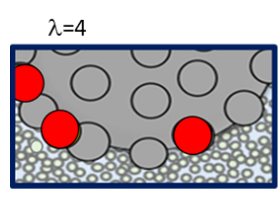

b)

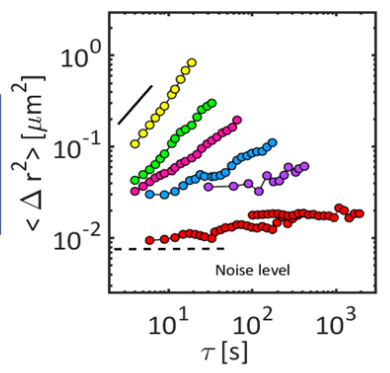

e)

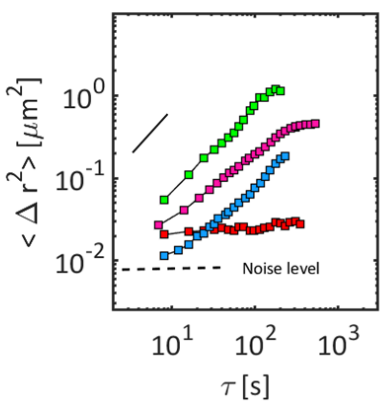

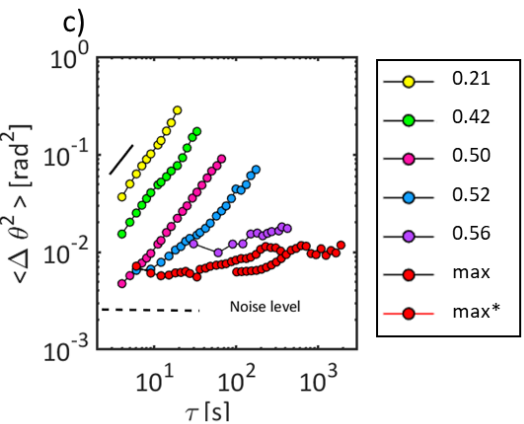

f)

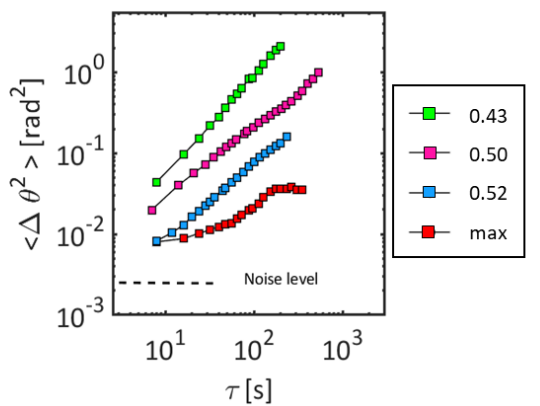

Figure 5.3. a) Schematic illustration of $\lambda=1$ case, b) Corresponding volume fraction $(\varphi)$ and lagtime $(\tau)$ dependent $<\Delta \mathrm{r}^{2}>$ and $\left.\left.\mathrm{c}\right)<\Delta \theta^{2}\right\rangle(*$ denotes different set of experiment with same $\varphi$, but with a longer $\tau$ and duration), d) Schematic illustration of $\lambda=4$ case, e) Corresponding volume fraction $(\varphi)$ and lagtime $(\tau)$ dependent $<\Delta \mathrm{r}^{2}>$ and $\mathrm{f}$ ) $<\Delta \theta^{2}>$. Black lines represent slope $=1$.

The rotational motion for the $\lambda=4$ case shows even stronger differences with the $\lambda=1$ case. As the host volume fraction is increased, the rotational motion shows 
a transition from diffusive to the subdiffusive regime. Even at the maximum packing fraction (the upper glassy limit for HS systems), the rotations of the raspberry probes do not get frozen (See Figure 5.3.f). It thus appears that, despite the glassy nature of the HS background fluid, and the even better ability of the small spheres at $\lambda=4$ to fill up the vacant spaces between the asperities of the raspberry probes, the raspberry rotations remain unconfined.

To further elucidate surface topography's effect in slowing down translations and rotations, we benchmark our rough probe experiments with a system containing smooth probes dispersed in the same host system as the rough probes at $\lambda=1$. The dependence of the MSD on host-particle volume fraction (see Figure. 5.4.b) shows a diffusive signature up to $\varphi=0.50$, while plateaus in $\left\langle\Delta \mathrm{r}^{2}(\tau)\right\rangle$ are observed for $\varphi \geq 0.57$. The two highest volume fractions appear to show a cage-breaking transition at larger lag times.

The rotational motions (shown in Figure 5.4.c) show a different volume fraction dependence. Starting from $\varphi=0.40$ till maximum packing, the MSAD stays in the diffusive regime with a reduction only in amplitude. This clear difference with the case of the rough probes is not unexpected. Because of the relatively smallsized asperities $\left(\mathrm{R}_{\max }=10-15 \mathrm{~nm}\right)$ on the 'smooth' probes, direct interlocking with the surrounding hosts ( $\mathrm{r}_{\text {host }}=100-120 \mathrm{~nm}$ ) is unlikely. Without such steric confinement in rotations, the smooth probes do not show arrested rotations.

This behavior of our 'smooth' reference system also shows similarities with the dynamics of smooth colloidal suspensions in literature [6, 22]. For a broad range of volume fractions, including liquid-to-crystal phase transitions, the rotational thermal motion was reported to remain diffusive with an $\left\langle\Delta \theta^{2}\right\rangle$ amplitude decrease by only a factor of 2.5 [22]. Meanwhile, the translational motion showed a qualitatively similar dependence as the one displayed in Figure 5.4.b. Our results corroborate the reasoning that for smooth, hard colloids near the glass transition and in dense crystalline phases, the rotational dynamics are dominated by hydrodynamic inter-actions, while the translations are strongly affected by both hydrodynamic and steric interactions [6, 22, 23]. 
a)

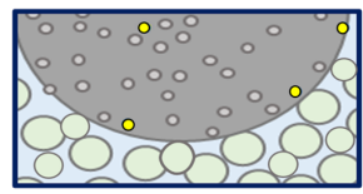

b)

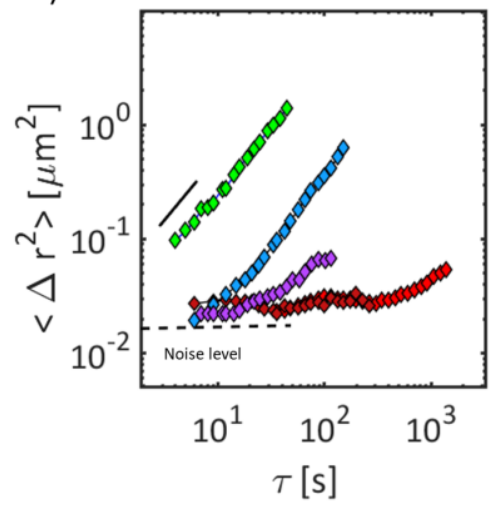

c)

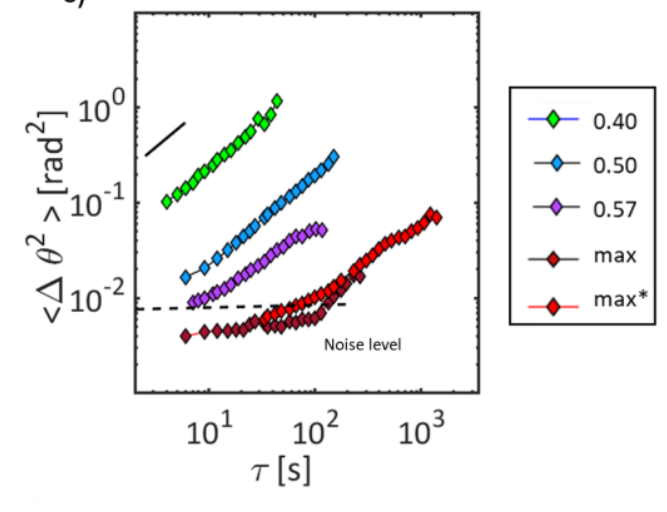

Figure 5.4. a) Schematic illustration of smooth reference case, b) Corresponding volume fraction $(\varphi)$ and lagtime $(\tau)$ dependent $\left\langle\Delta \mathrm{r}^{2}\right\rangle$ and $\left.\mathrm{c}\right)\left\langle\Delta \theta^{2}\right\rangle(*$ denotes the same $\varphi$ experiment with a longer $\tau$ and duration.

\subsubsection{Comparison of Diffusive Signatures}

To highlight and further inspect the differences between the three systems, we compare the powerlaw exponents $\alpha$, of $\left\langle\Delta \mathrm{r}^{2}(\tau)\right\rangle$ and $\left\langle\Delta \theta^{2}(\tau)\right\rangle$ as a function of the host particle volume fraction. First, we compare the exponents $\alpha_{t}$ and $\alpha_{r}$ per system: see Figures 5.5.a-c. It now becomes evident that the rough probes at $\lambda=1$ (Figure. 5.5.a) not only reaches a glassy state in both translation and rotation but also that there is an intermediate $\varphi$-range (around $\varphi=0.5$ ) in which the translation already becomes strongly subdiffusive while the rotational motion is still diffusive. A similar intermediate regime, indicative of different glass transition points for the two types of probe-motion was earlier found by us for all-raspberry dispersions. If the size of the smooth host particles gets reduced $(\lambda=4)$, this separation of 'glass transition points' disappears (Figure. 5.5.b). The system of 'smooth probes' in a background fluid of smaller smooth hosts only shows a translational glass transition (Figure. 5.5.c). 
Figure 5.5.d-e presents the same data in a different way. The comparison in Figure. 5.5.d. shows that all three systems show a translational glass transition. This is not unexpected, considering that translational motion in dense suspensions is dominated by the excluded volume of the particles. However, finer details like surface roughness can still matter. In this respect, it is noteworthy that the transitions in Figure. 5.5.d do not all occur at the same volume fraction.

The rotational slowdowns of the three systems (shown in Fig. 5.5.e) do not differ much up to $\varphi=0.5$, indicating that even the rotations of probe particles with significant roughness do not show a substantial alteration up to this point. For $\varphi>0.5$, the host particles do not only get 'pushed into' the asperities of the guest particles, but also the resistance of the host particles against collective rearrangements (as would be required by rotations of the guest particle) increases. The comparison of the three systems at the highest $\varphi$ shows dramatic differences in this respect. The $\lambda=1$ system shows rotational arrest $\left(\alpha_{r} 0\right)$, but for the $\lambda=4$ system, the 'glassifying' effect has already diminished. For the smooth probes, the effect is even less. Thus, we hypothesize that the rotational motion of a rough probe particle can only be frozen when the asperities are confined with a similarsized surrounding. 
a)

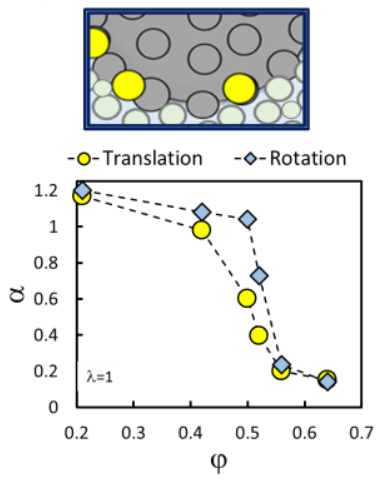

b)

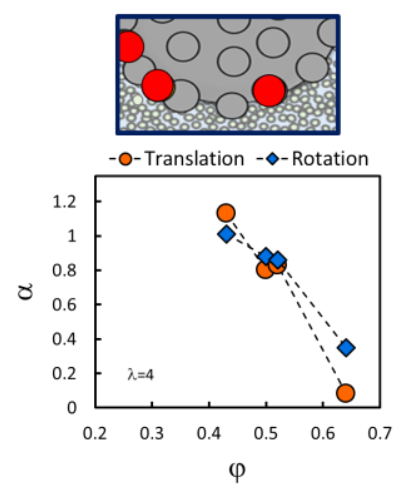

c)
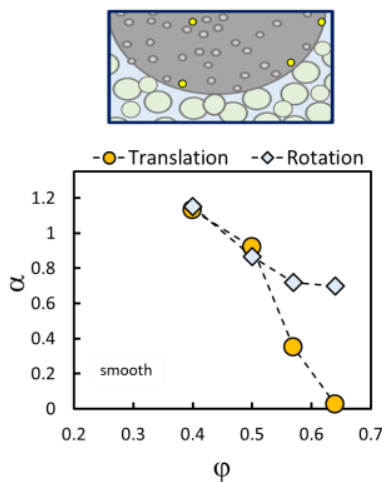
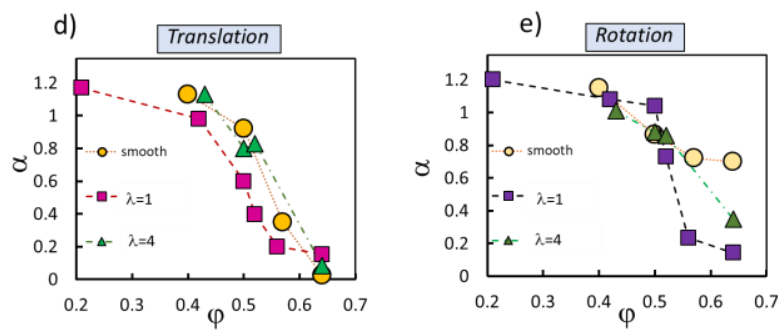

Figure 5.5. Log-log slope of the ensemble-averaged $<\Delta \mathrm{r}^{2}>$ and $\left.<\Delta \theta^{2}\right\rangle$ versus lagtime for different $\varphi$. Slopes are obtained from linear fits using lag times between 3 to $58 \mathrm{~s}$. a) $\lambda=1$, b) $\lambda=4$, c) Smooth case, $d-e)$ Comparison of the slowdown in 3 different cases plotted as $\log$-log slopes for d)translations and e) for rotations

\subsection{Discussion}

Our presented results highlight that confinement of the rotational degree of freedom for rough particles is not limited to like-body (i.e., raspberry to raspberry) interactions. The confinement of the rough probes' rotation requires a geometric frustration. As can be seen from our results for the $\lambda=1$ case, rotational motion can also be arrested when the vacant spaces between the asperities are filled with 'asperity sized' host particles. In both cases, the rough probes can experience similar spatial constraints. To test this hypothesis in terms of length scales, we reduced the size of the host particles. Our findings for the $\lambda=4$ case indicate that only filling up the asperities' interstitial spaces is not enough to arrest the rotational motion. While the results obtained here do not permit finding the underlying cause of this behavior, several hypotheses can be made. 
For the $\lambda=4$ case, the host particles' dynamics and relaxation time scales should be shorter than that of $\lambda=1$ case (at the same volume fraction) due to their smaller size. Although cage-breaking times could not be measured for either host system, the MSD amplitude of the guest particles is somewhat higher for the $\lambda=4$ case, suggesting less stiff confinement by the smaller host particles. Also, the rotations would be sensitive to such less stiff confinement: spaces that are created for translation also offer spaces for rotation. In our previous work (Chapter 4), we identified that on increasing the volume fractions, i.e., approaching the glass transition, rough particles' translation and rotation become correlated.

A somewhat related aspect concerns deviations from ideal Hard Sphere systems. Silica spheres in polar solvents generally carry charges on their surfaces, causing electrostatic repulsions. In our case, the Debye length, maintained at $7 \mathrm{~nm}$ for all three systems, is small compared to the size of the probe particles but not compared to the smallest host particles $(\lambda=4)$. The possible role of electrostatic interactions in our case is not easy to assess, and it could depend on the volume fraction of the host particles. It cannot be excluded that the 'less stiff confinement' mentioned above has a contribution from electrostatics.

When considering the dynamics of the host particles, it should also not be disregarded that the presence of a large probe can slow down the dynamics of nearby hosts. Simulation studies have shown that the presence of a large rough probe can significantly alter the probe bearing regions' host dynamics and introduce heterogeneity in supercooled liquids $[24,25]$. A similar analogy can be drawn regarding the confinement effect of the asperities to the filler host particles. Studies have shown that the dynamics of dense HS suspensions under confinement is slower than bulk, near the confining surfaces [21, 26, 27].

In that sense, we may hypothesize that the host particles between the asperities could behave like 'imprisoned fillers' and effectively become part of the raspberry probe. If this holds, smaller-sized hosts can have a 'smoothening' effect on the topography, resulting in a slightly bigger and smoother virtual probe. While the same confinement and following slowing down effect in the asperity filling hosts for $\lambda=1$ case would be present as well, these bigger fillers would not virtually smoothen the surface of the raspberry. Based on this, observed rotational dynamics would then be caused by diminished steric hindrance in the $\lambda=4$ case. 


\subsection{Chapter Outlook}

Given the results obtained here, we can propose several natural extensions of this work. In future experiments, simultaneously tracking the dynamics of the host particles for all cases and making comparisons between different host size systems can shed light on the dynamics of the host particles' and the differences in terms of confinement stiffness with respect to sizes. In addition, further diminishing the host particle size $(\lambda>>4)$ could reveal transitions with respect to asperity/host size when the effect of geometric confinement is lessened to the extremity.

In line with our previous proposal, simultaneous tracking of the host particle dynamics should reveal the effect of probes on the dynamics of the probe bearing and distant host particles. Designing such experiments and exploring different host sizes can shed light on the validity of the smoothening hypothesis and reveal if (and when) virtual near-complete smoothening can be achieved. It should also be noted here that all possible outlook proposals would require more frequent volume fraction data points and improved experimental quality for the densification and concentration determination, especially at maximum packing fractions.

Finally, we can comment on the possible extensions of our experimental system. Here, the experimental time window is limited to short time diffusion, and the number of volume fractions is partial. Experiments focusing on the long-time diffusion of the systems can show stimulating results regarding the validity of Stokes-Einstein-Debye relations and coupling/decoupling of the translations and rotations for rough probes. In addition, by making a direct analogy between probe-host dynamics and bio-molecule-host dynamics, our results can pave the way for obtaining insights into the diffusion of macromolecules in crowding environments where the coupling and decoupling of molecules are shape and interaction specific (e.g., in biochemical reactions, transport of solutes, protein processing) $[28,29]$. 


\section{Bibliography}

[1] Y.M. Joshi, Dynamics of colloidal glasses and gels, Annual review of chemical and biomolecular engineering, 5 (2014) 181-202.

[2] L. Cipelletti, E.R. Weeks, Glassy dynamics and dynamical heterogeneity in colloids, Dynamical heterogeneities in glasses, colloids, and granular media, 150 (2011) 110.

[3] P.G. Debenedetti, F.H. Stillinger, Supercooled liquids and the glass transition, Nature, 410 (2001) 259-267.

[4] S. Vivek, E.R. Weeks, Decoupling of translational and rotational diffusion in quasi-2D colloidal fluids, The Journal of chemical physics, 147 (2017) 134502.

[5] B. Ilhan, F. Mugele, M.H. Duits, Roughness Induced Rotational Slowdown near the Colloidal Glass Transition, Journal of Colloid and Interface Science, (2021).

[6] M. Kim, S.M. Anthony, S.C. Bae, S. Granick, Colloidal rotation near the colloidal glass transition, The Journal of chemical physics, 135 (2011) 054905.

[7] Y. Peng, L. Lai, Y.-S. Tai, K. Zhang, X. Xu, X. Cheng, Diffusion of ellipsoids in bacterial suspensions, Physical review letters, 116 (2016) 068303.

[8] Z. Zheng, F. Wang, Y. Han, Glass transitions in quasi-two-dimensional suspensions of colloidal ellipsoids, Physical review letters, 107 (2011) 065702.

[9] J. Roller, A. Laganapan, J.-M. Meijer, M. Fuchs, A. Zumbusch, Observation of liquid glass in suspensions of ellipsoidal colloids, Proceedings of the National Academy of Sciences, 118 (2021).

[10] W. Stöber, A. Fink, E. Bohn, Controlled growth of monodisperse silica spheres in the micron size range, Journal of colloid and interface science, 26 (1968) 62-69.

[11] B. Ilhan, J.J. Schoppink, F. Mugele, M.H.G. Duits, Spherical probes for simultaneous measurement of rotational and translational diffusion in 3 dimensions, Journal of Colloid and Interface Science, 576 (2020) 322-329. 
[12] A. Van Blaaderen, J. Van Geest, A. Vrij, Monodisperse colloidal silica spheres from tetraalkoxysilanes: particle formation and growth mechanism, Journal of Colioid and Interface Science, 154 (1992) 481-501.

[13] B. Ilhan, C. Annink, D. Nguyen, F. Mugele, I. Sîretanu, M.H. Duits, A method for reversible control over nano-roughness of colloidal particles, Colloids and surfaces A: Physicochemical and engineering aspects, 560 (2019) 50-58.

[14] R.S. Farr, R.D. Groot, Close packing density of polydisperse hard spheres, The Journal of chemical physics, 131 (2009) 244104.

[15] A.R. Kansal, S. Torquato, F.H. Stillinger, Computer generation of dense polydisperse sphere packings, The Journal of chemical physics, 117 (2002) 82128218.

[16] M. Hermes, M. Dijkstra, Jamming of polydisperse hard spheres: The effect of kinetic arrest, EPL (Europhysics Letters), 89 (2010) 38005.

[17] J.C. Crocker, E.R. Weeks, Particle tracking using IDL, Retreived from http://www. physics. emory. edu/faculty/weeks//idl, (2011).

[18] Y. Gao, M.L. Kilfoi, Accurate detection and complete tracking of large populations of features in three dimensions, Optics express, 17 (2009) 4685-4704.

[19] K.E. Jensen, N. Nakamura, Note: An iterative algorithm to improve colloidal particle locating, Review of Scientific Instruments, 87 (2016) 066103.

[20] G.L. Hunter, K.V. Edmond, M.T. Elsesser, E.R. Weeks, Tracking rotational diffusion of colloidal clusters, Optics express, 19 (2011) 17189-17202.

[21] H. Eral, D. van den Ende, F. Mugele, M. Duits, Influence of confinement by smooth and rough walls on particle dynamics in dense hard-sphere suspensions, Physical Review E, 80 (2009) 061403.

[22] S. Schütter, J. Roller, A. Kick, J.-M. Meijer, A. Zumbusch, Real-space imaging of translational and rotational dynamics of hard spheres from the fluid to the crystal, Soft matter, 13 (2017) 8240-8249.

[23] E.R. Weeks, Introduction to the Colloidal Glass Transition, Acs Macro Letters, 6 (2017) 27-34. 
[24] S.A. Mackowiak, J.M. Noble, L.J. Kaufman, Manifestations of probe presence on probe dynamics in supercooled liquids, The Journal of chemical physics, 135 (2011) 214503.

[25] R. Zangi, S.A. Mackowiak, L.J. Kaufman, Probe particles alter dynamic heterogeneities in simple supercooled systems, The Journal of chemical physics, 126 (2007) 104501.

[26] K.V. Edmond, C.R. Nugent, E.R. Weeks, Influence of confinement on dynamical heterogeneities in dense colloidal samples, Physical Review E, 85 (2012) 041401.

[27] C.R. Nugent, K.V. Edmond, H.N. Patel, E.R. Weeks, Colloidal glass transition observed in confinement, Physical Review Letters, 99 (2007).

[28] M. Roos, M. Ott, M. Hofmann, S. Link, E. Rössler, J. Balbach, A. Krushelnitsky, K. Saalwächter, Coupling and decoupling of rotational and translational diffusion of proteins under crowding conditions, Journal of the American Chemical Society, 138 (2016) 10365-10372.

[29] S. Zorrilla, M.A. Hink, A.J. Visser, M.P. Lillo, Translational and rotational motions of proteins in a protein crowded environment, Biophysical chemistry, 125 (2007) 298-305. 


\section{Appendix}

\section{Size distribution of $\lambda=4$ host particles}

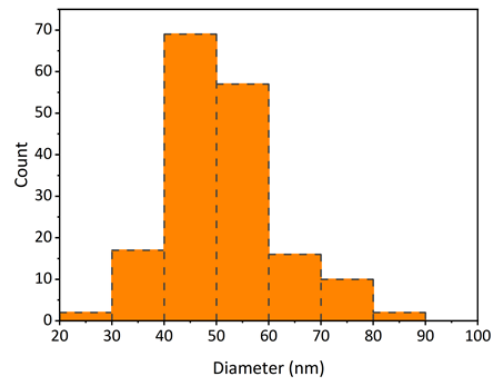

Figure A1. Size distribution of $\lambda=4$ hosts $\mathrm{SiO}_{2}$ particles (measured from AFM images)

\section{Noise floor measurements for smooth probes:}
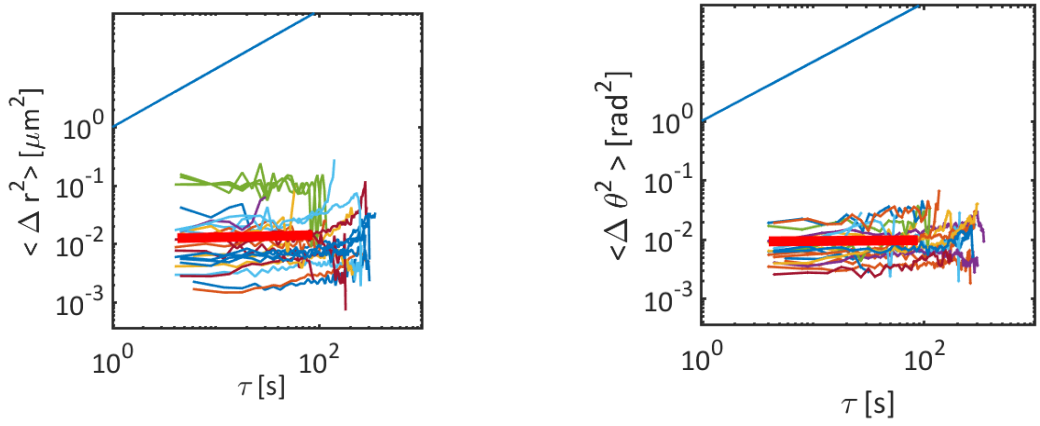

Figure A2. MSD (left) and MSAD (right) of smooth probes dried on a cover slip. Thick red lines are the mean. 



\section{Summary}

Topographical characteristics of colloidal particles can drastically alter the particles' thermal (or Brownian) motions in concentrated suspensions. While particle surface roughness is known to strongly influence various macroscopic behaviors, microscopic understanding of particle dynamics at high concentrations is lacking. Revealing the role of the surface requires a model colloidal system with explicit roughness characteristics. To disentangle the specific role of topography from other shape and geometry-dependent particle features, this thesis focuses exclusively on hard, globally spherical colloidal particles with well-defined roughness geometry. The dynamics are studied by accurately measuring the translational and rotational movements of the individual particles.

Chapter 2 addresses the challenge regarding the scarcity of the available methods for making well-defined rough colloids. A novel and relatively straightforward method to induce and control the nanoscale surface roughness on (water-based) polymer latex colloidal spheres is presented. The demonstrated method extends the methodology developed for flat polymer films. By exposing polymer latex particles of various sizes and surface groups to a degassed ionic solution, self-induced asperities (nanoscale) can be generated. The rough topography can be tailored via controlling the treatment temperature and the number of exposures. Due to the viscoelastic nature of the polymer colloids, applying mild thermal treatment for a particular duration results in smoothening (i.e., reversing the roughness) of the asperities on the surface. While this method contains its challenges in terms of upscaling the yield, it provides an opportunity to alter the topography in situ, which can be useful in studies and potential future applications, where on-demand changes in the topography are required without having to replace the colloidal fluid.

Revealing the role of roughness on the colloidal dynamics of dense suspensions requires a model colloidal system with well-defined and adjustable topography characteristics (e.g., bumps and dimples on a chosen length scale). Understanding the microscopic dynamics requires these model systems to be suitable for tracking translational and rotational motion simultaneously. Although measurement of translations under a (confocal) optical microscope is relatively 
simple, tracking rotations of spherical particles is certainly not trivial due to the absence of natural optical anisotropy and thus a natural frame of reference.

While the system presented in Chapter 2 can serve as a suitable model for rough colloids, introducing optical anisotropy into that colloidal system would require additional synthesis steps without opening up possibilities for optical experiments at high concentrations.

Chapter 3 addresses the scarcity of optically anisotropic, spherical probes with rough surfaces. This chapter presents a bottom-up synthesis route for rough spherical colloidal particles with optical anisotropy and the method to track their translations and rotations. The probe here is a raspberry-like all silica particle with fluorescent tracers in the shell. The synthesis method is upscaleable and provides a flexible design with uniform surface chemistry and tunability of the surface roughness. The applicability of the method is demonstrated by measuring the mean squared (angular) displacements of two types of probes, with different roughness amplitudes dispersed in two different media of different viscosities. The linearity of the MS(A)Ds, Gaussian displacement statistics, and isotropic diffusivity are all used to validate the methods described. These raspberry-like rotational probes serve as model systems to study the effect of roughness on colloidal dynamics in the following chapters.

Chapter 4 provides a unifying insight into dense colloidal suspensions' dynamics emerging due to a topography alteration to smooth spherical particles. The raspberry-like model rough colloidal system described in Chapter $\mathbf{3}$ is used to investigate the translational and rotational dynamics of the particles near the glass transition. Results show that roughness leads to a significantly lower maximum packing fraction, preceded by a broad concentration range in which the rotational Brownian motion changes signature from high-amplitude diffusive to lowamplitude 'rattling'. This substantial rotational slowdown occurs at significantly higher concentrations than for the translations, thus presenting a second glass transition. The drastic rotational slowdown occurs when the particle intersurface distance becomes comparable to the surface roughness amplitude. Mapping rotational dynamics onto a particle contact number provides the underlying physical picture that rotational slowdown is driven by geometric frustrations of the interacting surface asperities. The number of neighboring particles that can interact with a given particle via asperities affects the slowdown behavior. 
Chapter 5 builds on the proposed physical image described in Chapter 4, and aims to translate the insights into generic glass-forming colloidal systems.

To probe the "interlocking" of the asperities on rough particles with the individual smooth particles in dense suspensions, the raspberry particles are dispersed as a "guest" into different-sized host colloidal systems with smooth surfaces. By varying the asperity/host size ratio $(4,1)$, the effect and mechanism of confinement occurring on the asperities of the guests due to surrounding hosts are investigated. Variation of the host volume fraction identifies the concentration transitions in the dynamics of motions and slowing down of the guest particle. Again, both translation and rotational diffusion are taken into account.

The results show that the rotational slowdown due to geometric frustration of the surface asperities with the surrounding host system is the largest when the asperity/host ratio is comparable (1). In this case, with a gradual increase of the host volume fraction, the translational diffusion (of the guest particles) changes faster than the rotational diffusion. Particles undergo diffusive to low amplitude rattling rotational motion with increasing concentration. In order to benchmark the results of rough tracers, a case study where a new type of spherical probes with a smoothened surface (normal roughness amplitude $<1 \%$ radius) are dispersed in the same size host system. With the absence of asperities, these particles do not exhibit rotational arrest even at the highest concentrations, and the rotational Brownian motion shows a transition from diffusive to a mildly subdiffusive regime. 



\section{Outlook}

The research presented in this thesis can pave the way for future opportunities in answering fundamental questions and applications related to material design and engineering. Below are several potential future works and natural extensions of this work.

\section{Colloidal probes in passive and active microrheology}

The raspberry-like optical probes presented here are suitable candidates for investigating the microrheology of complex systems. Combined with optical microscopy techniques, the smoothened or rough colloids can be utilized as passive rotational microrheology probes to measure the shear response of viscoelastic, soft materials [1]. Additionally, our probes can be used as selfpropelled systems. They can be functionalized as active probes (e.g., replacing a desired portion of berries with Pt-based berries or utilizing the probe itself as Janus spheres). Such active particles can be embedded in samples and manipulated by applying known forces and displacements [2, 3]. In that respect, a future direction would be using them in active, probe-based rotational microrheology studies to measure the flow and deformation of complex systems. This can especially be essential in linking the microscopic properties to the macroscopic ones for many complex systems.

\section{The berry platform and material design}

The shape-dependent colloidal glass transition and the dynamical slowdown have already been explored to several extents [4-7], and the results obtained here offer the missing step in terms of topographical features' effect in glass transition. Our model rough colloidal system focuses on a (-near) spherical shape, but considering the design and synthesis flexibility, colloidal particles with complex shapes, varying aspect ratios and surface roughness can be synthesized using the berry platform. In this perspective, there are two avenues to follow for further mapping the coupling and decoupling of rotational and translational diffusion of such model colloidal systems in dense suspension. Here two of these are listed:

Systematically altering the spherical-shaped berries' size to an extent comparable to the (spherical) core particle's size would result in an extensive library of particles where shape, the length scale of steric interaction anisotropy and the 
roughness (on a larger scale) can be controlled in parallel. Such systems can be employed as model systems mimicking different molecules in glass transition studies. Controlling these parameters systematically and monitoring the coupling and decoupling in translational and rotational motions on single particle level would be a stepping stone in characterizing the relationship between shaperelated confinement anisotropy and dynamical slowdown near the glass transition.

Another extension would be answering the question: how can shape anisotropy combined with surface roughness affect the dynamics of colloidal systems? Studying the dynamical behavior of surface rough particles with anisotropic shapes (e.g., rough ellipsoidal particles where roughness is achieved by assembly of many smaller berries on the surface) can be very fruitful in terms of reveling hierarchical length scales governing the caging and steric confinement effects among the rough particles near the glass transition. Moreover, such particle systems can be promising for rich phase transition behavior.

\section{Engineering the flow behavior and local characterization}

Recent studies have shown that surface rough colloidal suspensions exhibit discontinuous shear thickening (DST) behavior at substantially lower volume fractions and shear rates $[8,9]$. This behavior has been attributed to increased interparticle friction among the rough particles [10]. Even though this behavior has been studied extensively in terms of bulk properties, the link between the microscopic properties and the macroscopic ones remains to be revealed. Given the results presented in Chapters 3 and 4, a possible extension would be direct imaging of model rough colloidal probes under shear or deformation at high volume fractions. Utilizing an experimental design with a shear cell and confocal microscopy or employing probe-driven active microrheology method, as described above, would help to understand constraints in sliding and rolling motions on the single-particle level. This could be integral in determining the consequences of such constraints regarding sliding friction $\left(\mu_{\mathrm{s}}\right)$ and rolling friction $\left(\mu_{\mathrm{r}}\right)$ and connecting the microscale to macroscale.

Moreover, within the perspective of material design, tailoring the surface roughness characteristics would raise the opportunity of engineering the DST response of rough systems. The particle loading required for DST response of rough particulate systems can be envisioned and tuned in many industrial applications such as shock-absorbing materials in ballistic protection systems [11] or processing of food industry products, such as chocolate [12]. 


\section{Bibliography}

[1] Z. Cheng, T. Mason, Rotational diffusion microrheology, Physical review letters, 90 (2003) 018304.

[2] L.G. Wilson, W.C. Poon, Small-world rheology: an introduction to probebased active microrheology, Physical Chemistry Chemical Physics, 13 (2011) 10617-10630.

[3] L. Wilson, A. Harrison, A.B. Schofield, J. Arlt, W. Poon, Passive and active microrheology of hard-sphere colloids, The Journal of Physical Chemistry B, 113 (2009) 3806-3812.

[4] G.L. Hunter, E.R. Weeks, The physics of the colloidal glass transition, Reports on Progress in Physics, 75 (2012).

[5] S. Vivek, E.R. Weeks, Decoupling of translational and rotational diffusion in quasi-2D colloidal fluids, The Journal of chemical physics, 147 (2017) 134502.

[6] J. Roller, A. Laganapan, J.-M. Meijer, M. Fuchs, A. Zumbusch, Observation of liquid glass in suspensions of ellipsoidal colloids, Proceedings of the National Academy of Sciences, 118 (2021).

[7] Z. Zheng, F. Wang, Y. Han, Glass transitions in quasi-two-dimensional suspensions of colloidal ellipsoids, Physical review letters, 107 (2011) 065702.

[8] C.P. Hsu, S.N. Ramakrishna, M. Zanini, N.D. Spencer, L. Isa, Roughnessdependent tribology effects on discontinuous shear thickening, Proceedings of the National Academy of Sciences of the United States of America, 115 (2018) 5117-5122.

[9] L.C. Hsiao, S. Jamali, E. Glynos, P.F. Green, R.G. Larson, M.J. Solomon, Rheological State Diagrams for Rough Colloids in Shear Flow, Physical Review Letters, 119 (2017).

[10] A. Singh, C. Ness, R. Seto, J.J. de Pablo, H.M. Jaeger, Shear Thickening and Jamming of Dense Suspensions: The "Roll" of Friction, Physical Review Letters, 124 (2020).

[11] M. Hasanzadeh, V. Mottaghitalab, The role of shear-thickening fluids (STFs) in ballistic and stab-resistance improvement of flexible armor, Journal of materials engineering and performance, 23 (2014) 1182-1196. 
[12] E. Blanco, D.J. Hodgson, M. Hermes, R. Besseling, G.L. Hunter, P.M. Chaikin, M.E. Cates, I. Van Damme, W.C. Poon, Conching chocolate is a prototypical transition from frictionally jammed solid to flowable suspension with maximal solid content, Proceedings of the National Academy of Sciences, 116 (2019) 10303-10308. 


\section{Samenvatting}

Topografische kenmerken van colloïdale deeltjes kunnen de thermische (of Brownse) bewegingen van de deeltjes in geconcentreerde suspensies drastisch veranderen. Hoewel bekend is dat de oppervlakteruwheid van deeltjes verschillende macroscopische gedragingen sterk kan beïnvloeden, ontbreekt ook nog veel microscopisch begrip van de deeltjes dynamica bij hoge concentraties. Het onthullen van de rol van het oppervlak vereist een colloïdaal modelsysteem met expliciete ruwheidskenmerken. Om de specifieke rol van topografie te ontwarren van andere vorm- en geometrie-afhankelijke deeltjeskenmerken, richt dit proefschrift zich uitsluitend op harde, globaal bolvormige colloïdale deeltjes met goed gedefinieerde ruwheidsgeometrie. De dynamica wordt bestudeerd door nauwkeurige meting van de translatie- en rotatie-bewegingen van de individuele deeltjes.

Hoofdstuk 2 gaat in op de uitdaging met betrekking tot de schaarste van de beschikbare methoden voor het maken van goed gedefinieerde ruwe colloïden. Een nieuwe en relatief eenvoudige methode om nanoschaal oppervlakteruwheid te induceren en controleren in watergedragen poly-meer latex bollen, wordt gepresenteerd. De methode behelst een uitbreiding van een methodologie die is ontwikkeld voor vlakke polymeer-films. Door polymeerlatexdeeltjes met verschillende groottes en oppervlaktegroepen bloot te stellen aan een ontgaste zoutoplossing, kunnen oneffenheden op de nanoschaal worden gegenereerd. De ruwe topografie kan worden aangepast via de behandelings-temperatuur en het aantal behandelingen. Vanwege de visco-elastische aard van de polymeercolloïden, resulteert het toepassen van een milde thermische behandeling in het gladstrijken (d.w.z. het omkeren van de ruwheid) van de oneffenheden op het oppervlak. Deze mogelijkheid om de topografie in situ te wijzigen kan nuttig zijn in studies en mogelijk toekomstige toepassingen, waar on-demand veranderingen in de topografie nodig zijn zonder de colloïdale vloeistof te hoeven vervangen.

Het onthullen van de invloed van ruwheid op de colloïdale dynamica van geconcentreerde suspensies vereist een colloïdaal modelsysteem met goed gedefinieerde en instelbare topografische kenmerken (bijv. uitstulpingen en indentaties op een gekozen lengteschaal). Om de microscopische dynamica te begrijpen, moeten deze modelsystemen geschikt zijn voor het gelijktijdig volgen van translatie- en rotatiebewegingen. Hoewel meting van translaties onder een 
(confocale) optische microscoop relatief eenvoudig is, is het volgen van rotaties van bolvormige deeltjes zeker niet triviaal vanwege de afwezigheid van natuurlijke optische anisotropie en daarmee een natuurlijk referentiekader. Hoewel het in Hoofdstuk 2 gepresenteerde systeem kan dienen als een geschikt model voor ruwe colloïden, zou het introduceren van optische anisotropie in dat colloïdale systeem extra synthese stappen vereisen, zonder mogelijkheden te scheppen voor optische experimenten bij hoge concentraties.

Hoofdstuk 3 behandelt de schaarste aan optisch anisotrope, bolvormige silica deeltjes met grote oppervlakteruwheid. Een bottom-up syntheseroute wordt gepresenteerd voor ruwe sferische colloïdale deeltjes met optische anisotropie. Ook wordt de methode besproken waarmee de translaties en rotaties gemeten kunnen worden. De deeltjes zijn nu framboos-achtig (een grote bol bedekt met vele kleine bollen) met fluorescerende tracers in de schil. De synthesemethode is opschaalbaar en zorgt voor een flexibel ontwerp met uniforme oppervlaktechemie en afstembaarheid van de oppervlakteruwheid. De toepasbaarheid van de methode wordt aangetoond door het meten van de thermische beweging in verdunde dispersies. De gemiddelde kwadratische (hoek) verplaatsingen van twee soorten deeltjes met verschillende ruwheidsamplitudes worden gemeten in media met verschillende viscositeit. De lineaire tijdsafhankelijkheid van deze ver-plaatsings functies, de Gaussiaanse verplaatsingsstatistieken en isotrope diffusiviteit worden allemaal gebruikt om de deeltjes te valideren als diffusieve probes. De framboos-achtige deeltjes worden gebruikt om het effect van ruwheid op de colloïdale dynamica te bestuderen in de volgende hoofdstukken.

Hoofdstuk 4 geeft een verenigend inzicht in de veranderingen die ontstaan in de dynamica van geconcentreerde colloïdale suspensies, als gevolg van een topografische verandering van de gladde bolvormige deeltjes opper-vlakken. Het framboos-achtige colloïdale systeem zoals beschreven in Hoofdstuk 3 wordt gebruikt om de translatie- en rotatiedynamica van de deeltjes nabij de colloïdale glasovergang te onderzoeken. Resultaten tonen aan dat de oppervlakteruwheid leidt tot een significant lagere maximale pakkingsfractie. In het glasachtige regime zien we een breed concentratie-bereik waarin de roterende Brownse beweging verandert van diffusief met hoge amplitude naar 'ratelend' met lage amplitude. Deze overgang treedt op bij aanzienlijk hogere concentraties dan die van de translaties, zodat gesproken kan worden van een tweede glas-overgang. De meest drastische vertraging van de rotatiebeweging treedt op wanneer de afstand tussen de deeltjesoppervlakken vergelijkbaar wordt met de 
oppervlakteruwheids-amplitude. Het aldus gezamenlijk in kaart brengen van de translatie- en rotatie-dynamica levert het onderliggende fysieke beeld op dat de rotatie-vertraging wordt veroorzaakt door geometrische frustratie: het op elkaar inhaken van de oppervlakte structuren van de interacterende deeltjes. Ook het aantal naburige deeltjes dat op deze manier kan interacteren met een gegeven deeltje speelt hierbij een rol.

Hoofdstuk 5 bouwt voort op het voorgestelde fysieke beeld zoals beschreven in Hoofdstuk 4, en beoogt de inzichten te vertalen naar generieke glas-vormende colloïdale systemen. Om het 'in elkaar grijpen' van de opper-vlakte oneffenheden op de ruwe deeltjes, met individuele gladde deeltjes in zeer geconcentreerde suspensies te onderzoeken, worden de framboos-deeltjes als 'gast' gedispergeerd in colloïdale systemen van gladde deeltjes van verschillende grootte. Door de verhouding tussen de afmetingen van de oppervlakte-oneffenheden en de diameter van de gladde deeltjes te variëren (tussen 1 en 4), wordt onderzocht hoe belangrijk het is dat de oppervlakte-structuren van de gast- en gastheer-deeltjes 'op elkaar passen'. Variatie van de gastheer volumefractie wordt gedaan om te onderzoeken hoe 'glasachtig' de suspensie moet zijn om het gastdeeltje effectief te kunnen immobiliseren. Wederom worden zowel de translatie als de rotatie diffusie hierbij in ogenschouw genomen.

De resultaten laten zien dat de vertraging van de rotatiebeweging als gevolg van geometrische frustratie van de oppervlakte-oneffenheden met het omringende gastheer systeem, het grootst is wanneer de ruwheidsamplitude van de gastdeeltjes en de afmeting van de gastheer deeltjes vergelijkbaar zijn. In dat geval zien we bij geleidelijke verhoging van de gastheer volumefractie, dat de translatie diffusie (van de gast deeltjes) sneller verandert dan de rotatie diffusie. De rotatie diffusie bereikt ook voor dit systeem een regime waar de deeltjes slechts in een beperkt hoekbereik heen en weer kunnen 'ratelen'. Soortgelijke experimenten met (nieuwe) framboos-deeltjes met een relatief glad oppervlak (ruwheidsamplitude ongeveer 1\% van de deeltjesgrootte) gedispergeerd in hetzelfde gastheer systeem laten zien dat de drastische verandering in de rotatie diffusie in dit geval uitblijft. 



\section{Publication list}

\section{Related to this thesis:}

Ilhan, B., Annink, C., Nguyen, D. V., Mugele, F., Sîretanu, I., \& Duits, M. H. (2019). A method for reversible control over nano-roughness of colloidal particles. Colloids and surfaces A: Physicochemical and engineering aspects, 560, 50-58.

Ilhan, B., Schoppink, J. J., Mugele, F., \& Duits, M. H. (2020). Spherical probes for simultaneous measurement of rotational and translational diffusion in 3 dimensions. Journal of colloid and interface science, 576, 322-329.

Ilhan, B., Mugele, F., \& Duits, M. H. (2022). Roughness induced rotational slowdown near the colloidal glass transition. Journal of colloid and interface science, 607, 1709-1716.

Ilhan, B., Mugele, F., \& Duits, M. H. (202x). Roughness as a Tool to Investigate Steric Interaction Length Scale near the Colloidal Glass Transition (In preparation)

\section{Other publications from the author:}

Ilhan, B., Kurt, M., \& Ertürk, H. (2016). Experimental investigation of heat transfer enhancement and viscosity change of hBN nanofluids. Experimental Thermal and Fluid Science, 77, 272-283.

Ilhan, B. \& Ertürk, H. (2017). Experimental characterization of laminar forced convection of hBN-water nanofluid in circular pipe. International Journal of Heat and Mass Transfer, 111, 500-507. 


\section{Acknowledgments}

This thesis marks the end of my Ph.D. research. It was an exciting and transformative journey, and I feel lucky to be surrounded by so many inspiring people whose contributions made this experience possible and fun.

First of all, I would like to thank my supervisors. Frieder, thank you for giving me the opportunity to do my Ph.D. at PCF. I greatly appreciate your support and guidance during my Ph.D. I enjoyed our discussions and learned a lot from you. Your refreshing questions and insights triggered several new ideas and pathways for me over the last four years. Michel, I am very thankful for your support and supervision. You introduced me to the fantastic world of silica colloids and taught me the essence of synthesis at the beginning of my Ph.D., which opened up many great possibilities for me to pursue in my research. I learned a lot from you and always enjoyed our discussions about science and life. Our brainstorming sessions were very valuable for me. They often provided me the boost of motivation that I needed from time to time. Stefan, thank you for introducing me to the world of simulations and giving me a chance to combine the experimental approach with the computational one. Our discontinuous shear thickening project allowed me to explore and research with a fresh perspective. I also thank you for many discussions and thoughtful questions about my research.

I would like to thank all my committee members for their interest in my research. I am grateful for their time in reading my thesis and providing valuable feedback.

Next, I would like to extend my gratitude to my friends and colleagues from PCF for helping this journey be fun and comfortable. Dirk, I sincerely appreciate your interest and insights into my research and thank you for many fruitful discussions and suggestions. I have always admired your approach to scientific problems and your ability to understand and simplify them for the listener. Igor, thank you for teaching me how to use AFM; I enjoyed working together with you during our bumpy paper. Isabel, thank you for your support and invaluable help during my time at PCF. Carla, thank you for making my first days in the lab so welcoming and easier.

Special thanks go to my Hao Wu. Hao, I cannot imagine how I could have gone through these four years without you. You celebrated my victories and happy moments as my cheerleader; you shared my frustrations and problems as if they 
were yours. We spent so many days and nights in the lab and the office together, and having you right by my side always encouraged and inspired me. Your friendship has been a home away from home for me. I feel extremely lucky and privileged to have you in my life.

Next in line are my paranymphs Saravana \& Sachin. Sara, you have been my half mechanical half aerospace engineer friend at a fundamental science lab; you listened to me and thoughtfully helped me whenever I needed a critical and fresh pair of eyes on my research. I thank you a lot for your friendship and great support. I enjoyed our encouraging life \& science $\&$ future discussions and $4 \mathrm{pm}$ yogurt sessions at the office. I am looking forward to the day we will share yogurt rice! Sachin (Sacu-e), my "tru." friend and my Ph.D. twin, we started Ph.D. journey almost together, and here we are ending it almost together. Is this a glitch or our final jinx? Your friendship and support made these four years truly manageable and enjoyable. Thank you for understanding me, even at times without me saying a word. Our daily and sometimes virtual fresh air breaks have been wonderful, and I greatly value our "high-tech human touch" discussions. Qierui (Cherry, Berry), thanks to our early morning coffee sessions in the office, I started my day energetic many times. I am thankful for your friendship, support and also for many delicious dinners at your home. I always enjoyed our long-lasting, balancing the universe meetings together with Sara. Duy, it was really fun to have our late-night and weekend conversations about life and image processing. I have always admired your unlimited energy, and I cannot wait to see your great next work. Özlem, thank you for your support and friendship over the last years, I greatly enjoyed our Turkish coffee chats and long walks around the campus, they were nice boosts of morale for me. Su, you always made me very happy with your sincere smile and positive attitude to life. I would also like to thank my student Jelle for his support in my research. To rest of my recent and former colleagues from PCF: Ranabir, Aditya, Jun, Nathalie, Alessandro, Diana, Amani, Amrutha, Harmen, Ashit, Amy, Olena, Aram, Davood, Arjen, Simone, Arvind, Chamy, Stelian, Frank, Daniel, Bas, Martin, Niels, Rudy, Vincent thank you for making my time at PCF memorable and pleasant!

I would also like to thank my friends outside of work for their wonderful support and friendship. Itır, thank you for being an amazing friend for long-lasting years. Even though life threw us to different continents for several years, I have never felt the distance; you have always been there for me. Pelin, thank you for your great friendship and support. You have always been there for me from the first weeks of my Ph.D. to the last. Whenever I needed help outside my lab, you were 
the first person I reached out to. Buse (Buzim), thank you for being with me all the time and making me always feel loved and supported through these years. Ece, Sinem, thank you for being one message or video call away whenever I would like to chat/vent. Elif, Cemre, Gülfem, Fatih, Yağız, Hao Shi, Nazlı, Harika, Bahar, thank you for your support and encouragement throughout all these years!

Onur, thank you for being you and taking this journey together with me. I am incredibly fortunate and glad I spent these four years with you by my side. With your endless love and support, I have felt everything is possible. Thank you for always encouraging me and helping me to overcome many obstacles of Ph.D. and life. And of course, thank you, my Patik, for being the best companion a person can dream of and sharing your life, love and happiness with me as always. Minik Kızım, you are always in my heart. Take care until we meet again in another life and time.

Last but least, I would like to thank my family, my mom Düriye, my dad Rifat and my brother Serav for their enourmous love, support and faith in me throughout my whole life. Anne, Baba hep arkamda olduğunuz ve beni her zaman destekledğiniz için ne kadar teşekkür etsem azdır. Sizin desteğiniz ve sevginiz bana her zaman güç verdi ve her sorunu aşabileceğime inanmamı sağladı. Sizi seviyorum!

Beybin İlhan

University of Twente

December 2021 



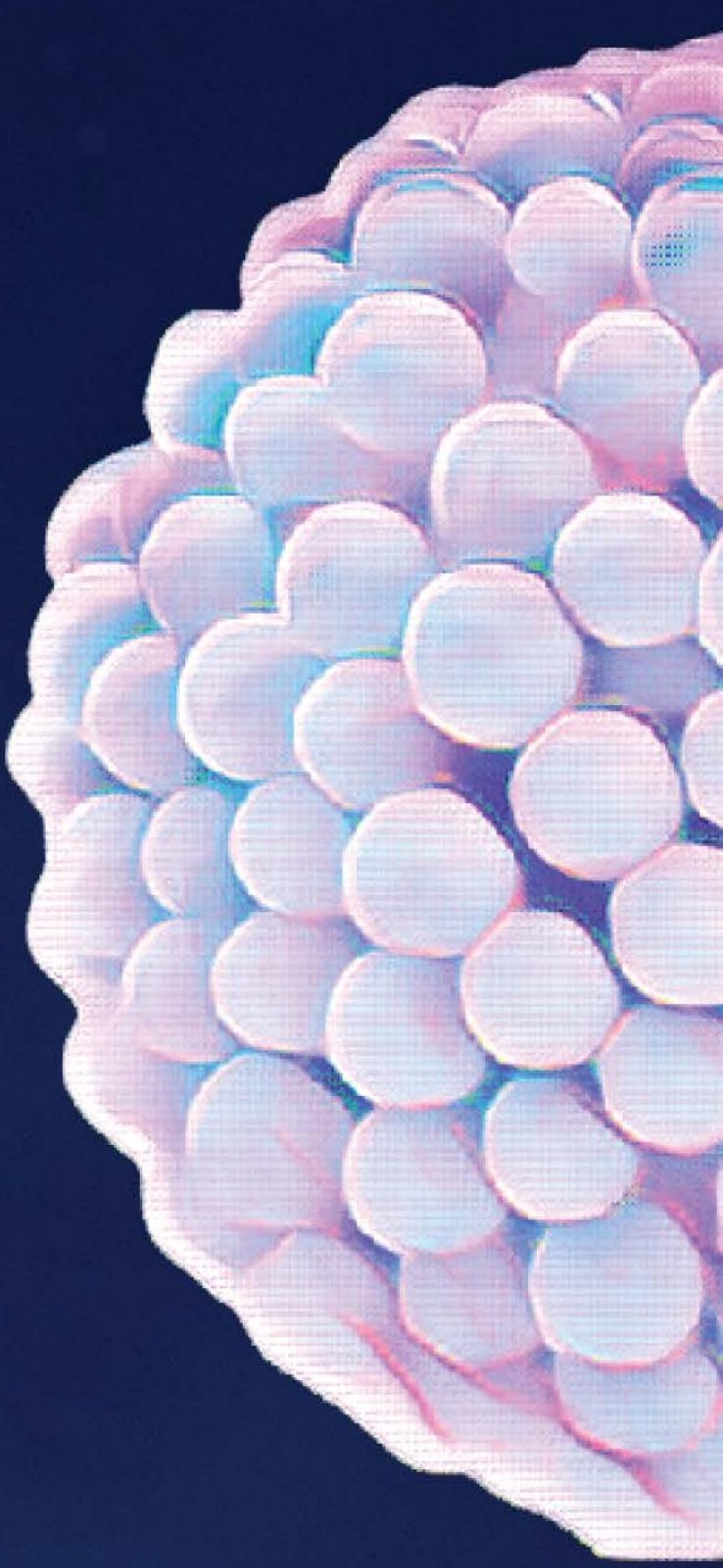

ISBN:978-90-365-5308-7 